Portland State University

PDXScholar

8-23-1996

\title{
Calibration of a CCD Camera and Correction of its Images
}

Armin Rest

Portland State University

Follow this and additional works at: https://pdxscholar.library.pdx.edu/open_access_etds

Part of the Physics Commons

Let us know how access to this document benefits you.

Recommended Citation

Rest, Armin, "Calibration of a CCD Camera and Correction of its Images" (1996). Dissertations and Theses. Paper 5186.

https://doi.org/10.15760/etd.7062

This Thesis is brought to you for free and open access. It has been accepted for inclusion in Dissertations and Theses by an authorized administrator of PDXScholar. Please contact us if we can make this document more accessible: pdxscholar@pdx.edu. 


\section{Thesis APPROVAL}

The abstract and thesis of Armin Rest for the Master of Science in Physics were presented August 23, 1996, and accepted by the thesis committee and the department.

Committee Approvals:
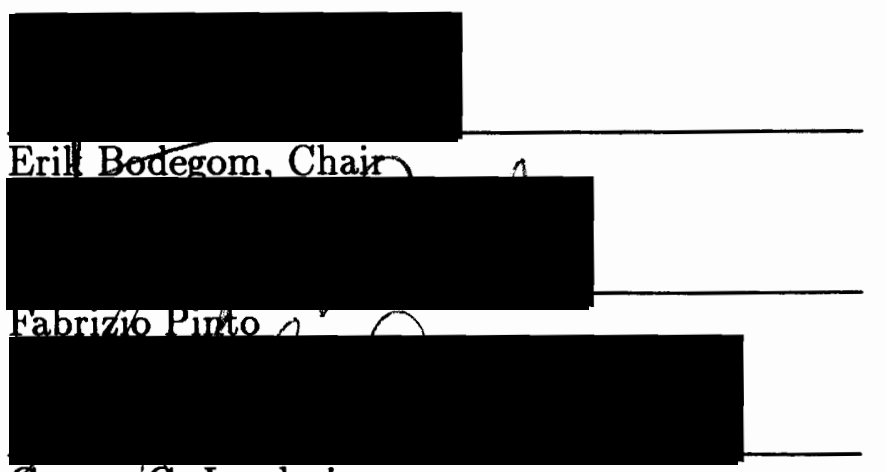

George G. Lendaris

Representative of the Office of Graduate Studies

Department APPRoval:

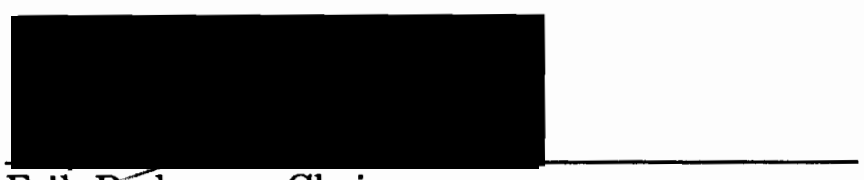

Erik Bodegom, Chair

Department of Physics

$* * * * * * * * * * * * * * * * * * * * * * * * * * * * * * * * * * * * * * * * * * * * * * * * * * * * * * * * * * * * * * * * * * * * *$

Accepted for Portland State University by the Library

by

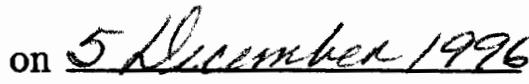




\begin{abstract}
The abstract of the thesis of Armin Rest for the Master of Science in Physics presented August 23, 1996.
\end{abstract}

Title: Calibration of a CCD camera and correction of its images

Charge-Coupled-Device (CCD) cameras have opened a new world in astronomy and other related sciences with their high quantum efficiency, stability, linearity, and easy handling. Nevertheless, there is still noise in raw CCD images and even more noise is added through the image calibration process. This makes it essential to know exactly how the calibration process impacts the noise level in the image. The properties and characteristics of the calibration frames were explored. This was done for bias frames, dark frames and flat-field frames at different temperatures and for different exposure times.

At first, it seemed advantageous to scale down a dark frame from a high temperature to the temperature at which the image is taken. However, the different pixel populations have different doubling temperatures. Although the main population could be scaled down accurately, the hot pixel populations could not. A global doubling temperature cannot be used to scale down dark frames taken at one temperature to calibrate the image taken at another temperature. 
It was discovered that the dark count increased if the chip was exposed to light prior to measurements of the dark count. This increase, denoted as dark offset, is dependent on the time and intensity of the prior exposure of the chip to light. The dark offset decayes with a characteristic time constant of 50 seconds. The cause might be due to storage effects within chip.

It was found that the standard procedures for image calibration did not always generate the best and fastest way to process an image with a high signal-to-noise ratio. This was shown for both master dark frames and master flat-field frames. In a real world example, possible night sessions using master frame calibration are explained. Three sessions are discussed in detail concerning the trade-offs in imaging time, memory requirements, calibration time, and noise level.

An efficient method for obtaining a noise map of an image was developed, i.e., a method for determining how accurate single pixel values are, by approximating the noise in several different cases. 


\title{
CALIBRATION OF A CCD CAMERA AND CORRECTION OF ITS IMAGES
}

\author{
By \\ ARMIN REST
}

A thesis submitted in partial fulfillment of the

requirements for the degree of

\author{
MASTER OF SCIENCE \\ in \\ PHYSICS
}

Portland State University

1996 
Für

Marlene 


\section{Acknowledgments}

I want to express my appreciation and gratitude to all the different people who have supported me throughout my research. I am indebted to my advisor Dr. Fabrizio Pinto for providing an interesting and challenging project and for all his guidance throughout the project. I am also grateful to Dr. Erik Bodegom and the Physics Department. Dr. Bodegom's unique humor, encouraging help and unforgetable ways of solving problems will always be remembered. I deeply appreciate the help of Dennis Luse; without his telescope I would not have been able to take any star images. I am grateful to Tom Misley for all the time and great effort he spent in enlightening my understanding of the English language. I am afraid the task was not easy. Very special thanks to my roommate and friend Thomas Herzinger for his patient help by solving all the problems I had related to computers. I will remember all the fun we had together in this year, all the way to the year 2008. I would also like to acknowledge the University of Portland and NASA for the support in acquiring of the camera. Lynn, Shawn, Tony, Sven, Thomas and a lot of other 
people made the time I spent in the Physics Computer Lab a time full of laughs and good memories. I want to thank my family and all my friends for the love and friendship they have given me. I have spent one of the best times of my life here in Portland. Thank you! 


\section{Contents}

$\begin{array}{lll}\text { Acknowledgments } & \text { ii }\end{array}$

$\begin{array}{ll}\text { Introduction } & \mathbf{1}\end{array}$

0.1 History of Light Detectors in Astronomy . . . . . . . . . . . . 1

0.2 Principles of a CCD Camera . . . . . . . . . . . 3

0.3 Comparison of CCD's and Other Light Detectors $\ldots \ldots \ldots$

I Theoretical Background: Noise and Repeatable Pat$\begin{array}{ll}\text { terns in CCD Images } & 8\end{array}$

1 Repeatable Patterns $\quad 9$

2 Noise in Images $\quad 13$

2.1 The Statistical Nature of Noise . . . . . . . . . . 13

2.2 The Sources of Noise . . . . . . . . . . . . . 15 
2.3 Signal-to-Noise Ratio, $\frac{S}{N} \ldots \ldots \ldots \ldots$

2.4 Image Arithmetic . . . . . . . . . . . . . . . 17

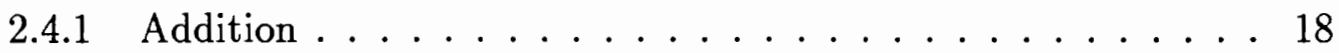

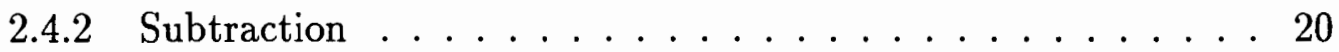

2.4.3 Multiplication ................. 20

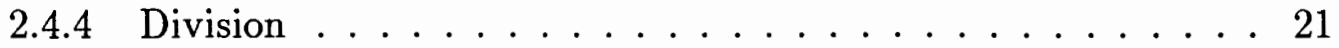

2.4.5 Multiplication with a Constant ............ 23

2.4.6 Combining Images . . . . . . . . . . . . . . . 23

2.5 Noise in the Calibrated Signal . . . . . . . . . . . . 26

II Calibration of CCD Images $\quad 30$

3 Instruments and Methods $\quad 32$

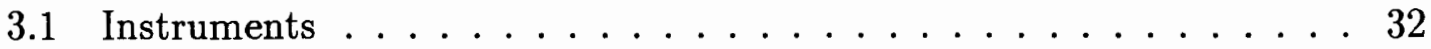

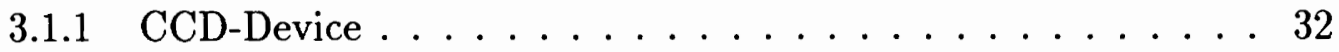

3.1.2 Mira Software .................. 34

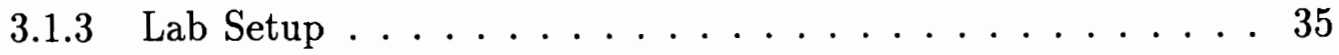

3.1.4 Telescope Setup . . . . . . . . . . . . . 35

3.2 Methods . . . . . . . . . . . . . . . 36

3.2 .1 Measuring the Noise of an Image $\ldots \ldots \ldots \ldots$

3.2.2 Experimental Procedures in the Lab . . . . . . . . . 39 


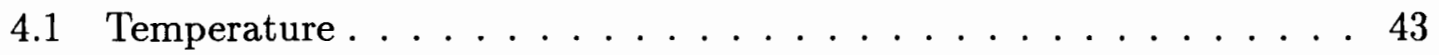

4.1.1 Bias Noise ................... 46

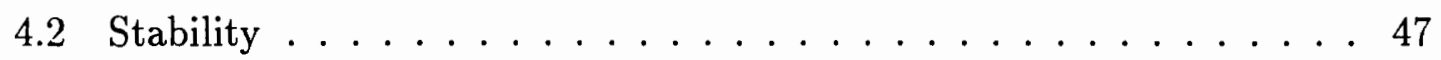

5 Dark Frames $\quad 48$

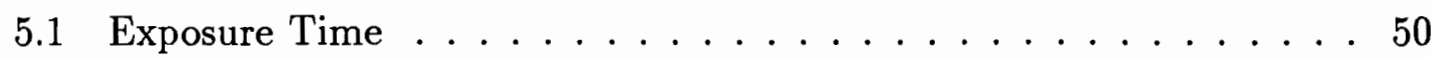

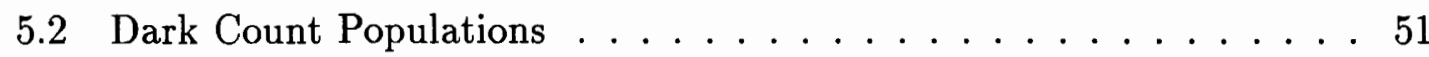

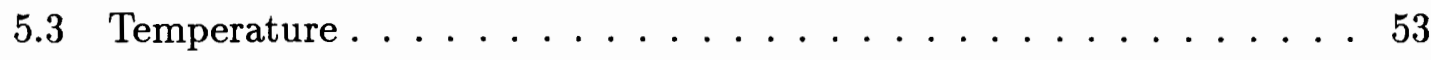

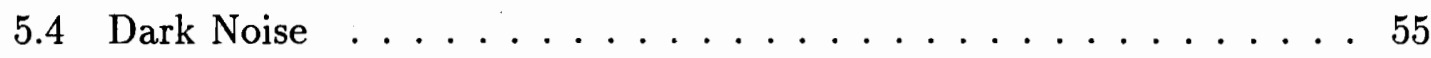

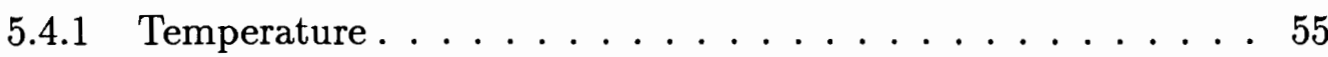

5.4 .2 Exposure Time .................... 56

5.5 Is it Possible to Use Dark Frames Taken at Another Temperature ? 57

5.6 Effect of Past Images on the Dark and Bias Count . . . . . . . . . 62

6 Flat-Field Frames $\quad 68$

6.1 When is it Necessary to Take a New Flat-Field Frame . . . . . . . 70

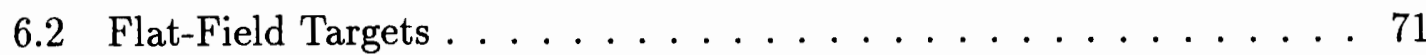

7 Master Frames $\quad \mathbf{7 4}$

7.1 Generating a Master Bias Frame.............. 75

7.2 Generating a Master Dark Frame . . . . . . . . . . . . 77 
7.3 Generating a Master Flat-Field Frame . . . . . . . . . . . . 80

8 Real World Example $\quad 85$

8.1 Comparison of the Three Night Sessions . . . . . . . . . . . 90

8.2 Quantitative Estimation of the Noise Levels . . . . . . . . . . 91

$\begin{array}{llr}9 & \text { Conclusions } & 98\end{array}$

$\begin{array}{ll}\text { Appendix } & 102\end{array}$

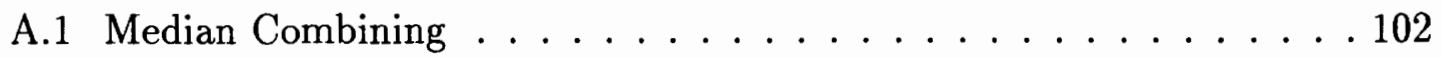

A.2 Normalization in Combining feature . . . . . . . . . 105

A.3 Minor Problems ..................... 109 


\section{List of Tables}

1 Overview of signal and noise levels in image arithmetic . . . . . . 24

2 Two ways to generate a master dark frame $\ldots \ldots \ldots \ldots$

3 Two ways to generate a master flat-field frame $\ldots \ldots \ldots . \ldots 8$

4 Three possibilities of how a night session might look . . . . . . 88

$5 \quad$ Noise levels of the night sessions . . . . . . . . . . . . 89 


\section{List of Figures}

1 Comparison of the quantum efficiency of CCD-device to other light detectors .................... 6

2 Ring Nebula M-57: calibrated and uncalibrated image . . . . . . . 28

3 Hercules cluster M-13: calibrated and uncalibrated image . . . . . . 29

$4 \quad$ Camera head . . . . . . . . . . . . . . 34

5 Comparison of theoretical and experimental noise of master bias frame 38

6 Probability distribution of pixel values in a bias structure frame . . . 42

$7 \quad$ Temperature dependence of the bias offset $\ldots \ldots \ldots 44$

8 Typical bias frames for different temperatures . . . . . . . . 45

9 Temperature dependence of the bias noise $\ldots \ldots \ldots 46$

10 Temperature dependence of the stability and reproducibility of the bias offset $\ldots \ldots \ldots \ldots \ldots \ldots \ldots \ldots$

11 Typical dark frame .................. 49

12 Exposure time dependence of the dark count .......... 51 
13 Dark count populations . . . . . . . . . . . . . 52

14 Temperature dependence and doubling temperature of the dark count 54

15 Temperature dependence of the dark noise . . . . . . . . . 56

16 Exposure time dependence of the dark noise . . . . . . . . 58

17 Doubling temperature of the first hot pixel population . . . . . 61

18 Increased dark count caused by past light image . . . . . . . . 63

19 Decay of the dark offset after partially illuminating the chip . . . . 64

20 Image of a laser spot on a video screen with the corresponding dark

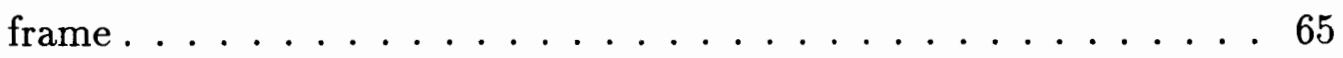

21 Decay of the dark offset past flat-field frames . . . . . . . . . . 67

22 Typical flat-field frame . . . . . . . . . . . . 73

23 Noise level in master bias frames . . . . . . . . . . . 76

24 Comparison of the two methods for master dark frame calibration . . 80

25 Difference of the squared noise of the two master flat-field frame calibration methods .................. 84

26 Comparison of the noise in a median or mean combined master bias

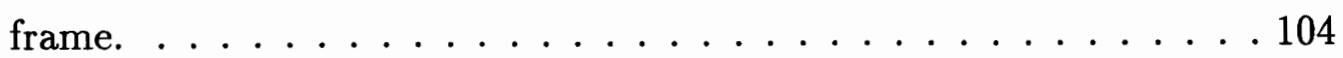




\section{Introduction}

\subsection{History of Light Detectors in Astronomy}

Astronomy, one of the oldest of sciences concerned with nature on the largest scale, is being aided by the newest developments in microelectronics, the most advanced technology of the very small. The advancement of knowledge in astronomical research was always constrained by the kinds of detectors available, therefore the history of astronomy is in large part the story of a continual search for more efficient and more accurate ways of measuring the meager light of stars[6].

In the 19th century, the eye was the only 'instrument' to gather the information given by the telescope. The eye is a very good light detector, perfectly tailored to its everyday uses, but it has its limitations for astronomy. Even though its efficiency is comparable to some modern light-detecting devices, it responds only to a limited range of colors. The eye can also discern subtle differences in light intensity but 
is a poor judge of absolute brightness. Its major disadvantage, however, is that it cannot store light for more than a few tenths of a second[6].

A big step forward was the invention of photography at the end of the 19th century. This invention offered such marked advantages that it quickly became the main detection method for astronomy. In spite of being less sensitive than the eye, it has the great advantage of accumulating light for a long time. This made it possible to measure the brightness of fainter stars. Nonetheless, there is still a limiting faintness beyond which an object cannot be detected on a photograph; for long exposures, the ever present background light from the night sky eventually saturates the entire emulsion. However, a further advantage is the wider range of sensitivity: from the ultraviolet region to the near-infrared. Over the years, several developments such as photo-electric systems and image intensifiers improved the performance of photography.

More recently the technology of television and electronic image amplification have been adapted to astronomy, both with the aim of combining the accuracy and unlimited exposure time of the photomultiplier with the extended field of view of the photographic plate. Various devices of this type have been proposed and tested, but in the last years, Charge-Coupled-Device cameras are getting more and more important for astronomy[6]. 


\subsection{Principles of a CCD Camera}

Recording a pattern of light is rather like measuring the distribution of rainfall over a field by setting out an array of buckets before the rain and afterward moving the buckets on to conveyer belts to a metering station where the amount of water in each bucket is recorded. In a Charge-Coupled-Device (CCD) camera the 'buckets' are electron-collecting zones of low electric potential created below an array of electrodes formed on the surface of a thin wafer of semiconducting silicon. The zones, called potential wells, are moved about within the device to an output amplifier by changing the voltage on the electrodes in a systematic manner[6].

When a photon strikes the silicon, it is very likely to give rise to a paired entity consisting of a displaced electron and the hole created by the temporary absence of the electron from the regular crystalline structure of the silicon. When a photon creates an electron-hole pair, the electron is immediately collected in the nearest potential well, whereas the hole is forced away from the well and eventually escapes into the substrate[6].

The CCD chip is divided into channels that are separated from one another by narrow barriers. Each channel is in turn subdivided along its length into pixels by a series of parallel electrodes (gates) which run across the device at right angles to the channels. Each row of the pixels is controlled by one set of gates. A picture is 
read out of the device by a succession of shifts through the imaging section, with all rows simultaneously moving one space at a time through the body of the device[6].

At each shift the last row of pixels passes out of the imaging section through an isolating region called a transfer gate into an output shift register. Before the next row is transferred, the information is moved along the output shift register, again one pixel at a time, to an amplifier at the end, where the charge in each pixel is measured. This final step constitutes a measurement of the original light intensity registered in each pixel. The technique for moving the electric charge is called 'charge coupling', which is how devices operating on this principle got their name. The basic physics of the process is quite linear: doubling the number of photons at any pixel will result in doubling the number of repelled electrons, until the potential well corresponding to that pixel is finally saturated[6].

\subsection{Comparison of CCD's and Other Light De-}

\section{tectors}

How does the CCD camera compare to other light detectors in the major criteria such as quantum efficiency, noise level, dynamic range, color response, photometric accuracy and field of view? 
Quantum efficiency is a measure of the sensitivity of a detector. An ideal detector would have a quantum efficiency of $100 \%$, that is, it would generate a measurable response for every photon that struck it without introducing noise and it would be sensitive to the light of all colors. In the real world, an ideal quantum efficiency is not reachable. In contrast to the eye and the kinds of emulsion commonly used in astronomy, which have a quantum efficiency of a few percent, CCD detectors can reach a quantum efficiency as high as $90 \%$. Since a more efficient detector yields more data, an observation made with such a detector can be done in less time or with a smaller telescope. A comparison of the quantum efficiency of a CCD-device to other light detectors is shown in Figure 1[6][9].

Most detectors add noise to the gathered signal. The light-sensitive particles in a photographic emulsion are not distributed uniformly. This produces the effect known as graininess, which hides features that are small or have a low contrast. In electronic detectors, noise is generated as a result of the constant thermal agitation of their constituent atoms or molecules. This thermal noise can be reduced by cooling the detector. Additionally, the electronic components add noise. Since the thermal noise can be reduced by subtracting a 'dark frame', electronic detectors are superior to photographic detectors[6][9]. 


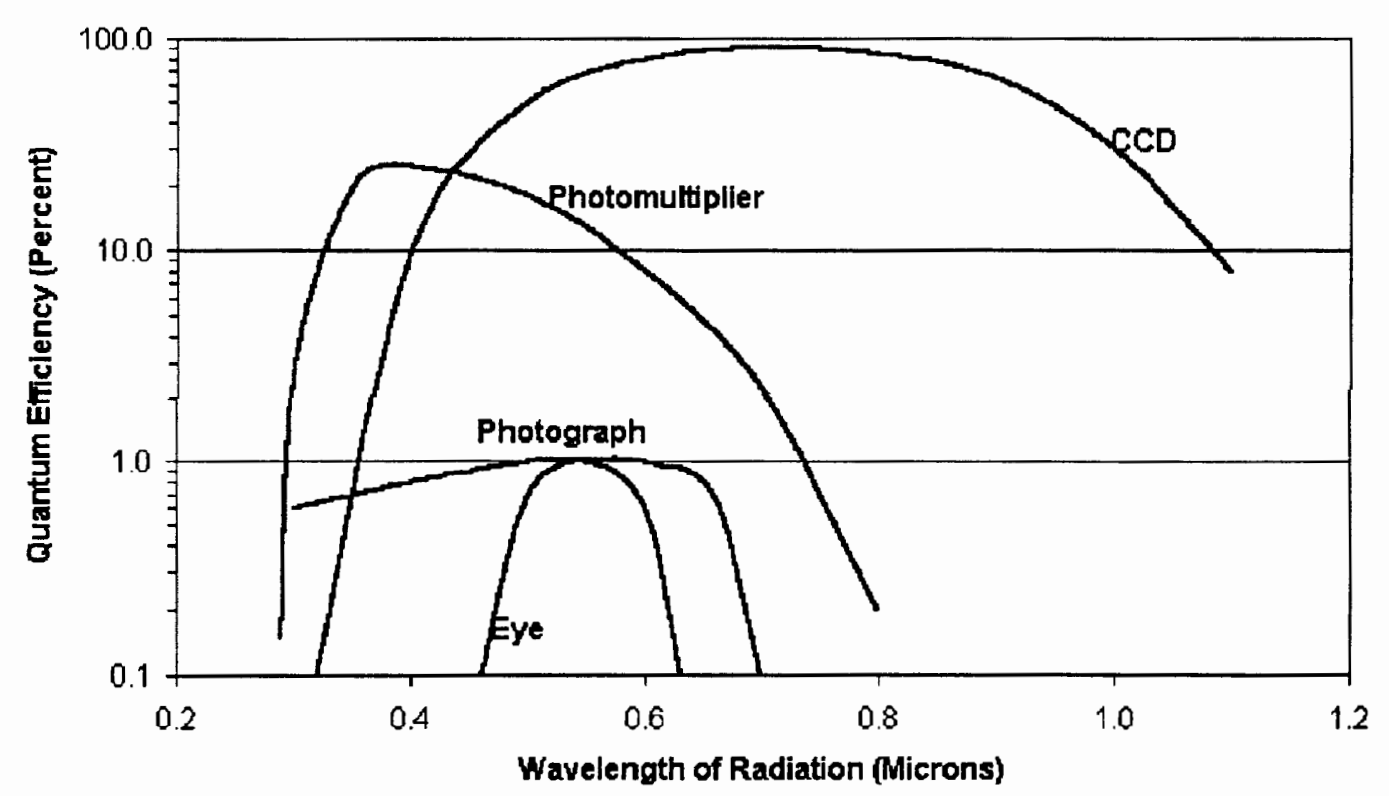

Figure 1: Comparison of the quantum efficiency of a CCD-device to other light detectors (see page 70 in [6]).

The dynamic range of a light detector is the ratio of the maximum detectable light intensity to the minimum detectable light intensity. The minimum level is usually determined by noise, the maximum by the fact that most detectors saturate in some way at high exposure times. By increasing the dynamic range of a detector more photons can be collected before saturation, the relative noise due to statistical photon processes can be reduced and fainter objects can be detected. Cooled CCD cameras have a very high dynamic range due to the fact that the limiting noise is very small[6][9].

An accurate measurement of brightness requires that the detector responds in a known and reproducible manner, so a given light input always yields the same 
output. CCD devices are highly linear devices. Photographs, on the other hand, are inherently nonlinear in several ways. Moreover, photographic plates can be used only once, and their characteristics are not reproducible, so that for the highest attainable photometric accuracy each plate must be individually calibrated; which is an inaccurate process[6][9].

The relatively small size of most CCD-chips is a disadvantage. It is not always possible to have the minimum of three reference stars needed for data reduction in the image. Compared to some photographic plates as big as several square meters, the CCD-chip looks small with its several square centimeters[9].

It is apparent that CCD cameras are a powerful addition to the astronomer's tools. In areas requiring high accuracy such as detecting faint stars, they are superior to other light detectors used in astronomy. The stability and linearity of CCD measurements provide a noticeable improvement in the general consistency of observations in comparison to photographic data. 


\section{Part I}

\section{Theoretical Background: Noise}

\section{and Repeatable Patterns in CCD}

\section{Images}




\section{Chapter 1}

\section{Repeatable Patterns}

The appearance of CCD cameras opened a door to the world of astronomy. Never before was it this easy and fast to obtain high quality images from stars and galaxies. But nevertheless, the uncertainty in signals makes life difficult for everyone who uses CCD cameras. A major goal is to keep this uncertainty small in order to obtain a signal that is as precise as possible. In most cases, the measured signal (raw signal) is different than the signal one wants to measure (real signal). The reason for this is, that the real signal is changed by counts added by charging the chip ('bias count') or the count accumulated due to thermal excitation ('dark count'). Fortunately, these effects are reasonable repeatable, so it is possible to remove them from the raw signal in order to obtain a cleaner signal. 
Several factors affect pixel values. Some factors will increase or decrease the measured intensity of two different pixels identically, whereas other factors affect pixels in different ways. Some factors depend on the exposure time, while others only add an offset. If the added count does not depend on the exposure time, we can subtract the added count and obtain a corrected image; if it depends on the exposure time, then the effect is multiplicative and we have to divide by a correction factor. If we want to calibrate the image without unnecessarily increasing the noise, we have to take all these effects into account[13].

Another important factor is the order in which the photons are affected as they travel from the source to becoming a digital image. Let us look at a light beam which is on its way through the telescope onto the camera until the image is read out of the detector[13].

- The light striking the telescope objective builds up a total signal $S_{\text {real }}$ proportional to the brightness of the light $R_{0}$ and the exposure time $t_{0}[13]$.

- The optical systems have varying degrees of vignetting, which reduce the intensity of the off-axis beam. Vignetting $(V)$ is a multiplicative effect[13].

- Dust on filters and optical windows reduce the intensity of a beam. These dust specks are imaged out of focus on the sensor and appear as shadows. With an unobstructed refractor the shadow looks like a disk, whereas a telescope 
with a central obstruction produces a doughnut. Dust shadowing $(s)$ is a multiplicative effect, as well (see Figure 22)[13].

- The shutter in a CCD camera travels at a finite speed and can produce an uneven exposure. This can cause slightly different exposure times for different pixels. With short exposures, this difference can be a significant percentage of the total exposure[13].

- Different pixels have in general different sensitivities. The amount of a signal recorded by a pixel depends upon its quantum efficiency $q$. It is also, as vignetting and dust shadowing, a multiplicative factor[13].

- Even in the absence of light, the pixels of a CCD will accumulate a signal due to the inescapable and random motions of electrons within the chip. The dark count signal $(D)$ is dependent on the exposure time, but not necessarily proportional to it. The time $t_{D}$ the dark count accrues in the detector is slightly larger than the real exposure time. The dark count is an additive factor and its contribution can be removed by subtracting a correction count[13].

- A CCD has to be operated in a charged state in order to detect and collect light. Since a false signal $(B)$ is created by this bias, it has to be removed by subtracting a correction count from the raw signal[13]. 
Given the conditions above, the raw signal can be described with the following equation[13].

$$
\begin{aligned}
S_{\text {raw }} & =s \cdot q \cdot V \cdot S_{\text {real }}+D+B \\
F & =s \cdot q \cdot V
\end{aligned}
$$

where $S_{\text {raw }}$ is the measured signal. The question now is how can we extract the real signal, $S_{\text {real }}$, from the raw signal? The correct way to remove each effect is to work the equations backward, from right to left, undoing each effect in the reverse order in which it happened: First we have to subtract the bias count and the dark count, then we have to divide by a multiplicative factor [13]. In practice, we do this by subtracting a dark frame $(\hat{D})$ and a bias frame $(\hat{B})$ and dividing by a flat field frame $(\hat{F})$.

$$
S_{c a l}=\frac{S_{\text {measured }}-\hat{D}-\hat{B}}{\hat{F}}
$$

In the ideal case, $S_{\text {cal }}$ would be equal to $S_{\text {real }}$, and here is where the problem starts: There is noise in the signals, therefore $\hat{D}, \hat{B}$ and $\hat{F}$ are only estimates for $D, B$ and $F$, respectively. 


\section{Chapter 2}

\section{Noise in Images}

Uncertainty in measureinents can be divided into two groups: those of a systematic and those of a statistical nature. In this chapter we will explore the nature and sources of the statistical and random uncertainty, the noise, and especially, how noise impacts a CCD image.

\subsection{The Statistical Nature of Noise}

The general term 'noise' refers to any process that contributes to errors of measurements or distortions of information. In astronomy, there is an ultimate source of noise that cannot be overcome: Light is quantized in the form of photons whose arrival at any point in time and space is represented statistically by a characteristic distribution function; hence, the number of photons that strike even a uniformly 
illuminated detector differs from area to area in a given time interval. This means that, in general equal signals differ slightly even if they are detected under identical conditions. Large deviations occur less often than small ones, and very, very large deviations almost never occur. The measured signals are scattered around an average value (mean). In general, we can characterize this distribution with the Gaussian probability distribution $(F)$, named for the German mathematician/astronomer, Carl Friedrich Gauss (1777-1855), who first derived it mathematically[10][6].

$$
F(x)=e^{\frac{(x-m)^{2}}{2 \sigma^{2}}}
$$

In this equation, $F(x)$ is the probability that the measured quantity will have the value $x, m$ is the average of the expected values (mean), and $\sigma$ is the standard deviation. Mathematically, $68 \%$ of the area enclosed by the curve is within one standard deviation of the mean and $90 \%$ within two standard deviations. This means that for a measured signal, which can be described by the Gaussian curve, there is a 68 percentage probability that the measured value is within one standard deviation from the true mean. The values corresponding to this 68 percent confidence level are usually those being quoted when a measurement is stated as being some value \pm uncertainty[10].

It is important to distinguish between noise and a repeatable pattern added to or multiplied by a signal, e.g., if the same count $c$ is always added to the real signal, 
it can be removed from a measured signal by subtracting it, but if the signal has the noise $c$, the noise cannot be removed since the specific error in one measured signal is unknown.

\subsection{The Sources of Noise}

Some of the noise that arises in a CCD image is from the fundamental properties of light itself. Due to the quantum property of light, the photons arrive at a detector in sporadic bursts, even from a perfectly constant source. This noise is equal to the square root of the signal and is named Poisson noise after the French mathematician Simeon Poisson (1781-1840). Therefore, signals detected under the same conditions have noise which is equal to the square root of the number of counted photons. The Poisson distribution looks much like the Gaussian distribution, but its width is determined by the square root of the total number of counts[10].

There are additional sources of noise in a CCD image that have to be taken into account:

- Readout noise: When the signal generated by light falling on a CCD is collected, amplified and converted to a digital value, noise is added at each step of the process[10]. 
- Background noise: The sky background is another source of noise in astronomical images. This can be caused by the natural skyglow, moonlight, or light pollution in urban areas. The background noise plays a minor role for bright stars, but for faint stars it is not negligible and it is essential that it be removed[10].

- Processing noise: Whenever an image is processed (e.g. when the dark count or the bias count is subtracted), the noise will be increased, no matter how good the calibration images are[10]. It is possible to minimize this added noise with a few strategies which will be mentioned in part II of this thesis.

In general, independent noises such as those mentioned above add quadratically. This means that the square of the total noise is equal to the sum of the squares of the individual noises.

$$
N^{2}=N_{1}^{2}+N_{2}^{2}+N_{3}^{2}+\cdots
$$

Even though the signal might decrease through processing, e.g., by subtracting a count, the noise will always add quadratically.

\subsection{Signal-to-Noise Ratio $\frac{S}{N}$}

The signal-to-noise ratio, $\frac{S}{N}$, is a very good measure to evaluate the accuracy of the signal. The higher the $\frac{S}{N}$ is, the easier is it to distinguish between the brightness 
of signals measured from two pixels next to each other, e.g., in order to get useful results in the detection of faint stars it is essential to increase the $\frac{S}{N}$ as much as possible.

As mentioned before, the light count can be characterized by a Poisson distribution. Since the standard deviation of a Poisson distribution is equal to the square root of the mean, the number of photons recorded in a given amount of time is uncertain by at least the square root of the number that has been collected. The $\frac{S}{N}$ is at most equal to $\frac{S}{\sqrt{S}}$, or $\sqrt{S}$. It can never surpass nature's maximum value of $\sqrt{S}$, which happens in the case of pure Poisson noise[10].

\subsection{Image Arithmetic}

Before CCD images are ready for display and measurement, we usually apply a series of standard image-processing procedures to remove bias count and dark count, and also to take variations in pixel-to-pixel sensitivity into account. Because these procedures modify the signal and the noise of the original image, it is important to know how each step affects the final results. But it is important to note that it does not matter which procedure we use, the noise never decreases. It always increases or at best, a very rare case, it remains unchanged. If noise comes from several independent sources, then it adds quadratically (see Equation (5)). We have to take this into account whenever we do image processing. Each procedure will be 
described in the following section. Table 1 gives an overview of the signal and noise levels in image arithmetic[11].

\subsubsection{Addition}

Adding multiple exposures is a common procedure in astronomy. The signals are simply added, but how does the signal-to-noise ratio change? If we use Equation (5) we can determine the $\frac{S}{N}$ :

$$
\begin{aligned}
\frac{S}{N}= & \frac{S_{1}+S_{2}+\ldots}{\sqrt{N_{1}^{2}+N_{2}^{2}+\ldots}} \\
= & \frac{S_{1}}{\sqrt{N_{1}^{2}+N_{2}^{2}+\ldots}}+\frac{S_{2}}{\sqrt{N_{1}^{2}+N_{2}^{2}+\ldots}}+\ldots \\
= & \frac{\frac{S_{1}}{N_{1}}}{\sqrt{1+\left(\frac{N_{2}}{N_{1}}\right)^{2}+\left(\frac{N_{3}}{N_{1}}\right)^{2}+\ldots}}+ \\
& \frac{\frac{S_{2}}{N_{2}}}{\sqrt{1+\left(\frac{N_{1}}{N_{2}}\right)^{2}+\left(\frac{N_{3}}{N_{2}}\right)^{2}+\ldots}}+\ldots
\end{aligned}
$$

We have two interesting cases that can be considered at this point. The first is, what happens when we add similar images? If the images have the same exposure time and are taken under similar conditions, they will have similar signal and noise levels. Mathematically speaking, we get:

$$
S_{1}=S_{2}=S_{3}=\ldots \text { and }
$$




$$
N_{1}=N_{2}=N_{3}=\ldots
$$

and we can plug that in Equation (6) and get

$$
\frac{S}{N}=\sqrt{n} \frac{S_{1}}{N_{1}}
$$

This shows that the signal-to-noise ratio of the sum of the images increases proportional to the square root of the number of images added[11].

The second interesting case is whether it is an advantage to add an image with a poor $\frac{S}{N}$ to an image with a good $\frac{S}{N}$ ? Let's consider an example: two images with the same signal but one has twice as much noise as the second, or:

$$
\begin{aligned}
& S_{1}=S_{2} \\
& 2 \cdot N_{1}=N_{2}
\end{aligned}
$$

Using Equation (6) we can estimate the $\frac{S}{N}$ of the resulting image.

$$
\begin{aligned}
\frac{S}{N} & =\frac{\frac{S_{1}}{N_{1}}}{\sqrt{1+\left(\frac{N_{2}}{N_{1}}\right)^{2}}}+\frac{\frac{S_{2}}{N_{2}}}{\sqrt{1+\left(\frac{N_{1}}{N_{2}}\right)^{2}}} \\
& =\frac{\frac{S_{1}}{N_{1}}}{\sqrt{1+2^{2}}}+\frac{\frac{S_{1}}{2 N_{1}}}{\sqrt{1+\left(\frac{1}{2}\right)^{2}}} \\
& <\frac{1}{2} \frac{S_{1}}{N_{1}}+\frac{1}{2} \frac{S_{1}}{N_{1}} \\
& <\frac{S_{1}}{N_{1}}
\end{aligned}
$$


Note that the resulting image has a signal-to-noise ratio smaller than the original image with the larger signal-to-noise ratio. It can be said as a rule of thumb, that it is not advantageous to add images with different noise levels[11].

\subsubsection{Subtraction}

When we process images we subtract bias and dark frames, and we sometimes subtract background levels for the sky. What happens when one image is subtracted from another?

$$
\frac{S}{N}=\frac{S_{1}-S_{2}-\ldots}{\sqrt{N_{1}^{2}+N_{2}^{2}+\ldots}}
$$

Although the signals are subtracted, the noise still adds quadratically. If the noise in the subtracted image is small, the degradation of the image is minor. This does imply, however, that it is advantageous to get calibration frames with very large signal-to-noise ratios[11].

\subsubsection{Multiplication}

Multiplication of two signals leads to the following signal levels and noise levels:

$$
S \quad=S_{1} * S_{2}
$$




$$
N^{2} \quad=S_{1}^{2} \cdot N_{2}^{2}+S_{2}^{2} \cdot N_{1}^{2}
$$

The signal-to-noise ratio can be determined

$$
\frac{S}{N}=\frac{S_{1} \cdot S_{2}}{\sqrt{S_{1}^{2} \cdot N_{2}^{2}+S_{2}^{2} \cdot N_{1}^{2}}}
$$

For equal signal levels and noise levels we can simplify Equation (12) to

$$
\begin{aligned}
\frac{S}{N} & \approx \frac{S_{1} \cdot S_{1}}{\sqrt{S_{1}^{2} \cdot N_{1}^{2}+S_{1}^{2} \cdot N_{1}^{2}}} \\
& \approx \frac{1}{\sqrt{2}} \cdot \frac{S_{1}}{N_{1}}
\end{aligned}
$$

The signal-to-noise ratio decreases for this special case by the factor of $\sqrt{2}$.

\subsubsection{Division}

To compensate for the multiplicative spatial nonuniformity and to obtain the uniform response of an ideal detector on an ideal telescope, we divide the image by a flat-field frame. Once again, processing an image degrades the signal-to-noise ratio of the resulting image, but in this case, the algebra is more involved. How can we determine the total noise? The squared total noise is again the sum of the squared individual noises, but this time the terms have coefficients. The noise from the flatfield $\left(N_{F}\right)$ is weighted with the ratio of the uncalibrated, raw signal $\left(S_{\text {raw }}\right)$ and the flat-field signal $(F)$. This means that if the flat-field signal is much larger than the 
raw signal, $N_{F}$ contributes very little to the total noise. Additionally, the sum of the squared raw signal noise $\left(N_{S, \text { raw }}\right)$ and the weighted $N_{F}$ has to be divided through the flat-field signal. This yields the following equation for the noise $N_{S, \text { final }}$ of the final signal $\left(S_{\text {final }}\right)$ :

$$
N_{S, f \text { inal }}^{2}=\frac{N_{S, \text { raw }}^{2}+\frac{S_{\text {anw }}^{2}}{F^{2}} \cdot N_{F}^{2}}{F^{2}}
$$

The total signal is just the ratio of the two signals.

$$
S_{\text {final }}=\frac{S_{\text {raw }}}{F}
$$

We can now use (14) and (15) to determine the $\frac{S}{N}$

$$
\begin{aligned}
\frac{S_{\text {final }}^{2}}{N_{S, f \text { inal }}^{2}} & =\frac{S_{\text {raw }}^{2}}{F^{2}} \cdot\left(\frac{N_{S, \text { raw }}^{2}+\frac{S_{\text {raw }}^{2}}{F^{2}} N_{F}^{2}}{F^{2}}\right)^{-1} \\
& =\frac{1}{\frac{1}{\left(\frac{S_{\text {raw }}}{N_{S, \text { raw }}}\right)^{2}}+\frac{1}{\left(\frac{F}{N_{F}}\right)^{2}}}
\end{aligned}
$$

The reduced form in (16) shows, that it is not only very important how large the signal-to-noise ratio of the flat-field image is, but it is even more important how good it is compared to the signal-to-noise ratio of the original image. As a rule of thumb, we can say the signal-to-noise ratio of the processed image is slightly smaller than the poorest signal-to-noise ratio of the processing images, if they have different signal-to-noise ratio's. 
In general, dividing through an image works best when the calibration frame has a signal-to-noise ratio larger than that of any pixel of interest in the image that is processed. The best way to reach a large signal-to-noise ratio for the calibration frame is to combine several images into a master frame[11].

\subsubsection{Multiplication with a Constant}

Sometimes it is necessary to normalize images. In this case we multiply the image with a constant. The result works out nicely:

$$
\frac{S}{N}=\frac{k S_{1}}{k N_{1}}=\frac{S_{1}}{N_{1}}
$$

Because the constant affects the signal and the noise in exactly the same manner, the constant terms cancel and there is no net effect on the result.

\subsubsection{Combining Images}

Image processing, like calibration, always adds noise. To minimize the degradation by calibration, we should use a calibration image that has a high signal-to-noise ratio. This is best accomplished by taking many frames and combining them into a master. But why should we not take a calibration image with a longer exposure time? The advantage of multiple exposures is that a CCD's response is less apt to 


\begin{tabular}{|l|c|c|}
\hline Arithmetic & Signal level & Noise level \\
\hline Addition & $S=S_{1}+S_{2}$ & $N^{2}=N_{1}^{2}+N_{2}^{2}$ \\
\hline Subtraction & $S=S_{1}-S_{2}$ & $N^{2}=N_{1}^{2}+N_{2}^{2}$ \\
\hline Multiplication & $S=S_{1} \cdot S_{2}$ & $N^{2}=S_{2}^{2} \cdot N_{1}^{2}+S_{1}^{2} \cdot N_{2}^{2}$ \\
\hline Division & $S=\frac{S_{1}}{S_{2}}$ & $N^{2}=\frac{N_{1}^{2}+\frac{S_{1}^{2}}{S_{2}^{2}} N_{2}^{2}}{S_{2}^{2}}$ \\
\hline Multiplication with a constant c & $S=c \cdot S_{1}$ & $N^{2}=c^{2} \cdot N_{1}^{2}$ \\
\hline Combining & $S=\frac{1}{n} \sum_{i=1}^{n} S_{i}$ & $N^{2}=\frac{1}{n^{2}} \sum_{i=1}^{n} N_{i}^{2}$ \\
\hline
\end{tabular}

Table 1: Overview of signal and noise levels in image arithmetic become nonlinear, which can happen if the pixels approach saturation. Furthermore, multiple frames aid in removing such random features as cosmic-ray detections.

In most cases we combine images with similar signal and noise levels. If this is the case, then the combining images can be split into two steps:

- Adding $n$ frames:

$$
\begin{aligned}
S^{\prime} & =\sum_{i=1}^{n} S_{i} \\
N^{\prime 2} & =\sum_{i=1}^{n} N_{i}^{2}
\end{aligned}
$$

- And normalizing $S^{\prime}$ to $\bar{S}$ by dividing by $n$ :

$$
\begin{aligned}
\bar{S} & =\frac{S^{\prime}}{n} \\
\bar{N} & =\frac{1}{n} \sqrt{\sum_{i=1}^{n} N_{i}^{2}}
\end{aligned}
$$


In most cases we combine images with similar signal and noise levels. If this is the case, then the effect these two steps have on the $\frac{\bar{S}}{N}$ based on the $\frac{S_{i}}{N_{i}}$ of the original pictures is as follows:

$$
\begin{aligned}
\frac{S^{\prime}}{N^{\prime}} & \approx \sqrt{n} \frac{S_{i}}{N_{i}} \\
\text { and } \frac{S^{\prime}}{N^{\prime}} & =\frac{\bar{S}}{\bar{N}}
\end{aligned}
$$

where $i \epsilon\{1 . . n\}$. If we combine (22) and (23), we get

$$
\frac{\bar{S}}{\bar{N}} \approx \sqrt{n} \frac{S_{i}}{N_{i}}
$$

and because $\bar{S} \approx S_{i}$ we can write

$$
\begin{aligned}
\frac{1}{\bar{N}} & \approx \sqrt{n} \frac{1}{N_{i}} \text { or } \\
\bar{N} & \approx \frac{N_{i}}{\sqrt{n}}
\end{aligned}
$$

So, if we combine $n$ frames, we get a frame with the same signal, but with noise decreased by the factor $\sqrt{n}$. 


\subsection{Noise in the Calibrated Signal}

Armed with this knowledge, we can estimate the noise in a raw signal and in a calibrated signal. We can literally describe the raw signal $S_{\text {raw }}$ (see Equation (1)) by

$$
S_{\text {raw }}=F \cdot S_{\text {real }}+D+B
$$

Let us consider the noise in this raw signal. The problem is, that there is not only noise in the real signal, but there is also noise in the dark count and the bias count. Since these noises are all caused by independent sources, we can determine the noise by

$$
N_{S, \text { raw }}^{2}=F^{2} \cdot N_{S, \text { real }}^{2}+N_{F}^{2} \cdot S_{\text {real }}^{2}+N_{D}^{2}+N_{B}^{2}
$$

The first calibration steps are to subtract the bias count and the dark count. The corresponding signal and noise levels are

$$
\begin{aligned}
S_{c a l 1} & =S_{\text {raw }}-\hat{D}-\hat{B} \\
& =\left(F \cdot S_{\text {real }}+D+B\right)_{\text {raw }}-\hat{D}-\hat{B} \\
N_{c a l 1}^{2} \quad & N_{S, \text { raw }}^{2}+N_{\hat{D}}^{2}+N_{\hat{B}}^{2} \\
& =\left(F^{2} \cdot N_{S, \text { real }}^{2}+N_{F}^{2} \cdot S_{\text {real }}^{2}+N_{D}^{2}+N_{B}^{2}\right)_{r a w}+N_{\hat{D}}^{2}+N_{\hat{B}}^{2}
\end{aligned}
$$


where $S_{c a l 1}$ and $N_{\text {cal1 }}$ represent the signal and noise level after the first calibration steps are done. Similarily, flat fielding (dividing by $\hat{F}$ ) will decrease the signal-tonoise ratio since there is noise as well in the flat-field frame. The total noise can then be determined:

$$
\begin{aligned}
S_{c a l 2} & =\frac{S_{c a l 1}}{\hat{F}} \\
N_{c a l 2} & =\frac{N_{c a l 1}^{2}+\frac{S_{c a l 1}^{2}}{\hat{F}^{2}} N_{\hat{F}}^{2}}{\hat{F}^{2}}
\end{aligned}
$$

where $S_{c a l 2}$ and $N_{c a l 2}$ is the final signal and its noise level. In Figure 2 and Figure 3 examples are shown which demonstrate the differences of an uncalibrated and a calibrated image.

Functionally, it is possible to split the total noise in a calibrated image into two parts. On the one hand, there is the part corresponding to the raw signal, $N_{S, \text { raw }}$. Exposure time, temperature, equipment and other factors have influence on this part of the total noise. But this noise is fixed as soon as the image is taken. On the other hand all the calibration steps add noise. It is possible to minimize the altering of the noise caused by the calibration steps by carefully choosing and generating calibration frames with high signal-to-noise ratio after the images are taken. In part II, we will examine in detail how to apply the calibration frames considering trade-offs in imaging time, memory requirements and noise level. 

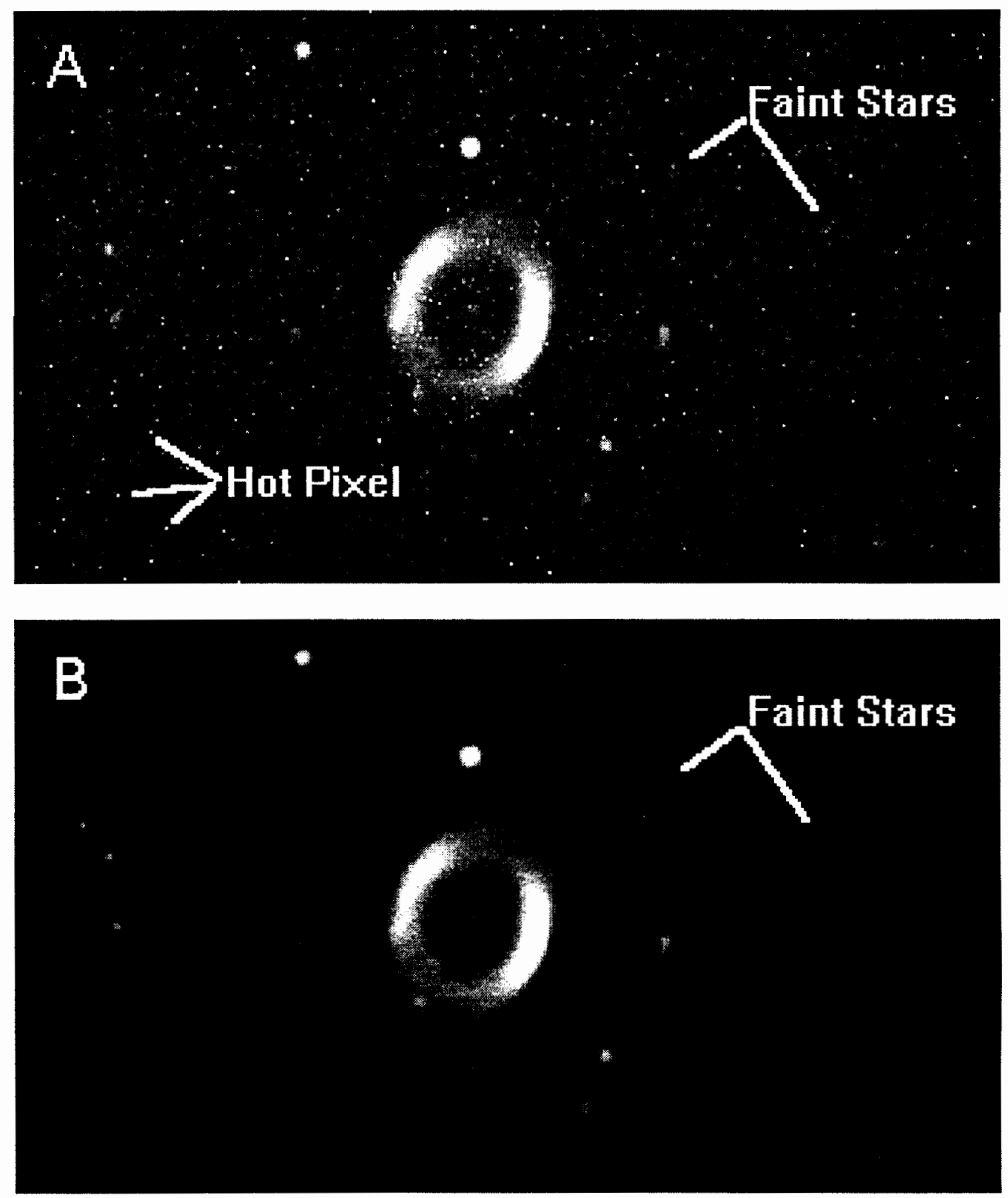

Figure 2: Image A shows an uncalibrated image of Ring Nebula M-57. In Image B the calibrated image, i.e., the dark frame and the bias frame are subtracted and then the image is divided by a flat field frame, is displayed. The image was taken at $-5^{\circ} \mathrm{C}$ and with a 240 second exposure time. The Ring Nebula M-57 is a planetary nebulae 4000 light-years away from our solar system. Its central star has a magnitude of 15 . 

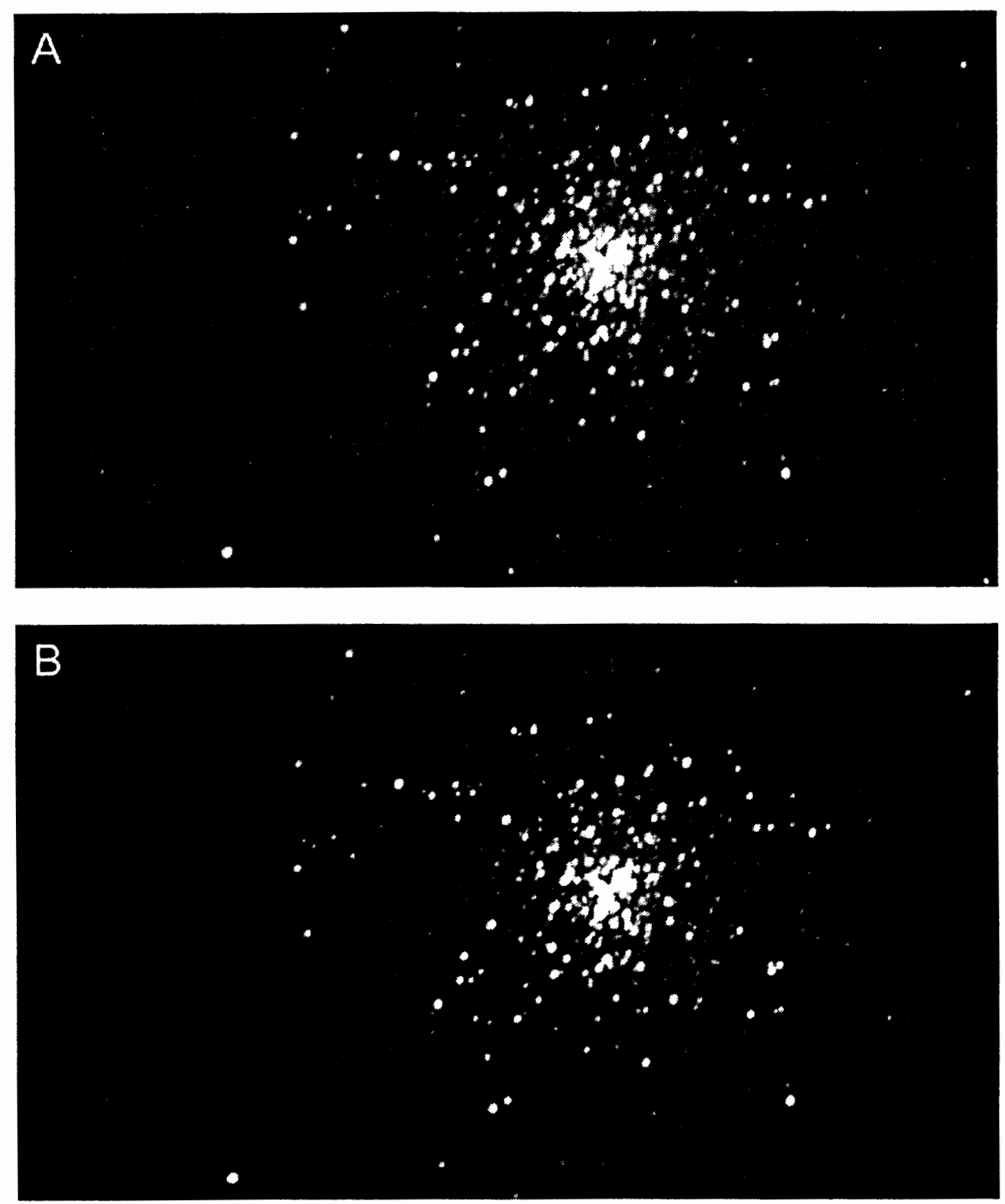

Figure 3: Image A shows an uncalibrated image of the Hercules Cluster M-13. In Image $\mathrm{B}$ the calibrated image is displayed. The image was taken at $-5^{\circ} \mathrm{C}$ and with a 120 second exposure time. M-13 is an irregular cluster which is about 500 million light-years from Earth. 


\section{Part II}

\section{Calibration of CCD Images}


Before we can use a CCD image it has to be calibrated in order to remove the dark and bias counts and to correct for the irregularities across the telescope's field of view as well as for the variations in the sensitivity of the CCD itself. Clearly, the calibration method has a significant impact on the quality of the final image and different calibration methods yield different results on the final image.

First of all, we should consider the order in which we correct the raw image with the calibration frames. The correct way is to remove each effect with the 'Last in - First out' method, this means that we undo each effect in the reverse order in which it happened. Correction for bias should be first, dark count second, and division by the flat-field frame for the multiplicative terms should be last.

It is essential to know more about the calibration images to develop a method of calibration that obtains a high quality image with the lowest possible noise. In the experiments done, the characteristics and properties of the calibration frames are examined. Furthermore, different methods for calibrating master frames were performed. This was done for both master dark frames and master flat-field frames. Using the method of master frame calibration, different possible night sessions are discussed for the trade-offs in imaging time, memory requirements, calibration time and noise level. A way to estimate the noise level in a calibrated image is described. 


\section{Chapter 3}

\section{Instruments and Methods}

\subsection{Instruments}

\subsubsection{CCD-Device}

The CCD-Camera used was manufactured by Axiom Research, Inc., Tucson, Arizona. It is an AX-2 model with a Kodak KAF 1600-2 Sensor.

\section{Sensor}

The built-in sensor is a Kodak KAF 1600-2 Sensor with a pixel area of 14.0 × $9.3 \mathrm{~mm}$ and a pixel size of $9 \mu \mathrm{m}$. It is divided into 1536 imaging columns and 1024 imaging 
rows. According to the specifications the sensor has a well depth ${ }^{1}$ of 85,000 electrons, a dynamic range ${ }^{2}>76 \mathrm{db}$, a read noise $\mathrm{e}^{3}$ of $13-20$ electrons, a dark count at $25^{\circ} \mathrm{C}$ of $50 \mathrm{e}-/ \mathrm{sec} /$ pixel and a doubling temperature of $5-6^{\circ} \mathrm{C}$. A 16 bit A/D converter with correlated digital doubling sampling is used. The sampling rate is 50 kilopixel/second. The exposure time can be varied between 0.02 and 10400 seconds. A 16 bit, 3/4 length ISA bus card is used as the computer interface. The power is supplied by the computer power supply.

\section{Camera head}

The camera head used is an S-type model. It is made out of 6061-T6 aluminium, hard anodized with stainless steel fasteners. It connects to lenses and other instruments through a standard T-thread lens mount on the front of the body. The camera is described in Figure 4.

\section{Thermal Control System}

The camera combines active and passive thermal control designs to achieve cooling capacity and temperature stability. The active part of the system uses a one-stage

\footnotetext{
${ }^{1}$ The value is the minimum number of electrons over which the CCD response has less than $1 \%$ nonlinearity.

${ }^{2}$ The value $20 \log S_{\max } / \mathrm{R}$, where $S_{\max }$ is the signal measured at the maximum 'linear' well depth, and $\mathrm{R}$ is the sensor's readout noise, both measured in electrons.

${ }^{3}$ The stated value is attributable to the sensor only and is not measured from images.
} 


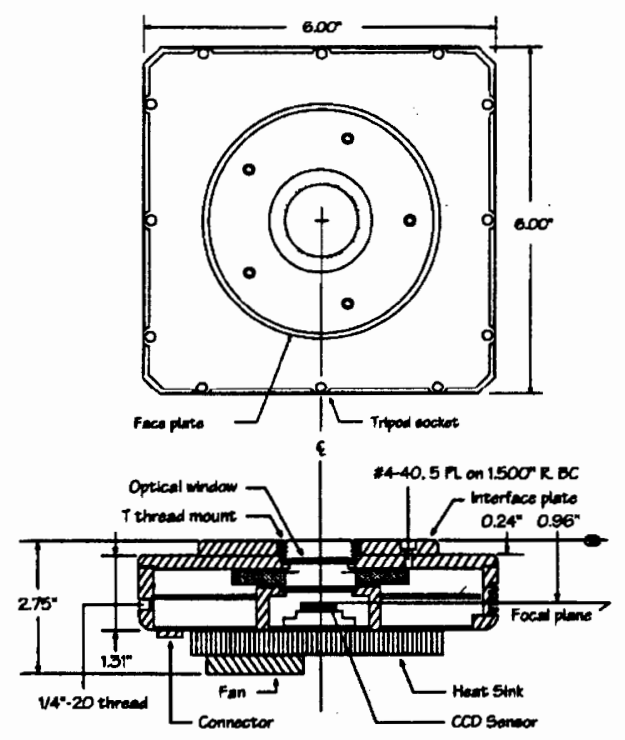

Figure 4: This figure shows a S-type camera head. On top, an outside front view is displayed. On the bottom, a cross sectional view is shown.

thermoelectric cooler with closed-loop stabilization through the Temperature Control Unit (TCU). When the cooler is turned on, the TCU continuously monitors the CCD sensor temperature and makes corrections to keep it stable within an uncertainty of approximately $\pm 0.1^{\circ} \mathrm{C}$. The software commands the TCU to ascend or descend. The maximum cooling capacity is $38^{\circ} \mathrm{C}$ below ambient temperature.

\subsubsection{Mira Software}

The software used was MIRA A/P (Microcomputer Image Reduction and Analysis software). MIRA comprises a family of powerful, full featured image processing software for astronomy and related areas of research. It is a 32-bit program that runs on Intel processors under the DOS operating systems. MIRA A/P provides a 
rich tool box of high and low level image processing functions which makes it easy to analyze any kind of images.

\subsubsection{Lab Setup}

The camera was connected to a computer with a Pentium processor (120 MHz), 16 MByte Memory and 1.6 GByte hard disk. This was sufficient to work fast and efficiently with the camera. A $28-200 \mathrm{~mm}$ Tokina SZ-X 282 lens was connected to the camera. For flat-fielding, a video screen and two lamps were used. Additionally, white paper was fixed in front of the lens in order to get a uniformly illuminated sensor. All data were collected with this setup except the star images, i.e., the images of the Ring Nebula M-57 and the Hercules Cluster M-13.

\subsubsection{Telescope Setup}

The CCD camera described in section 3.1.1 was connected to a Meade LX200 10 inch f/6.3 Schmidt-Cassegrain telescope and to the computer described in section 3.1.3. A Celestron C-5+ telescope was connected to a SBIG ST-7 CCD camera. The LX200 telescope was aligned to the Celestron, so that the Celestron/ST-7 setup could be used for the tracking. The tracking program is an integral part of the ST-7 operating system. The images of the Ring Nebula M-57 and the Hercules 
Cluster M-13 were taken with this setup which was located in Beaverton, a suburb in Portland, Oregon.

\subsection{Methods}

\subsubsection{Measuring the Noise of an Image}

Unfortunately, it is not possible to measure the noise of an image directly. So, how can we find out how much noise an image has? An easy and very accurate indirect way is to subtract two images taken under the same conditions (denoted as the 'subtraction method'). The two images, denoted as $I_{1}$ and $I_{2}$, should then have similar signal levels and noise levels. Therefore, the difference of the two images will have a mean of zero. Due to the fact that noise always adds quadratically, we can determine the noise $\left(N_{\text {diff }}\right)$ from the resulting image, denoted as difference frame Diff, with

$$
\begin{array}{ll}
\text { Diff } & =I_{1}-I_{2} \\
N_{d i f f}^{2} & =N_{I 1}^{2}+N_{I 2}^{2}
\end{array}
$$


and if the noise in the two images is similar, we can then determine the noise of the original images

$$
N_{I 1}^{2}=N_{I 2}^{2}=\frac{N_{d i f f}^{2}}{2}
$$

The following example shows the efficiency of this method. First, 30 bias frames are taken in a row. Experimentally, the noise $\left(N_{B, \operatorname{master}(n)}\right)$ of the master bias frame $\left(B_{\text {master }(n)}\right)$ is measured with the subtraction method mentioned above (subtracting only master bias frames combining equal number of frames), where $n$ is denoted as the number of bias frames used for the generation of the master bias frame.

$$
\begin{aligned}
& \operatorname{Diff} f_{\text {master(n),exp }}=B 1_{\text {master(n) }}-B 2_{\text {master }(n)} \\
& N_{D i f f, \operatorname{master}(n), \exp }^{2}=N_{B 1, \operatorname{master}(n)}^{2}+N_{B 2, \operatorname{master}(n)}^{2} \\
& =2 \cdot N_{B, \operatorname{master}(n)}^{2} \\
& =2 \cdot \frac{N_{B}^{2}}{n} \\
& N_{B, \operatorname{master}(n), \exp }^{2}=\frac{N_{D i f f, \operatorname{master}(n), e x p}^{2}}{2}
\end{aligned}
$$

The experimental value for the noise $\left(N_{B, \text { exp }}\right)$ in a single bias frame is $5.63 \pm 0.01$ counts. Using this experimentally measured noise of a single bias frame, the noise 
of a master bias frame can then theoretically be evaluated with the number $n$ of bias frames used:

$$
N_{B, \operatorname{master}(n), \text { theo }}^{2}=\frac{N_{B, e x p}^{2}}{n}
$$

A comparison of the experimental noise $N_{B, \operatorname{master}(n), \exp }$ and the theoretical noise $N_{B, \text { master(n),theo }}$ of master bias frames is shown in Figure 5.

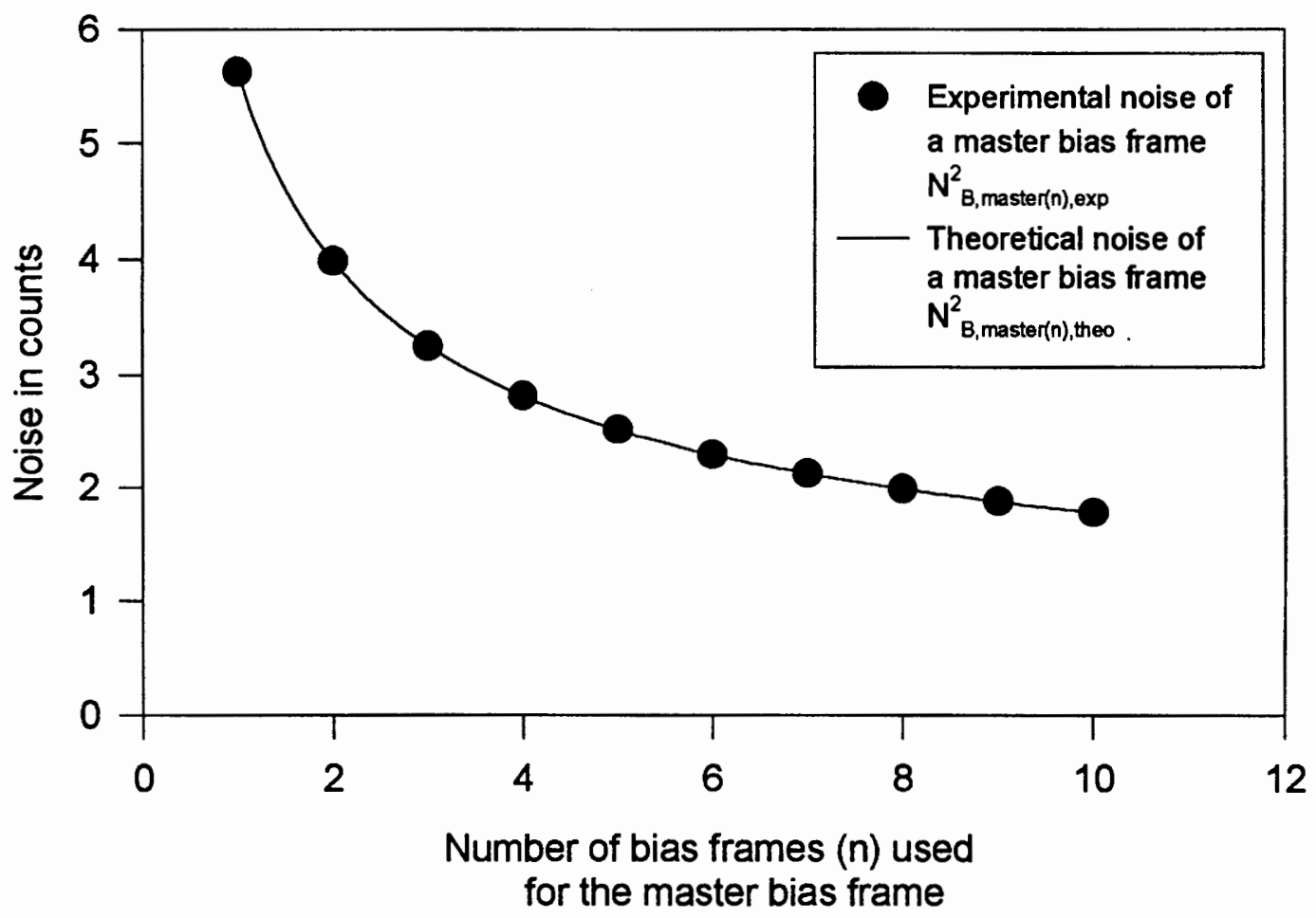

Figure 5: Comparison of theoretical and experimental noise of master bias frames. The experimental noise is measured with the subtraction method, the theoretical noise is calculated with Equation (38) using the measured noise of a single bias frame. The bias frames are taken at $-10^{\circ} \mathrm{C}$. 
With only the knowledge of the noise of single bias frames, it is possible to predict the noise of master bias frames very accurately. The nearly perfect match between experimental data gathered for this camera and theoretical values demonstrates the amazing accuracy with which it is possible to estimate the noise of the calibration frames with this method. This example shows two things:

- It is possible to indirectly measure the noise of an image very accurately.

- In image processing, the way the noise is processed is very predictable. As in the example above, the noise of an image combining similar images can be estimated using only the average noise of a single image.

\subsubsection{Experimental Procedures in the Lab}

All the data were collected by using the CCD-Camera described in section 3.1.1. In order to increase the processing speed and to save memory, a $392 \times 258$ pixel subframe instead of the full frame was used. The total number of 101136 pixels in this subframe was sufficiently large to do the statistical experiments. The master frames were processed by mean combining ${ }^{4}$ the single frames. The noise in the frames was determined by using the subtraction method described in section 3.2.1. Refer to figure caption for specific experimental set up.

\footnotetext{
${ }^{4}$ mean combining: averaging the pixel values of the same pixel over all frames.
} 


\section{Chapter 4}

\section{Bias Frames}

The bias count is caused by the charge applied to a CCD to activate its photoncollecting capacity, therefore it is present as a false signal in every image. Typically, the bias level drifts up and down with time as the camera's electronics change temperature. Thus, the measurements made immediately after an image is taken give the best estimate of the bias. In general, a bias frame contains a structure with the pixels in one part of the image containing a different average value than those from a different part. Therefore we can functionally split the raw bias count $\left(B_{\text {raw }}\right)$ into a noise-free bias offset $\left(B_{\text {offset }}\right)$ and a bias structure $\left(B_{\text {struc }}\right)$, that includes noise. For clarification in the notation: $B_{\text {raw }}$, the total bias count, varies from pixel 
to pixel, $B_{\text {offset }}$, the bias offset, is a constant that is the same for all pixels and $B_{s t r u c}$, the bias structure, again varies from pixel to pixel[13].

$$
B_{\text {raw }}=B_{\text {offset }}+B_{\text {struc }}
$$

It turns out the bias structure is very stable over a long period of time while the bias offset can drift. So, it is advantagous for some processing to separate $B_{\text {struc }}$ from $B_{o f f s e t}$ and correct them separately so as to achieve a better signal-to-noise ratio. It is easy to achieve the correct bias offset by calculating the mean over a sufficiently large area. It is critical to always use the same area for calculating the mean, otherwise any bias structure present will deteriorate the results. The bias structure can be obtained by subtracting the calculated bias offset from the raw bias frame. In Figure 6A the probability distribution of the bias structure count around the mean value of 0 is shown[13].

Since the bias count is not a Poisson count, the bias noise is not the square root of the bias count and we have to pay extra attention to the change of the noise for different bias counts. 

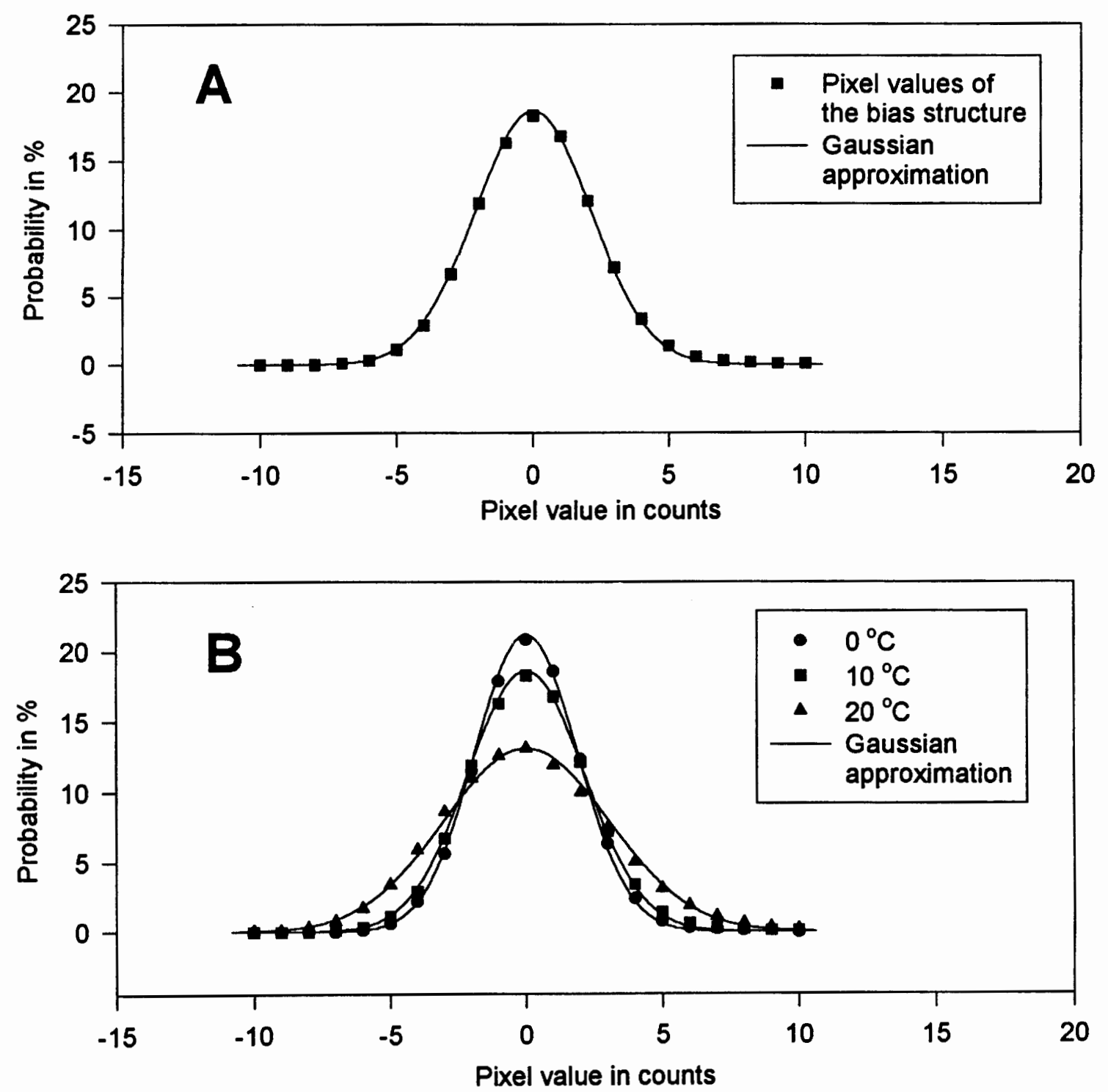

Figure 6: Graph A shows the probability that a pixel in a bias structure frame has a specific count. This bias structure frame combines 10 bias frames taken at $10^{\circ} \mathrm{C}$ for which the bias offset was removed. In Graph B, this probability distribution is shown for different temperatures. 


\subsection{Temperature}

The bias count of a CCD camera can drift up and down with time as the camera electronics change temperature. With the camera used, it was possible to cool the chip down to a maximal temperature difference of $38^{\circ} \mathrm{C}$.

The bias offset decreases as expected with decreasing temperature, but if the temperature decreases below $0^{\circ} \mathrm{C}$, the bias count increases again slightly. Overall, it can be said that the bias offset changes only slightly for temperatures below $10^{\circ} \mathrm{C}$ (see Figure 7).

Just as the bias offset is dependent upon temperature, the bias structure also exhibits a temperature dependence. This effect is apparent in Figure 6B, where the pixel value probability of the bias structure for different temperatures is shown. It can be recognized that the pixel values are uniformly distributed around the mean value of 0 with a Gaussian shape. Notice the importance in the trend as the plot narrows around the mean value with decreasing temperature.

The pixels with different values are not uniformly distributed across the frame, on the contrary, they form a structure. One common structure is attributable to the gradual buildup of the dark count during the read-out time. This structure can be recognized as an increase of the counts in the bias frames as we traverse from top to bottom (see Figure 8A). The effect is highly dependent on how large the dark count is (how warm the CCD Camera is and how much time the software needs to 
read-out the image). In Figure $8 \mathrm{~A}$ (CCD camera working at a temperature of $20^{\circ}$ C) this structure is obvious, whereas in Figure $8 \mathrm{~B}$ this effect does not occur in such a manner. In Figure $8 \mathrm{C}$, due to the low temperature $\left(-10^{\circ} \mathrm{C}\right)$, the dark count is very small (less than 1 count per second) and therefore its accumulation in the read-out process is negligible. The result is that the structure cannot be noticed any longer.

The vertical white pixel lines are another common structure. These white pixel lines are easily noticed in Figure $8 \mathrm{~A}$ and $8 \mathrm{~B}$, but they nearly disappear when the camera is cooled down. In Figure $8 \mathrm{C}$ very few tracks from the hot pixel lines can still be noticed.

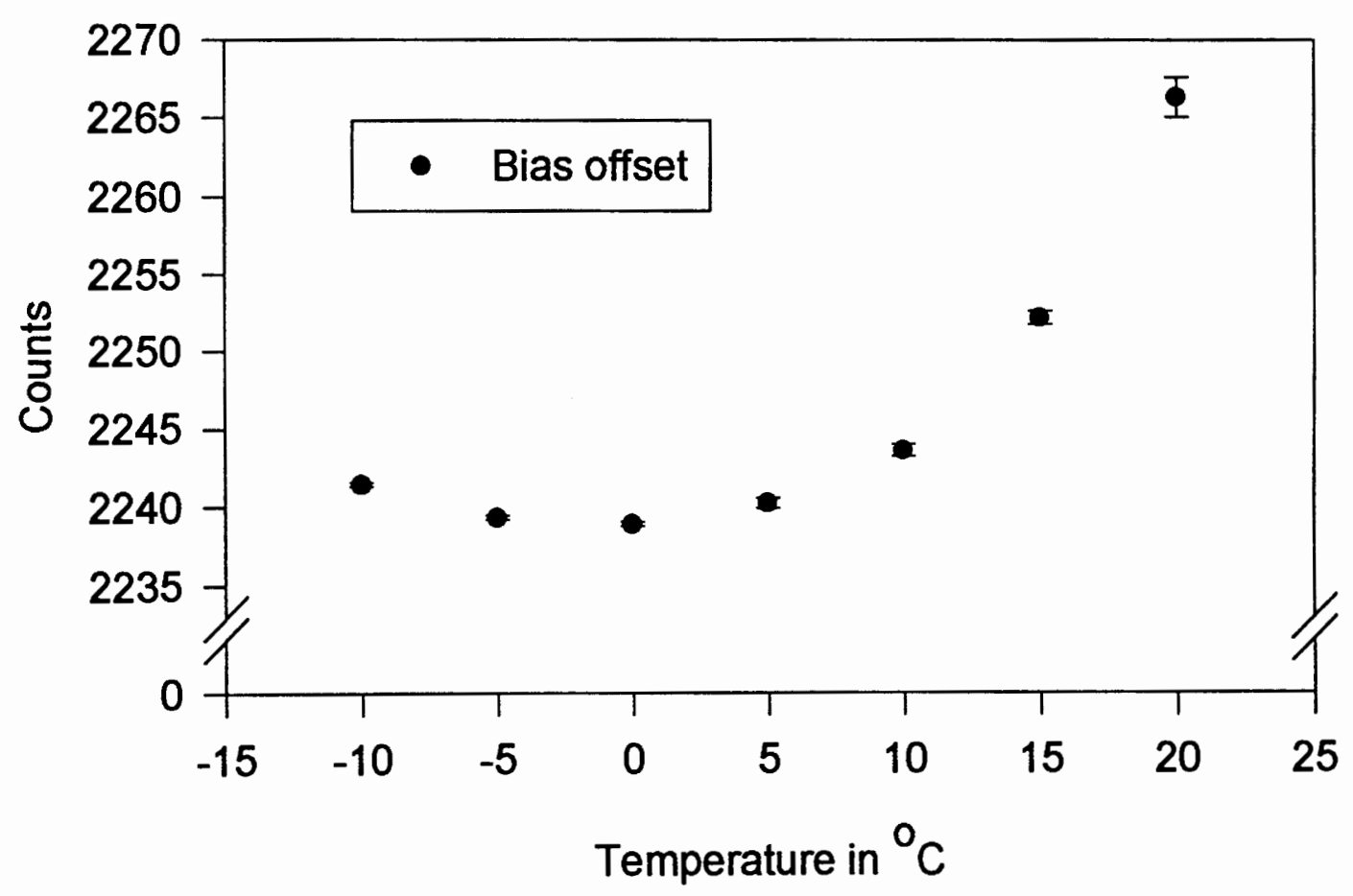

Figure 7: Temperature dependence of the bias offset. 

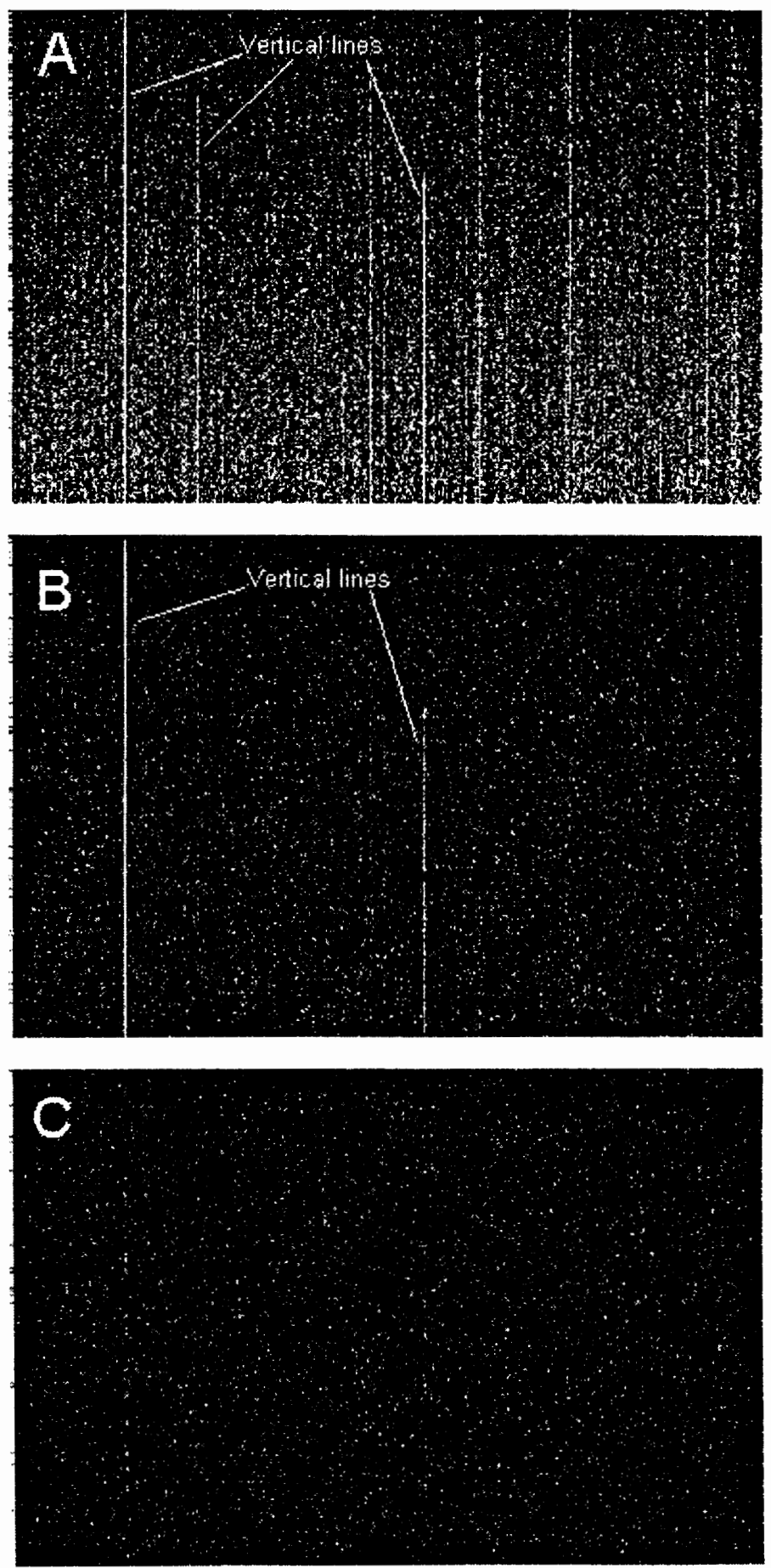

Figure 8: This Figure shows typical bias frames for different temperatures; $20^{\circ} \mathrm{C}, 5^{\circ} \mathrm{C}$ and $-10^{\circ} \mathrm{C}$ for the Graphs $\mathrm{A}, \mathrm{B}$ and $\mathrm{C}$, respectively. 


\subsubsection{Bias Noise}

The bias noise decreases with lower temperatures. One reason for this is that the gradual dark count build-up in the read-out process decreases as the temperature decreases, and therefore the corresponding noise decreases as well. Nevertheless, the effect is small (see Figure 9) and may be neglected.

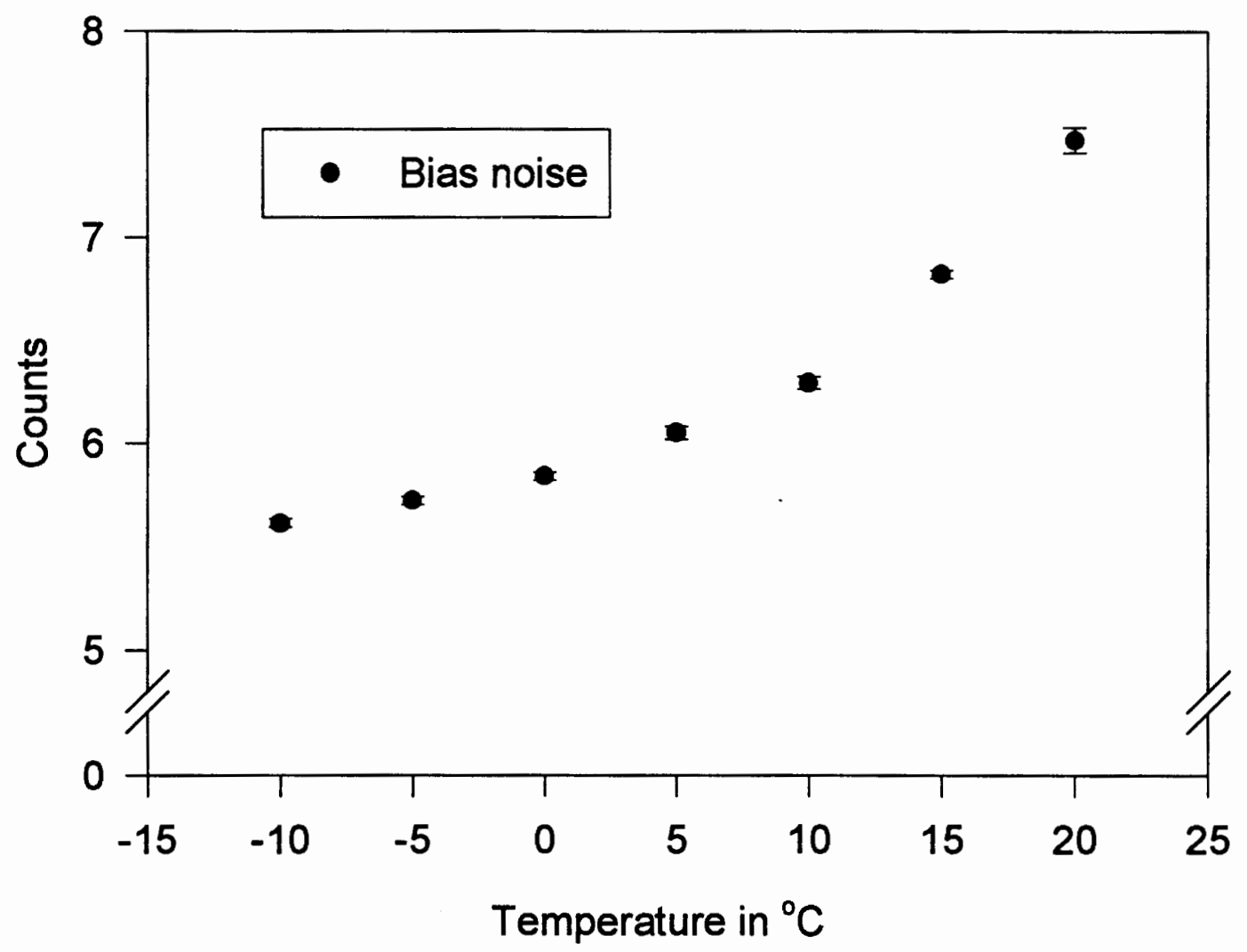

Figure 9: Temperature dependence of the bias noise. 


\subsection{Stability}

Another important question is how stable and reproducible is the bias count. The bias offset can drift with the temperature of the camera electronics. Figure 10 shows the bias offset of 20 bias frames for each temperature taken in a row. For low temperatures, the bias offset is nearly constant, whereas for high temperatures, the bias count drifts up and down around a mean value. It is necessary, and highly advantageous, to maintain a stable bias offset in order to accurately correct the bias count in image calibration.

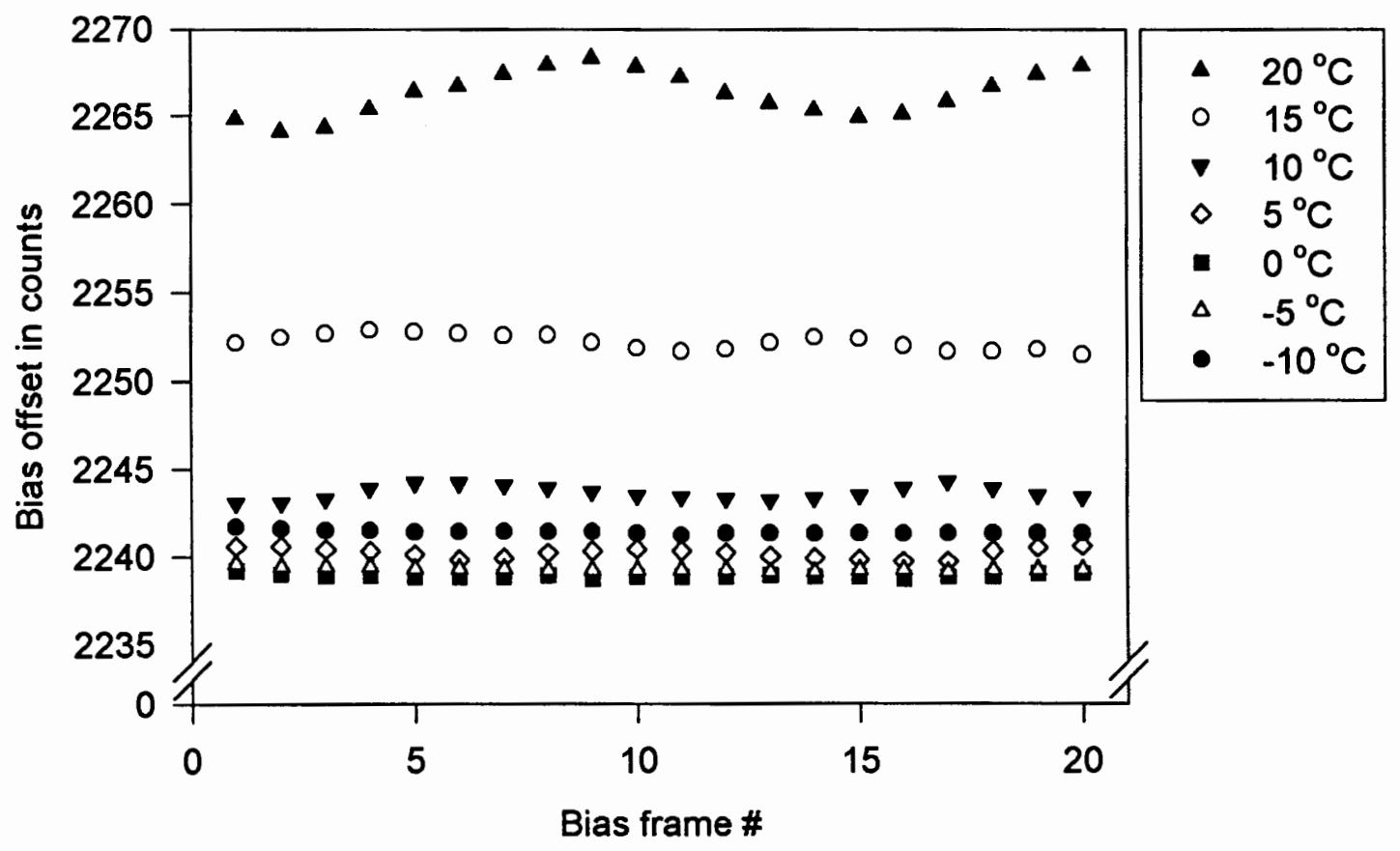

Figure 10: This Figure shows, how stable and reproducible the bias offset is over time. For each temperature, 20 bias frames were taken in a row. The mean of the bias count over the whole frame (bias offset) was calculated and plotted versus the bias frame number. 


\section{Chapter 5}

\section{Dark Frames}

Even in the absence of light, the pixels of a CCD will accumulate a signal proportional to the exposure duration. The random motions of electrons within the chip are the source of this dark or thermal count. It is essential to remove the dark count in order to process an accurately calibrated image.

The raw dark count in a randomly picked pixel can functionally be separated in a dark count $(D)$ and a bias count $(B)$.

$$
\begin{aligned}
D_{\text {raw }} & =(D+B)_{\text {raw }} \\
N_{D, \text { raw }}^{2} & =N_{D}^{2}+N_{B}^{2}
\end{aligned}
$$


We have to subtract a bias frame $(\hat{B})$ in order to obtain the real dark count. In other words, we have to 'calibrate' the calibration frame. The corresponding signal and noise levels can be determined:

$$
\begin{aligned}
D_{\text {cal }} & =(D+B)_{\text {raw }}-\hat{B} \\
N_{D, \text { raw }}^{2} & =N_{D}^{2}+N_{B}^{2}+N_{\hat{B}}^{2}
\end{aligned}
$$

A typical dark frame is shown in Figure 11.

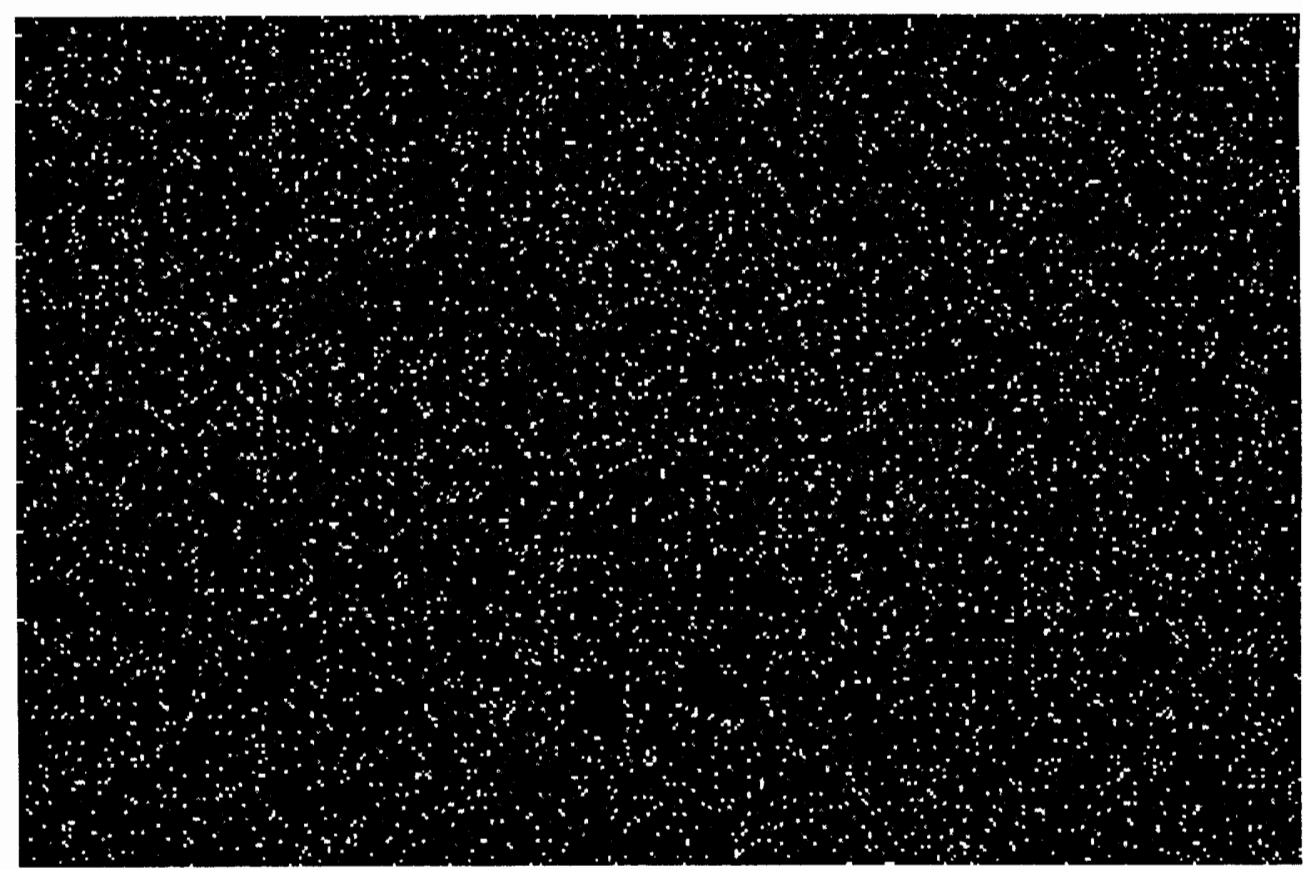

Figure 11: This figure shows a typical dark frame. The white specks are the 'hot pixels' which have a very high dark count. They are uniformly distributed over the frame. The dark frame used is a master dark frame combining 10 dark frames. All frames were taken at $20^{\circ} \mathrm{C}$ with a 20 second exposure time. 


\subsection{Exposure Time}

The dark count is linearly dependent on the exposure time, which means the dark count accumulates during the process of exposure (see Figure 12). In order to remove the correct amount of dark count, the effective dark-integration time of an exposure should be used instead of the 'real' exposure times. The real exposure time is only the length of time the shutter is open. In practice there is a lag between the end of flushing and the shutter opening and another lag between the time the shutter closes and the start of the read-out process. The dark-integration time is thus always longer than the actual exposure time. The difference is usually negligible with individual dark frames which have the same duration as the exposure, but it is essential to take this into account when using a master dark frame that is scaled. The software used supported the distinction between dark exposure time and real exposure time. It is therefore possible to use dark frames with different dark exposure times by scaling them with a constant [12]. 


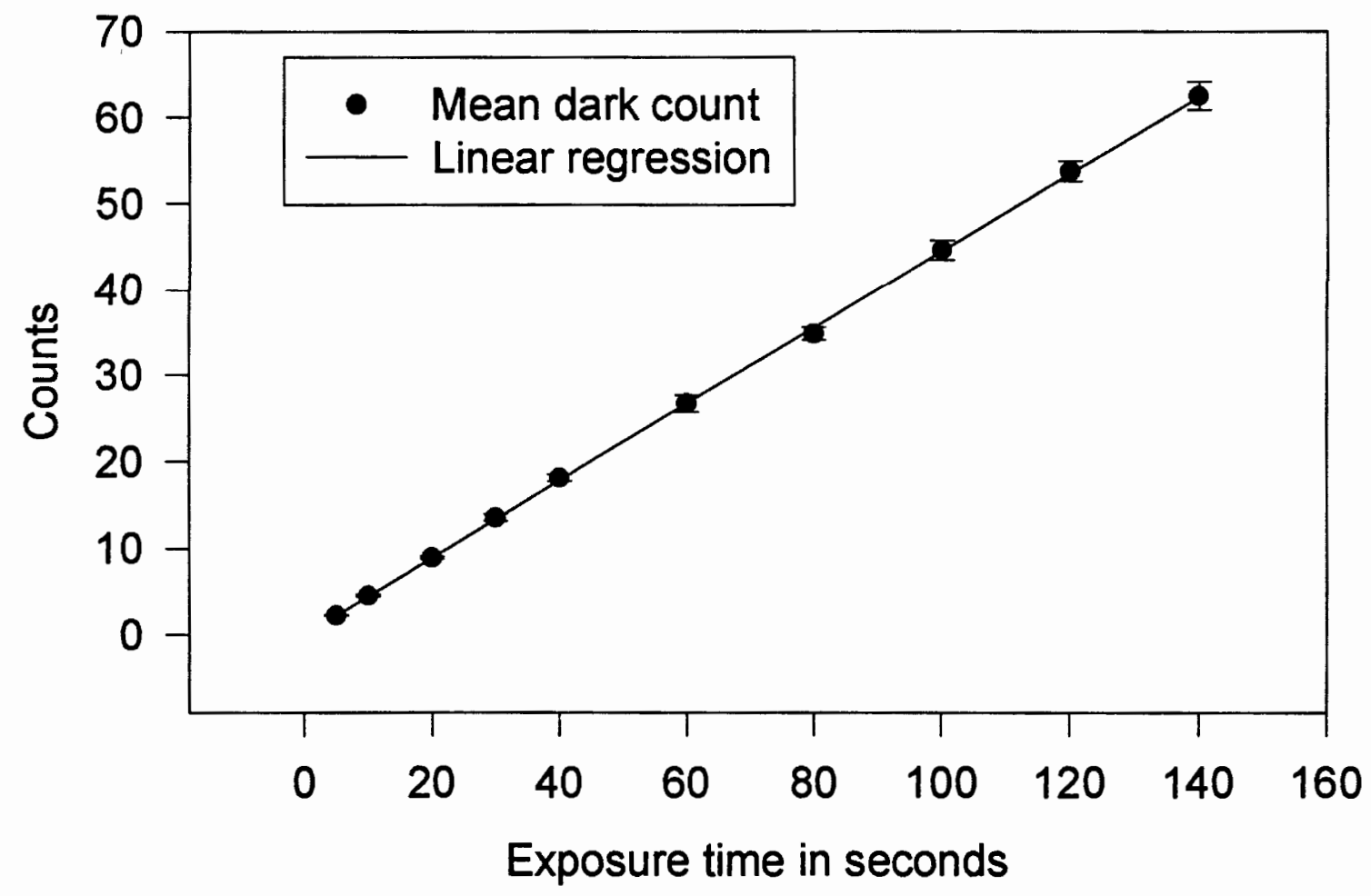

Figure 12: The exposure time dependence of the dark count is shown. For each exposure time, a mean dark count was calculated from 20 dark frames taken at $5^{\circ} \mathrm{C}$.

\subsection{Dark Count Populations}

The CCD camera used works in a multi-pinned-phase (MPP) mode. A feature of this mode is that there are multiple distinct populations, each with a well defined average dark count and with random fluctuations about the average. However, it is not possible to estimate a meaningful average dark count for all pixels[12]. In Figure 13 the dark count populations in a 100,000 pixel area are shown. 


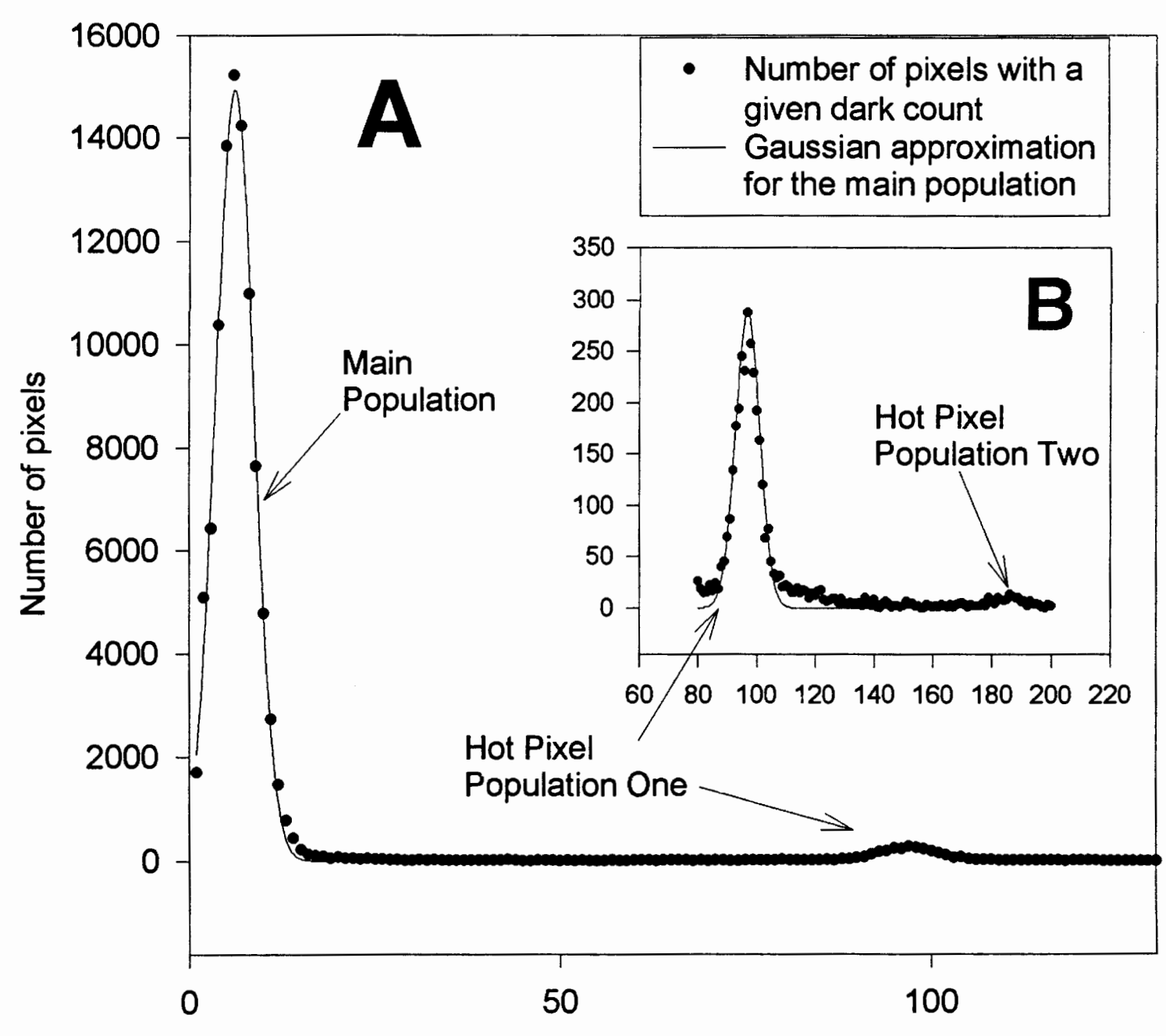

Dark count

Figure 13: Graph A shows the main population and hot pixel population one of the dark count at $5^{\circ} \mathrm{C}$ with a 20 second exposure time. The dark frame used was a master dark frame combining 10 dark frames. In Graph B the hot pixel population one and hot pixel population two from graph A are shown in an enlarged scale.

Around $95 \%$ of the pixels are in the main population and have an average dark count of 10 counts. The next population, denoted as hot pixel population one, already contains a 10 times larger dark count, or about 100 counts. This population 
makes up around $2 \%$ of the total amount of pixels. Hot pixel population two is only $0.1 \%$ of all pixels with an average dark count of 190 counts. The remaining $3 \%$ of the population are uniformly distributed up to a maximum of 1000 dark counts. In fact, one extraordinary hot pixel out of these 100,000 pixels had a very high dark count of over 7000 counts.

\subsection{Temperature}

Temperature has a big influence on the dark count. The dark count $(D)$ is exponentially dependent on the temperature $(T)$ (see Figure 14A). A very convenient and expressive form is to write the dependence as

$$
D=D_{0} \cdot 2^{\frac{T-T_{0}}{\Delta T}}
$$

if a reference dark count $\left(D_{0}\right)$ for a reference temperature $\left(T_{0}\right)$ is known (see Figure 14B). $\Delta T$ is called the doubling temperature, because the dark count doubles if the temperature is increased by this temperature[12]. By rearranging and taking the logarithm, Equation (44) changes to

$$
\ln \left(\frac{D}{D_{0}}\right)=\frac{\ln (2)}{\Delta T} \cdot\left(T-T_{0}\right)
$$


If we now plot $\ln \left(\frac{D}{D_{0}}\right)$ vs $T-T_{0}$ (see Figure 14B) we can determine the slope $(s)$ of the best fitting line by a linear regression and since

$$
s=\frac{\ln (2)}{\Delta T}
$$

we can then calculate the doubling temperature $\Delta T$. The result is $5.71^{\circ} \mathrm{C}$ with an $R^{2}$ value of 0.9998 , a nearly perfect fit by the linear regression. In the literature (see [12]) a doubling temperature around $6^{\circ} \mathrm{C}$ is mentioned for the CCD camera used.
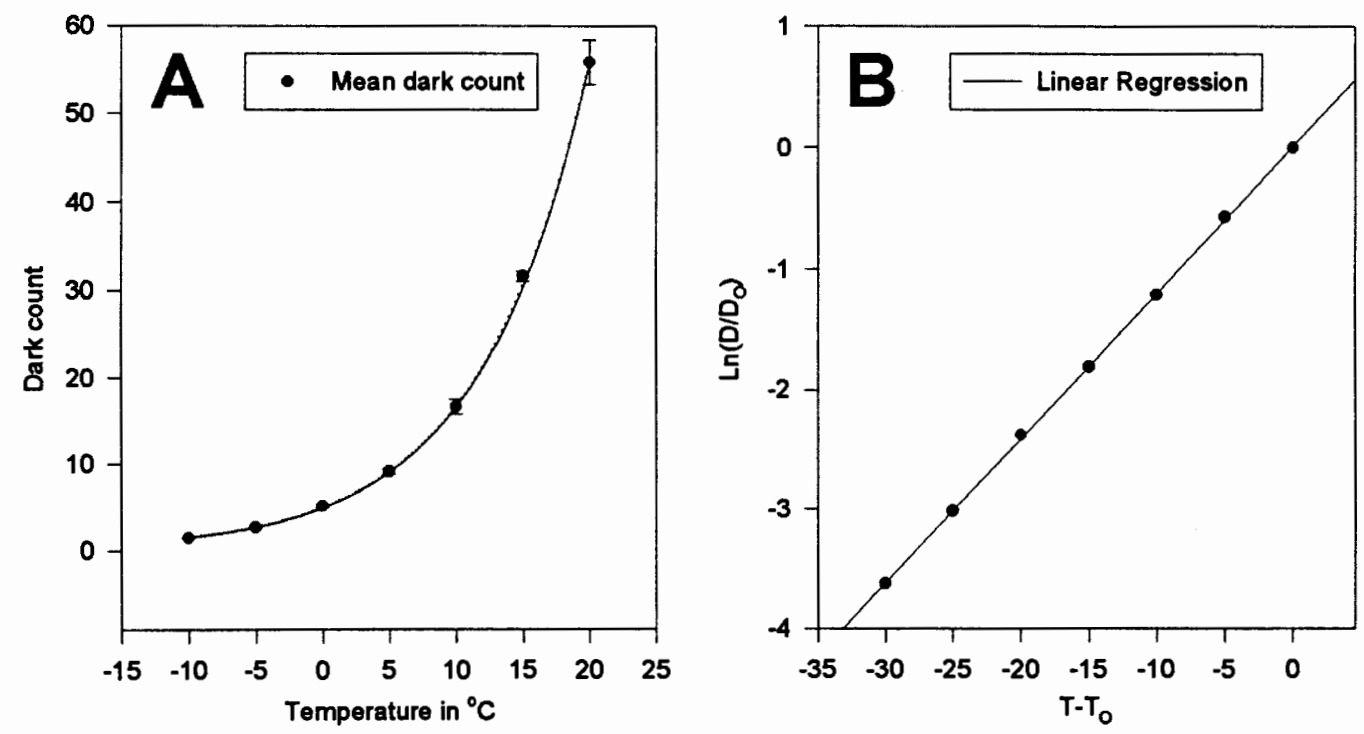

Figure 14: In Graph A the temperature $(T)$ dependence of the dark count $(D)$ is shown. In Graph B $\ln \left(\frac{D}{D_{0}}\right)$ vs $T-T_{0}$ is plotted with $T_{0}=20^{\circ} \mathrm{C}$ and $D_{0}=55.8$ counts. The doubling temperature $\Delta T$ can be determined by the slope of the plot. The dark frame exposure time was 20 seconds. 


\subsection{Dark Noise}

The dark count is Poisson count, which means that the noise of a dark count signal should be equal to the square root of the signal. Since the dark count is highly dependent on the temperature and the exposure time, the dark noise should also be dependent on them both.

\subsubsection{Temperature}

Figure 15A shows the temperature dependence of the dark noise. The dark noise is obtained by the subtraction method described in section 3.2.1.

The mean of the dark noise is squared and treated as a type of 'dark count'. Once this is done, it is possible to plug the squared dark noise in equation (45). Using linear regression we obtain a doubling temperature of $5.88^{\circ} \mathrm{C}$ with a $R^{2}$ value of 0.9979 for this artificial 'count' (see graph on the bottom in Figure 15). The close value of the doubling temperatures of the real dark count and the squared mean of the dark noise reinforces the assumption that the dark count can be treated as a Poisson count. 

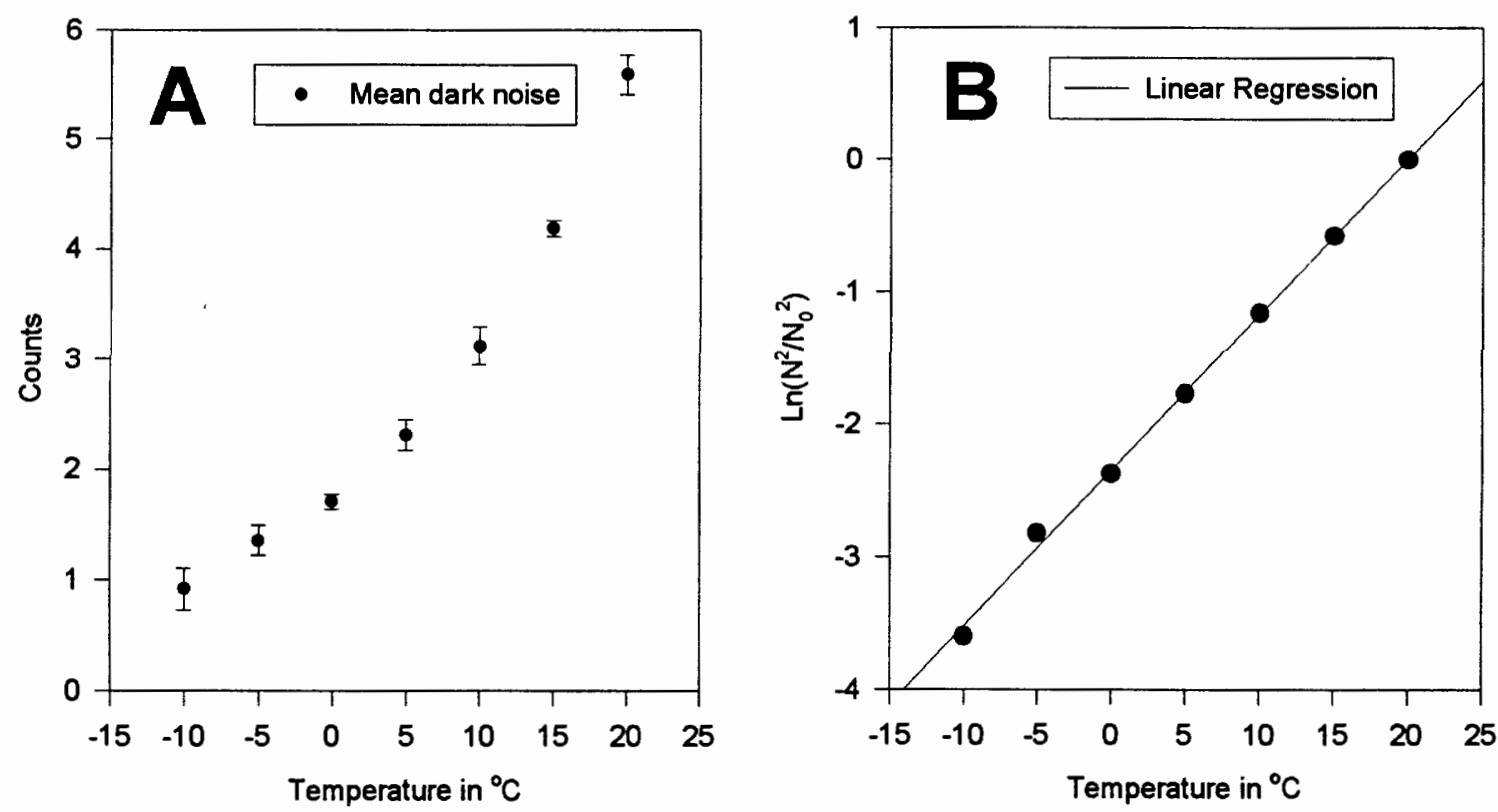

Figure 15: Graph A shows the temperature dependence of the dark noise. In Graph B $\ln \left(\frac{N^{2}}{N_{0}^{2}}\right)$ vs $T-T_{0}$ is plotted. It is possible to fit a straight line through the plot since the squared dark noise is equal to the dark count. The dark frame exposure time was 20 seconds.

\subsubsection{Exposure Time}

The exposure time dependence of the dark noise is shown in Figure 16. Comparison of the mean and the median dark count with the squared dark noise is shown in Figure 16B. In general, the dark count is very closely distributed around a mean value with a few runaway pixels (hot pixels). The median disregards these pixels, so the median is expected to be slightly smaller than the squared dark noise, as it can be seen in Figure 16B. The dark noise takes these pixels, as does the mean dark 
count, into account, but because the dark noise is only the square root of the dark count, these hot pixels have a minor influence on the dark noise compared to the influence on the mean dark count. As expected, the squared dark noise is between the mean and the median of the dark count and closer to the median (see Figure $16)$.

\subsection{Is it Possible to Use Dark Frames Taken at}

\section{Another Temperature ?}

The dark count for low temperatures is much smaller compared to that associated with high temperatures, therefore it is desirable to take images at low temperatures. However, dark frames taken at low temperatures have a small signal-to-noise ratio compared to dark frames taken at high temperatures. This can be seen in the following relationship (from the fact that $D$ increases exponentially with temperature):

$$
\left(\frac{S}{N}\right)_{d a r k c o u n t}=\frac{D}{N_{D}}=\frac{D}{\sqrt{D}}=\sqrt{D}
$$



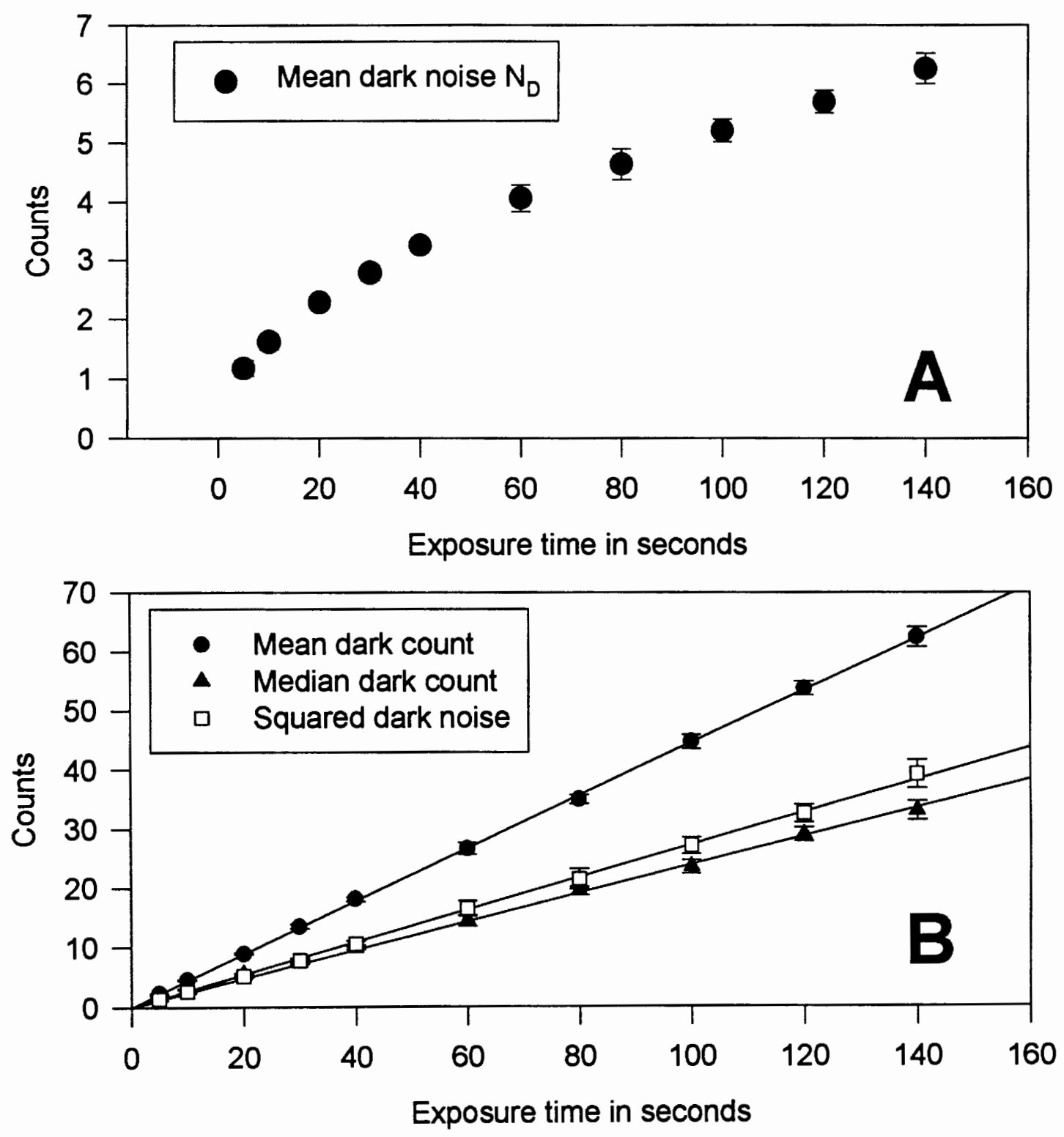

Figure 16: Graph A shows the exposure time dependence of the mean dark noise. In Graph B the exposure time dependence of the mean and median dark count is compared to the exposure time dependence of the squared dark noise. 
If a dark frame $\left(D_{1}\right)$ taken at a high temperature $\left(T_{1}\right)$ (and therefore a high $\left.\left(\frac{S}{N}\right)_{D 1, T 1}\right)$ could be scaled down to a lower temperature $\left(T_{2}\right)$, it would have a higher $\left(\frac{S}{N}\right)_{D 1, T 2}$ than the $\left(\frac{S}{N}\right)_{D 2, T 2}$ of a dark frame $\left(D_{2}\right)$ taken at temperature $T_{2}$ with the same exposure time. Let $D 1$ be $l$ times larger than $D 2$. Then we can compare the $\frac{S}{N}$ of the two dark frames for $T_{2}$

$$
\left(\frac{S}{N}\right)_{D 1, T 2}=\left(\frac{S}{N}\right)_{D 1, T 1}=\frac{D 1}{N_{D 1}}=\frac{l \cdot D 2}{\sqrt{l} \cdot N_{D 2}}=\sqrt{l} \cdot\left(\frac{S}{N}\right)_{D 2, T 2}
$$

A scaled down dark frame from $T_{1}$ to $T_{2}$ would have a $\sqrt{l}$ higher $\frac{S}{N}$ than a dark frame taken at $T_{2}$. Using Equation (44) we can calculate the scaling factor $l$ :

$$
l=2^{\frac{T_{1}-T_{2}}{\Delta T}}
$$

where $\Delta T$ is the doubling temperature. For the CCD camera ${ }^{1}$ used, the factor $l$ would be 20.8 for a temperature difference of $25^{\circ} \mathrm{C}$, therefore the $\frac{S}{N}$ would improve by a factor 4.56 .

In order to verify if a dark frame can be scaled down, dark frames at different temperatures were taken. The dark frames $\left(D_{1}\right)$ taken at $15^{\circ} \mathrm{C}$ were scaled down to $-10^{\circ} \mathrm{C}$ and then subtracted from dark frames $\left(D_{2}\right)$ taken at $-10^{\circ} \mathrm{C}$. The average dark count of the scaled down dark frames were, as expected, very close to the average of the dark frames taken at $-10^{\circ} \mathrm{C}$. If we define the difference frame as the difference

\footnotetext{
${ }^{1}$ The camera has a doubling temperature of $5.71^{\circ} \mathrm{C}$.
} 
between $D_{1}$ and $D_{2}$, the expected mean of the counts in all pixels is zero, and the noise in the difference frame $\left(N_{D i f f}\right)$ can be calculated by the following equation.

$$
\begin{aligned}
N_{D i f f} & =\sqrt{N_{1}^{2}+N_{2}^{2}} \\
& =\sqrt{N_{D 2, T 2}^{2}+2 \cdot N_{B, T 2}^{2}+\frac{N_{D 1, T 1}^{2}+2 \cdot N_{B, T 2}^{2}}{l^{2}}} \\
& =\sqrt{0.95^{2}+2 \cdot 5.61^{2}+\frac{4.18^{2}+2 \cdot 6.82^{2}}{20^{2}}} \\
& =8.01
\end{aligned}
$$

Surprisingly, the noise in the difference frame was found to be 9.1. This is higher than expected, but still less than the noise ${ }^{2}$ in a difference frame made out of two dark frames taken at $-10^{\circ} \mathrm{C}$. While at first glance this method appears to improve the signal to noise ratio, it does not take into account that some pixels are scaled improperly. In fact, it turned out that the maximum error ${ }^{3}$ increased from 60 to 1100, when a scaled down dark frame was used! What causes this unexpected increase in noise and this large maximum error? The dark count in the hot pixels is very poorly scaled down. On the average, the scaled down dark count was three times too small for the hot pixels. A further look at the hot pixels showed that the hot pixel populations have different doubling temperatures. With the linear regression

\footnotetext{
${ }^{2}$ This noise is around 11.3 counts.

${ }^{3}$ The maximal error is the largest absolute value in the difference image.
} 
done in Figure 17 the doubling temperature $\left(\Delta T_{h o t}\right)$ for hot pixel population one ${ }^{4}$ turned out to be $7.40^{\circ} \mathrm{C}$ with an $R^{2}$ value of 0.9997 using Equation (44), (45) and (46). Therefore, the main population could be scaled down accurately, but not the hot pixel populations at the same time. Due to this and the resulting large maximum error it is not possible to use a global doubling temperature for scaling down dark frames taken at another temperature.

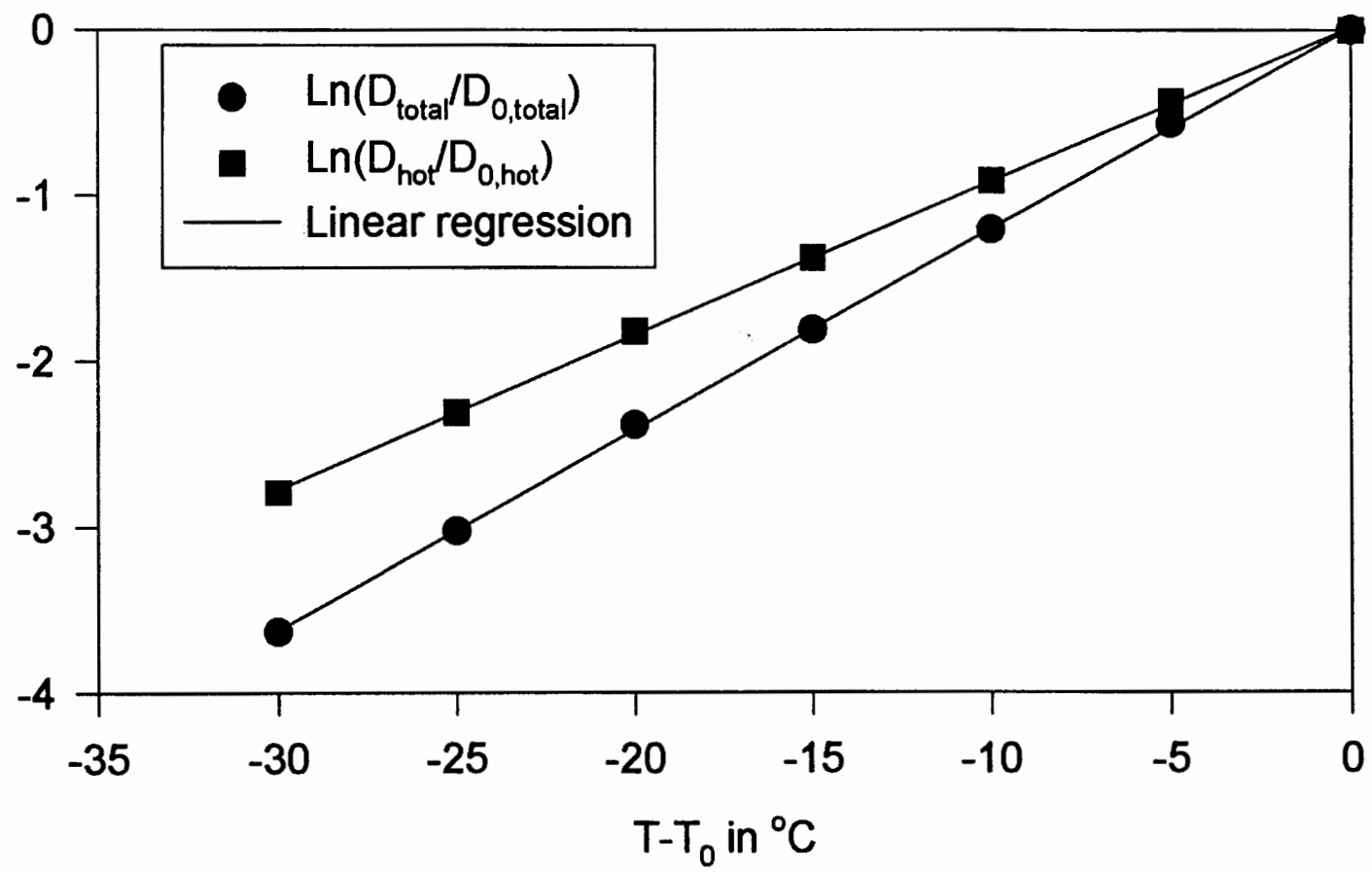

Figure 17: In this graph $\ln \left(\frac{D_{h o t}}{D_{0, h o t}}\right)$ versus $T-T_{0}$ is plotted where $D_{h o t}$ is the mean dark count of the hot pixel population, $D_{0, \text { hot }}=403.8$ counts and $T_{0}=20^{\circ} \mathrm{C}$. The doubling temperature $\Delta T_{\text {hot }}$ for the first hot pixel population can be determined using the slope of the plot. For comparison, the plot $\ln \left(\frac{D_{\text {total }}}{D_{0, \text { total }}}\right)$ vs $T-T_{0}$ is shown, where $D_{\text {total }}$ is the mean dark count of the total population and $D_{0, \text { total }}=55.7$ counts.

\footnotetext{
${ }^{4}$ Hot pixel population onecan be approximated with a Gaussian curve, see Figure 13, and the mean of these Gaussian curves were used for the calculations.
} 


\subsection{Effect of Past Images on the Dark and Bias}

\section{Count}

Another important aspect is to determine whether or not the dark and bias count is dependent on past images. If flat-field frames, which are frames taken from a uniform light source, are taken before dark and bias frames, a large increase of the dark and bias counts can be observed. In Figure 18 the dark and bias count is shown for different sets of frames:

- set 1-5 : each set consists of 1 dark $^{5}$ and 1 bias frame.

- set 6-10: each set consists of 1 flat-field ${ }^{6}, 1$ dark and 1 bias frame.

- set 11-25: each set consists of 1 dark and 1 bias frame.

It can be observed, that the dark and bias count is stable for the first 5 sets, but for set 6-10 a large increase of the dark count and bias count is caused by the flat-field frames taken before. For set 11-25 an exponential decrease of the dark and bias count can be observed until the count drops to the values seen in the first five sets.

In order to verify if the incoming light is the source of this increase, an experiment was done in which only a part of the chip was illuminated by light ${ }^{7}$. In Figure 19

\footnotetext{
${ }^{5}$ The dark exposure time was 1 second.

${ }^{6}$ The flat-field exposure time was 20 seconds, the frame was not saturated.

${ }^{7}$ An image was taken from a small, bright laser spot, exposure time 0.15 seconds.
} 

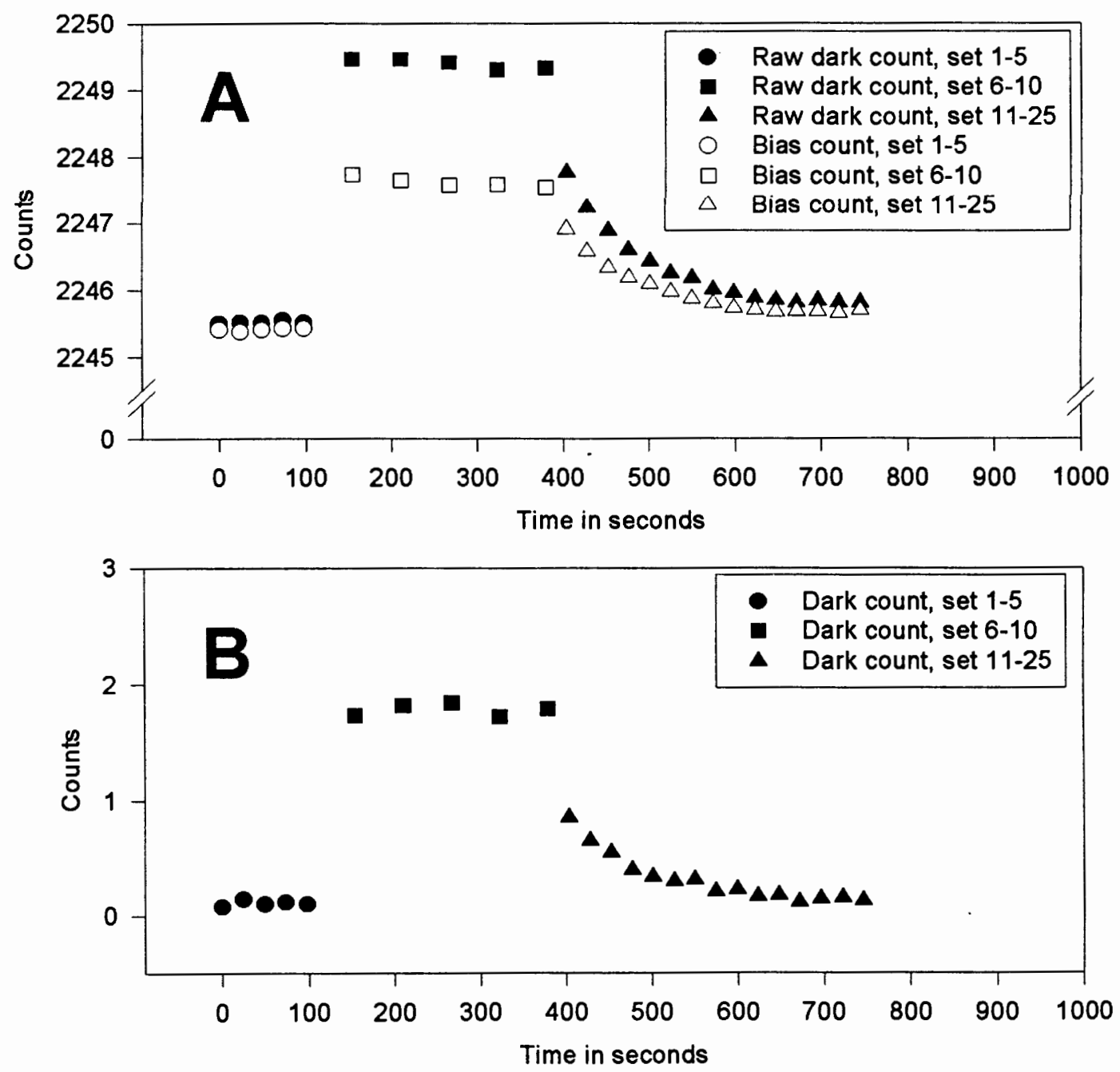

Figure 18: In Graph A, the mean raw dark count and mean bias count for the indicated sets of frames is shown. Graph B shows the dark count (raw dark count minus bias count) for each set. All frames were taken at $-10^{\circ} \mathrm{C}$. The exposure times were 1 seconds for the dark frame and 20 seconds for the flat-field frame. The short exposure time for the dark frame was chosen in order to be able to collect a sufficient amount of data in the short time after the flat-field frames were taken. An increase of the counts can be seen for sets in which a flat-field frame was taken before the dark and bias frames (set 6-10). 
the time dependence of the average dark count for two different areas of the chip is shown: One area was illuminated by the image taken before and the other one was not illuminated. A change in the dark count could only be found in the area that had been illuminated. By exaggerating the exposure time for the light image ${ }^{8}$ a white spot in the dark frame, immediately taken after the image, can be seen (See Figure 20).

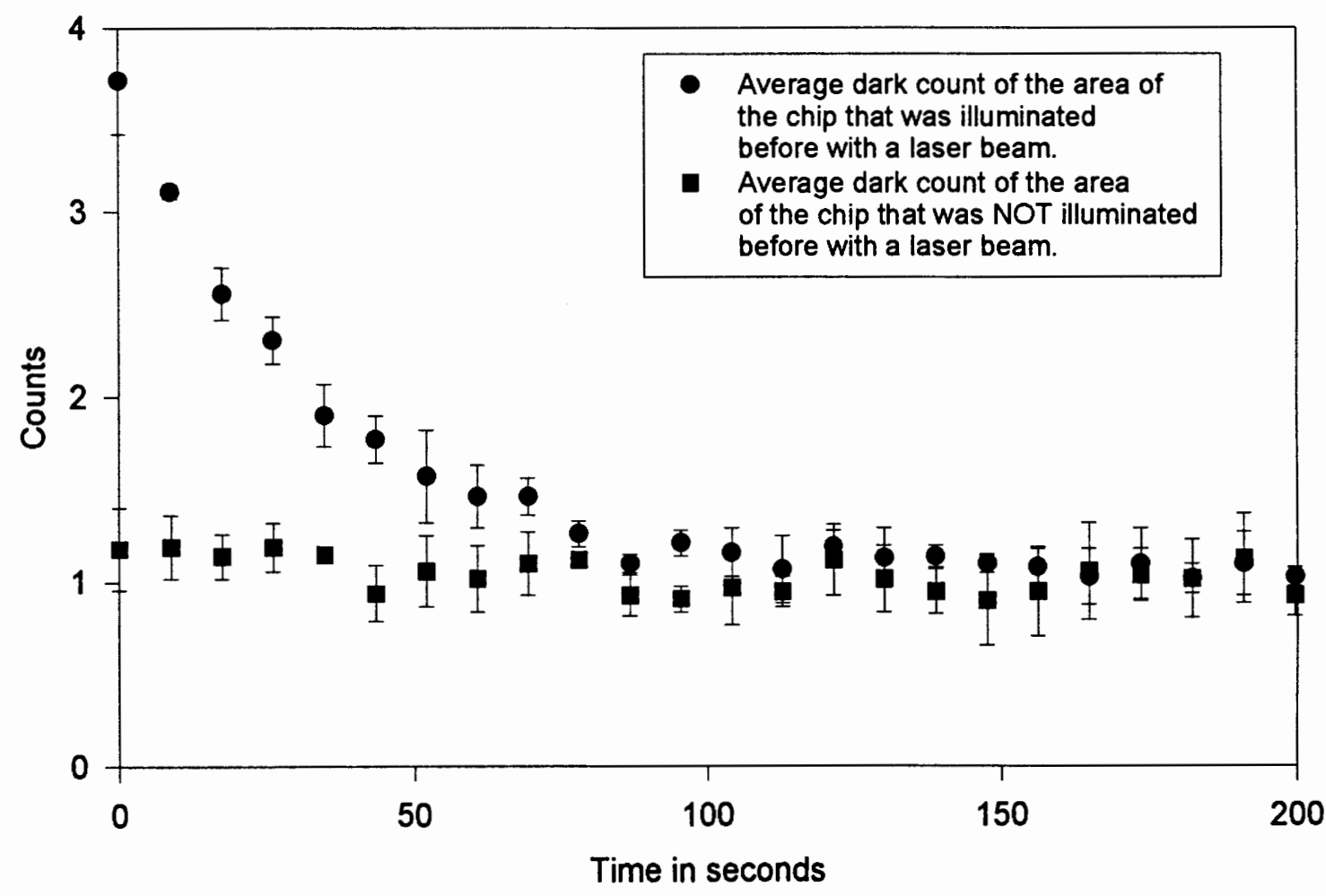

Figure 19: This figure shows the dark offset for two different areas of the chip: One area was illuminated before by taking an image of a bright laser spot. The other area was not illuminated. All frames were taken at $5^{\circ} \mathrm{C}$. The image exposure time was 0.15 seconds and the dark exposure time was 3 seconds.

\footnotetext{
${ }^{8}$ Exposure time 5 seconds instead of 0.15 seconds.
} 

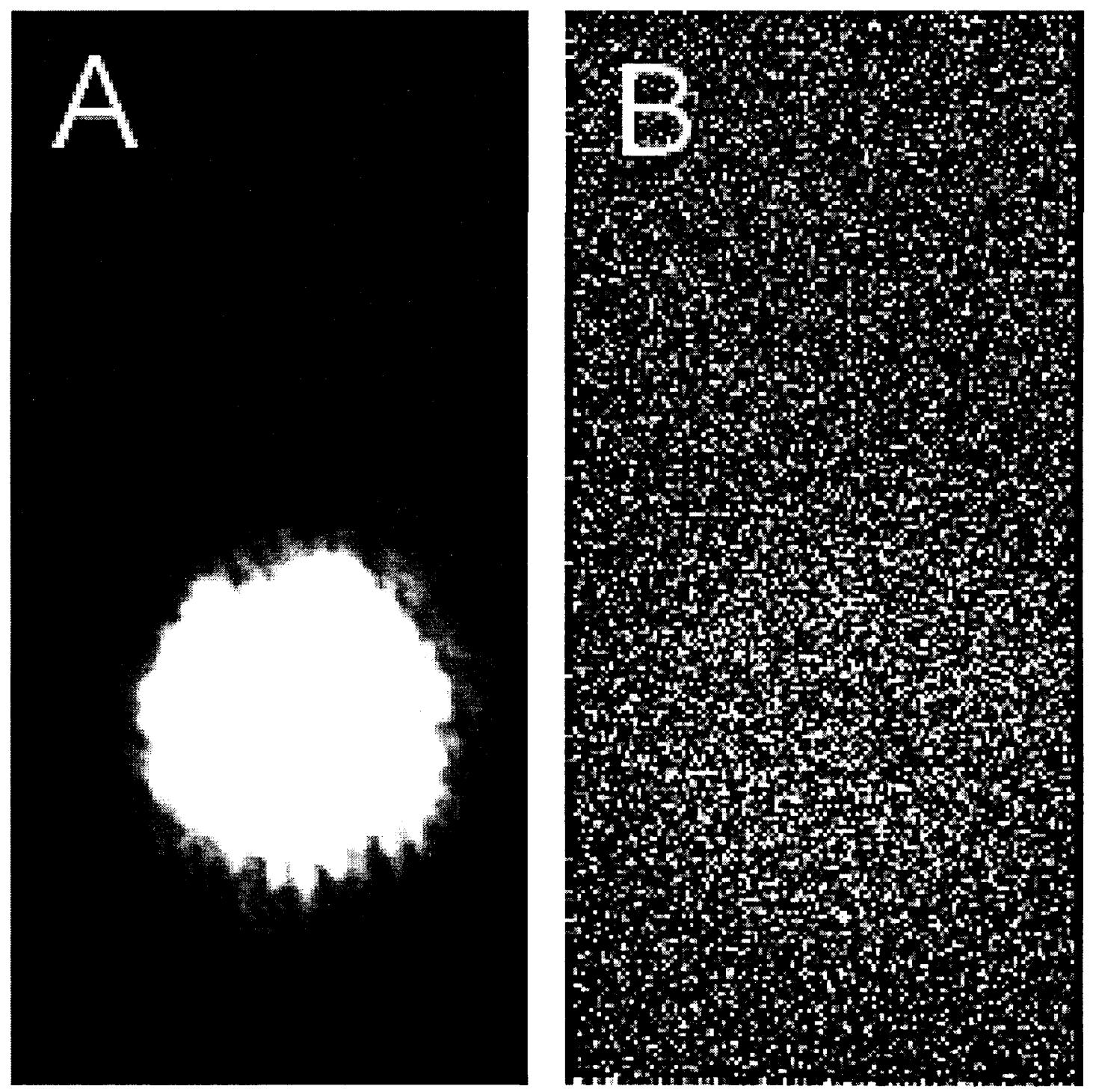

Figure 20: This Figure shows the effect of increased dark count caused by exposure of the chip to light. Graph A shows the image of a laser spot on a video screen. Graph B displays a dark frame taken immediately after the image of the laser spot was taken. The frames were taken at $5^{\circ} \mathrm{C}$. The image exposure time was 5 second and the dark exposure time was 3 second. 
How can the incoming light cause this increase in the dark and bias count? One possible source is an increase of the temperature on the surface of the chip. But two reasons contradict this assumption.

- As Figure 18B shows, the dark count increased by a factor of 15 , if a flat-field frame is taken before the dark frame. This would correspond to an unreasonable temperature increase of at least $20^{\circ} \mathrm{C}$ since the doubling temperature is $5.71^{\circ} \mathrm{C}$. Similar results were obtained in the temperature range of $-15^{\circ} \mathrm{C}$ to $20^{\circ} \mathrm{C}$.

- The average dark count increased by the factor of 15 , but the dark count of the hot pixel increased only slightly. Therefore, the effect is not a multiplicative effect, it is an additive effect. If a temperature increase would be the reason for the larger dark count, then the effect would be multiplicative and the hot pixels would increase at least by the same factor since the doubling temperature $\Delta T_{\text {hot }}$ is larger than the average doubling temperature $\Delta T$.

A more realistic explanation for this additive effect is a kind of 'storage' of the light count with a slow, time-dependent release. I denote this offset as 'dark offset'. In Figure 21 the time dependence of the dark offset is shown for different flat-field exposure times. After approximately 50 second flat-field exposure time, the dark 
offset becomes saturated. Note that it is possible to fit an exponential curve over each set of decaying dark offset (see Figure 21). The halftime $\Delta t$ is $50 \pm 1.7$ seconds.
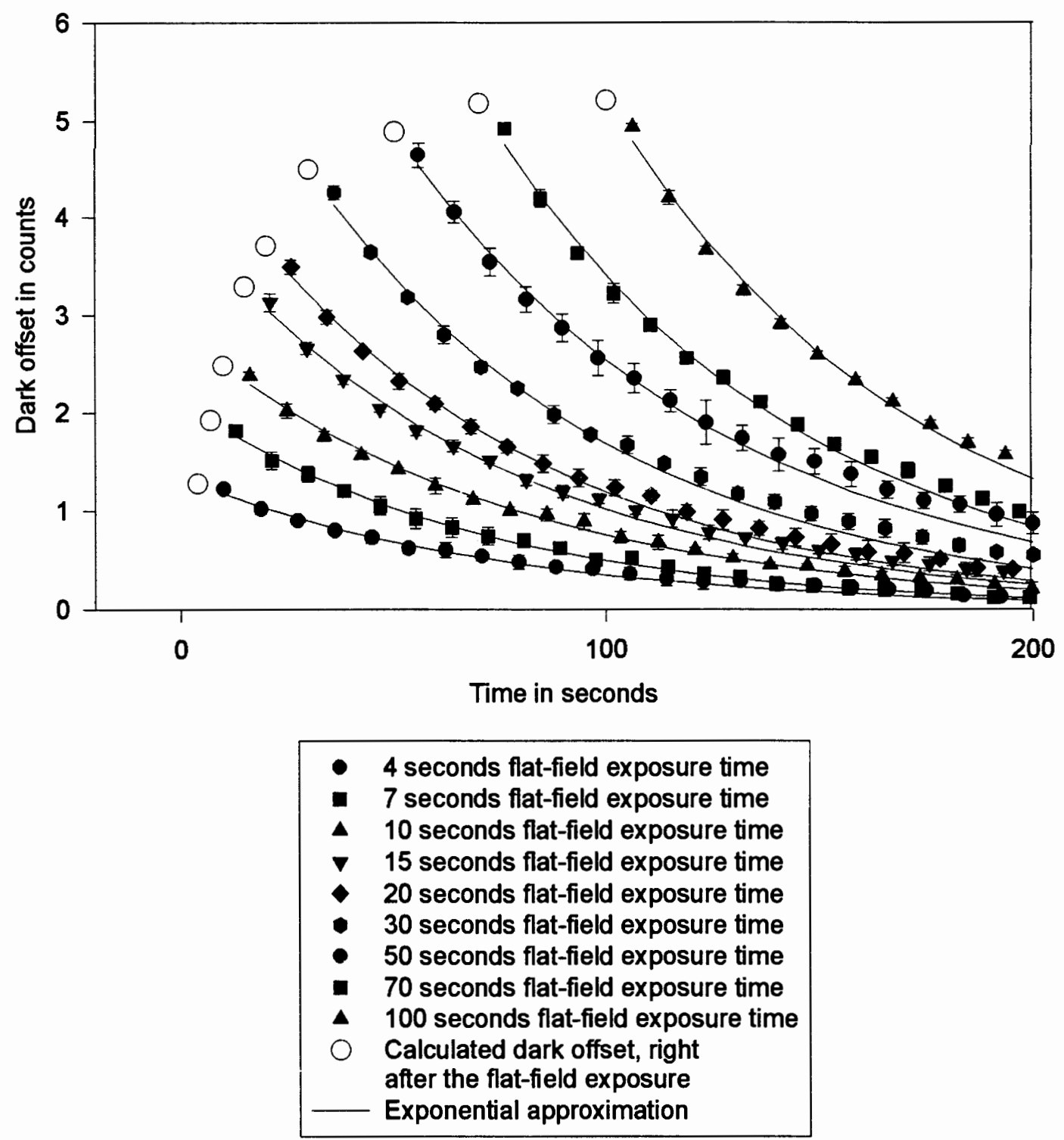

Figure 21: The decay of the dark offset after different flat-field exposure times as a function of time is shown in this figure. Open circles denote the calculated dark offset at the end of the flat-field exposure. Flat-field exposure starts at $t=0$. Closed symbols indicate the decay of the dark offset with time, where time is the time since the beginning of the flat-field exposure. 


\section{Chapter 6}

\section{Flat-Field Frames}

The last frame needed to calibrate an image is the flat-field frame. Flat-field calibration corrects for irregularities across the telescope's field of view and variations in the sensitivity of the CCD itself, i.e., a flat-field frame is a map of the relative sensitivity of each pixel in the detector (see Equation (1)). They are created by imaging a uniformly illuminated surface. A typical flat-field frame is shown in Figure 22[14].

The flat-field count in a randomly picked pixel can functionally be separated in a light signal count $(F)$, a dark count $(D)$ and a bias count $(B)$.

$$
\begin{aligned}
F_{\text {raw }} & =(F+D+B)_{\text {raw }} \\
N_{F, \text { raw }}^{2} & =N_{F}^{2}+N_{D}^{2}+N_{B}^{2}
\end{aligned}
$$


In order to calibrate the flat-field frame, we have to remove the dark count $(\hat{D})$ and the bias count $(\hat{B})$. Since there is noise in the dark and in the bias count, the subtraction causes again an increase in the noise level. The corresponding signal and noise levels can be determined:

$$
\begin{aligned}
F_{c a l} & =(F+D+B)_{r a w}-\hat{D}-\hat{B} \\
N_{F, c a l}^{2} & =N_{F}^{2}+N_{D}^{2}+N_{B}^{2}+N_{\hat{D}}^{2}+N_{\hat{B}}^{2}
\end{aligned}
$$

Dividing by a flat-field frame should not change the average pixel value. Therefore it is advantageous to normalize the flat-field frame to an average pixel value of 1 count. This can be done by dividing the flat field frame by the average flat-field count of the flat field frame, denoted as $\bar{F}$. After normalizing the flat-field frame to an average value of 1 , the signal and noise levels are

$$
\begin{aligned}
S_{F, n r m} & =\frac{F_{c a l}}{\bar{F}} \\
N_{F, n r m}^{2} & =\frac{N_{F, c a l}^{2}}{\bar{F}^{2}}
\end{aligned}
$$

The signal-to-noise ratio stays unchanged since dividing or multiplying by a constant influences the signal and the noise the same way (see section 2.4.5).

$$
\frac{S_{F, n r m}}{N_{F, n r m}}=\frac{F_{c a l}}{N_{F, c a l}}
$$




\subsection{When is it Necessary to Take a New Flat-}

\section{Field Frame}

Since the flat-field correction is repeatable, it is essential to avoid introducing nonrepeatable patterns, such as from mechanical and optical effects, into the flat-field frame. The alignment between a CCD camera and the optical system must remain fixed for all sky images and flat-field frames, otherwise the pattern changes and a new correction has to be generated. The following mechanical considerations are critical to obtain a reliable flat-field correction[14].

- The camera-to-telescope alignment must not change as the system is pointed to different parts of the sky[14].

- A filter wheel must repeat its positioning very accurately so that shadows from dust on the filters will land on the same pixels. In a converging optical beam, as the filter is moved farther from the CCD, dust shadows enlarge and soften. At the same time, however, it becomes more difficult to accurately repeat a filter's position[14].

- Since it is impossible to remove a camera or filter wheel from a telescope and put it back in exactly the same orientation, a new flat-field frame must be obtained each time the equipment is adjusted[14]. 
- Telescope optics and the CCD's sensitivity may change according to the wavelengths of light. So it is essential that the flat-field frames are exposed to the same color as the sky images[14].

- A CCD's quantum efficiency varies with temperature. Therefore a new flatfield frame should be taken if the temperature changes[14].

\subsection{Flat-Field Targets}

In general, a distinction is made between three different flat-field targets.

Twilight Flat-Field: Twilight flat-fielding uses the twilight sky as the uniformly illuminated surface. There are two major problems which have to be considered. One is that the brightness of the twilight sky changes very fast. This makes it essential to normalize the flat-field frames for generating a master flatfield frame. The other problem is that the blue twilight sky matches poorly the typical reddish sky background on a moonless sky. This can cause problems since the pixels have different quantum efficiencies for different wavelengths. Furthermore, it is advantageous to generate a master flat-field frame due to the fact that there are stars visible even in the twilight sky[14]. 
Sky Flat-Field: Many different celestial images are combined into a master flatfield frame. The big advantage of this method is, that obviously the flatfield frame represents very well the wavelength distribution in the sky image. However, a huge number of celestial images are needed in order to process a master flat-field frame with a high signal-to-noise ratio[14].

Dome Flat-Field: A screen in front of the telescope, uniformly illuminated by lamps, is used as the target. Additionally, the light can be diffused by putting a white paper directly in front of the telescope's aperture. Dome flat-field frames have two advantages over twilight ones: The level of illumination can be controled and the wavelength of the light used can be matched to the color of the sky[14]. 

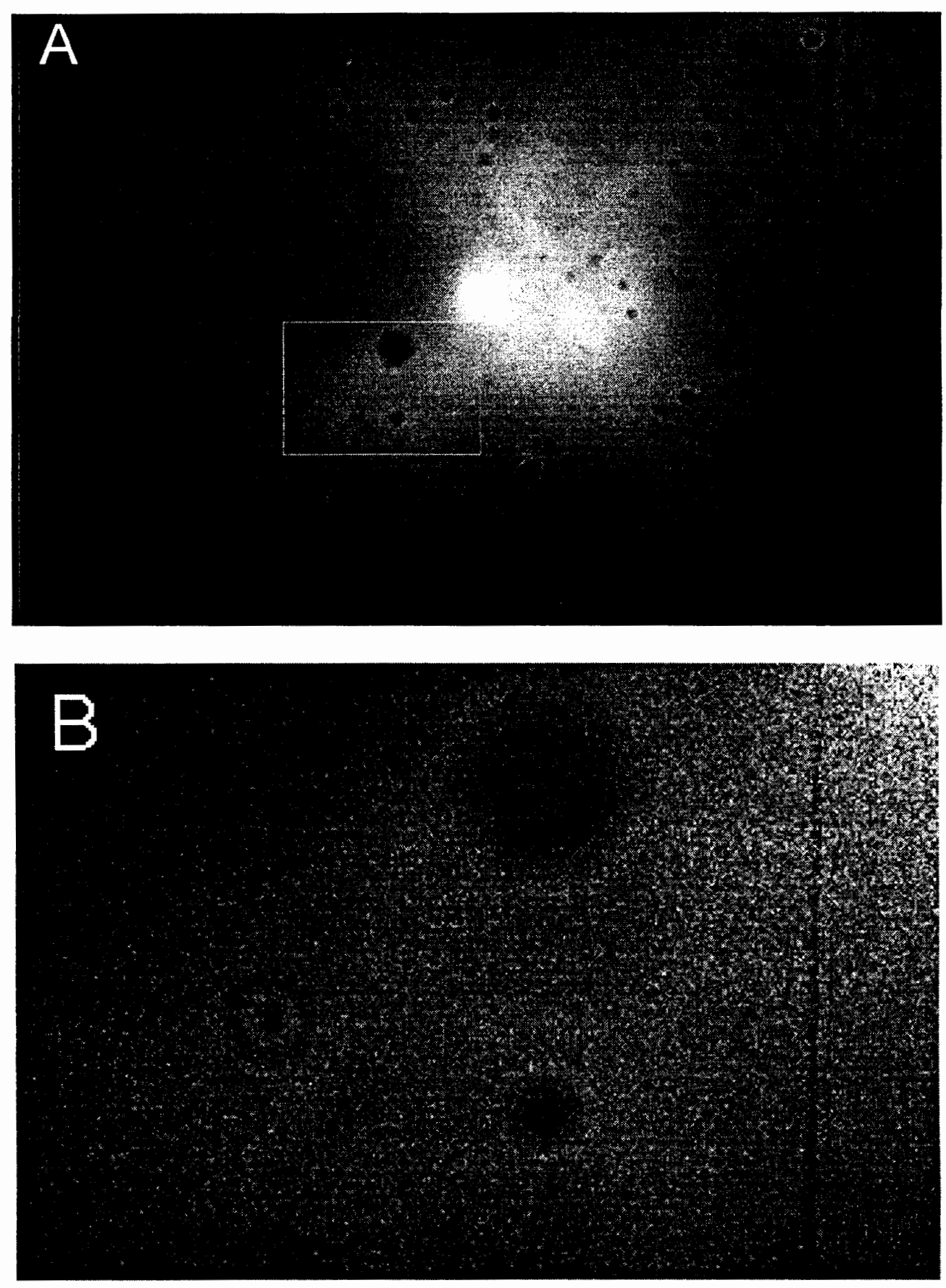

Figure 22: Graph A shows a typical flat-field frame. The dust shadows can be clearly recognized in Graph B, which shows the marked area in Graph A on an enlarged scale. The frame is taken at $20^{\circ} \mathrm{C}$ with a 20 second exposure time. 


\section{Chapter 7}

\section{Master Frames}

One way to obtain a high signal-to-noise ratio is to generate a master frame. A master frame is, in general, the average frame of frames with equal signal levels as explained in section 2.4.6. If we combine images with similar signal and noise levels, we can write

$$
\bar{N} \approx \frac{N_{i}}{\sqrt{n}} \quad \frac{\bar{S}}{\bar{N}} \approx \sqrt{n} \frac{S_{i}}{N_{i}}
$$

where $\bar{S}$ and $\bar{N}$ are the signal and noise level of the master frame and $S_{i}$ and $N_{i}$ are the signal and noise level of one of the original images. The noise in a master frame combining $n$ images is decreased by the factor $\sqrt{n}$ compared to the noise in a single frame. 


\subsection{Generating a Master Bias Frame}

Typically the bias level drifts up and down with time as the camera's electronics changes temperature. Thus, a bias frame taken immediately after an image gives the best estimate of the bias count in the image. Depending on how much a bias frame is drifting, it is sometimes advantageous to split the raw bias count $\left(B_{\text {raw }}\right)$ into a noise-free bias offset ${ }^{1}\left(B_{o f f s e t}\right)$ and a bias structure $\left(B_{\text {struc }}\right)$, that includes noise.

$$
B_{\text {raw }}=B_{o f f s e t}+B_{s t r u c}
$$

Therefore, if the raw bias count drifts with time, the bias offset can be calculated for an individual bias frame and then be subtracted to obtain the bias structure frame. A master bias frame can then be generated with the bias structure frame. Note that the bias offset is no longer included in the master bias frame. Thus, if the bias count has to be removed in an image, it is then essential not only to subtract the master bias frame, but to subtract the bias offset as well. A bias frame taken directly after the image can be used to calculate the bias offset and the master bias frame is used to subtract the bias structure. Henceforth, whenever we refer to a master bias frame, a decision on whether it is appropriate to split the bias count

\footnotetext{
${ }^{1}$ The bias offset is denoted as the average of the bias count over the whole frame or over a sufficiently large subframe.
} 
into a bias offset and a bias structure will have to be made. Using Equation (58) the noise in a master bias frame can be determined:

$$
N_{B, \operatorname{master}(n)}^{2}=\frac{N_{B}^{2}}{n}
$$

In Figure 23 the noise of a master bias frame generated by combining different numbers of bias frames is shown. Since the bias offset is noise free, the noise in a master bias frame is independent of whether the master frame was generated by combining bias structure frames or by combining raw bias frames.

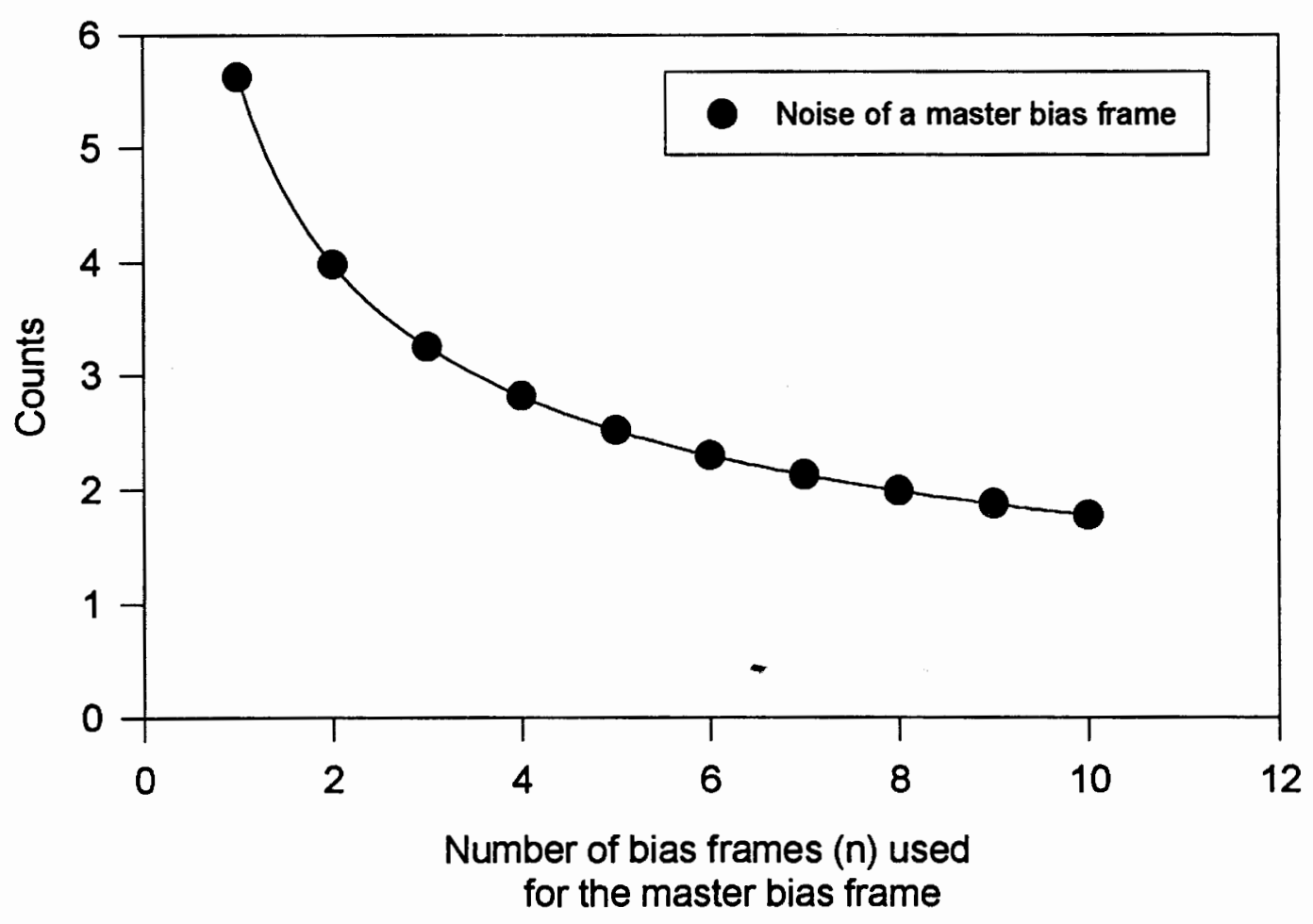

Figure 23: This figure displays the noise of master bias frames. The bias frames are taken at $-10^{\circ} \mathrm{C}$. 


\subsection{Generating a Master Dark Frame}

A dark frame needs to be calibrated as mentioned before (see chapter 5). In order to get a high signal-to-noise ratio, a master dark frame can be generated. In general, there are a couple of different methods that can be followed to process this master dark frame. Let us consider as an example two different methods of generating a master dark frame (see Table 2). Suppose we have a pool of 10 sets of frames, each set consisting of one dark frame and one bias frame.

Method One: We generate first a master bias combining the 10 bias frames ${ }^{2}$. The next step is to subtract the master bias frame from the raw dark frame. As the last step, themaster dark frames are processed with $n$ out of the 10 calibrated dark frames. It is important to point out, that the noise caused by the subtraction of the master bias frame of each individual dark frame does not decrease with the number of frames $n$ used for the master dark frame. This is because each dark frame is calibrated with the same master bias frame and therefore the noise in each dark frame corresponding to the subtraction of the master bias frame is not independent anymore.

Method Two: The first step in this method is to subtract each of the raw bias frames from the corresponding raw dark frames. Then the master dark frames

\footnotetext{
${ }^{2}$ Depending on the camera used, the bias count should be separated into a bias offset and a bias structure.
} 
are generated by using $n$ out of these 10 calibrated dark frames. Since each dark frame is calibrated with a different bias frame, the noise corresponding to these subtractions is independent from each other. Therefore the total noise of the master dark frame decreases with the number $n$ of calibrated dark frames used.

Method one has, as can be seen in Table 2, a better noise level than method two, if there are only a few calibrated dark frames used for the master dark frame. But since the noise caused by subtracting the master bias frame is not decreasing by increasing the numbers of combined dark frames, the noise level for 10 combined calibrated dark frames is equal for both methods. At first glance it looks like method one is superior to method two. However, since the noise level of the 'end result', the master dark frame, is equal for both methods and method two is much faster, the use of method two can be recommended. A graphical comparison of the noise level of the two methods is shown in Figure 24. It can be seen that when $n$ is 10 both methods result in essentially the same noise level. 


\begin{tabular}{|c|c|}
\hline Method one: & Method two: \\
\hline $\begin{array}{l}\text { A master bias is processed with the } \\
10 \text { bias frames. } \\
B_{\text {master(10) }}=\frac{1}{n} \cdot \sum_{i=1}^{10} B_{i} \\
N_{B, \text { master }(10)}^{2}=\frac{N_{B}^{2}}{10}\end{array}$ & \\
\hline $\begin{array}{l}\text { From each raw dark frame the master } \\
\text { bias frame is subtracted. } \\
D_{\text {cal1 }}=(D+B)_{\text {raw }}-B_{\text {master }(10)} \\
N_{D, \text { cal1 }}^{2}=N_{D}^{2}+N_{B}^{2}+\frac{N_{B}^{2}}{10}\end{array}$ & $\begin{array}{l}\text { From each raw dark frame one single } \\
\text { bias frame is subtracted. } \\
D_{\text {cal2 }}=(D+B)_{\text {raw }}-B \\
N_{D, \text { cal2 }}^{2}=N_{D}^{2}+N_{B}^{2}+N_{B}^{2}\end{array}$ \\
\hline $\begin{array}{l}\text { Master dark frame with } n \text { out of the } \\
\text { ten } D_{\text {cal }} \\
D_{\text {cal } 1, \text { master }(n)}=\frac{1}{n} \cdot \sum_{i=1}^{n} D_{\text {cal1 }} \\
N_{D, \text { cal } 1, \text { master }(n)}^{2}=\frac{N_{D}^{2}}{n}+\frac{N_{B}^{2}}{n}+\frac{N_{B}^{2}}{10}\end{array}$ & $\begin{array}{l}\text { Master dark frame with } n \text { out of the } \\
\text { ten } D_{\text {cal }} \\
D_{\text {cal } 2, \text { master }(n)}=\frac{1}{n} \cdot \sum_{i=1}^{n} D_{c a l 2} \\
N_{D, \text { cal } 2, \text { master }(n)}^{2}=\frac{N_{D}^{2}}{n}+\frac{N_{B}^{2}}{n}+\frac{N_{B}^{2}}{n}\end{array}$ \\
\hline
\end{tabular}

Table 2: Summary of two ways to generate a master dark frame. 


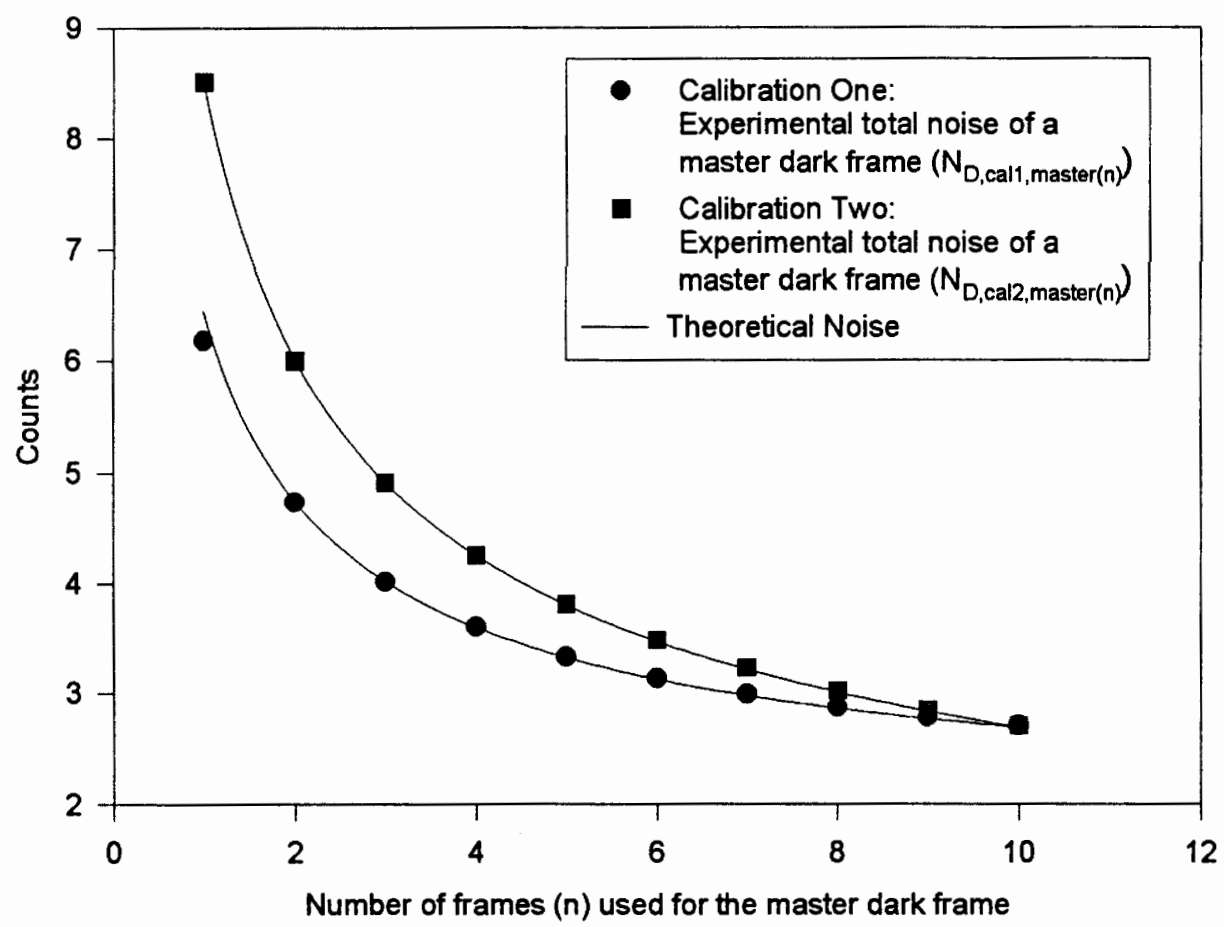

Figure 24: Comparison of the two methods for master dark frame calibration. All frames are taken at $15^{\circ} \mathrm{C}$. The dark frame exposure time was 10 seconds.

\subsection{Generating a Master Flat-Field Frame}

Just as the dark frame, the flat-field frame needs to be calibrated as well (see chapter 6). A master flat-field frame can be generated since it is advantageous for the signalto-noise ratio. There are different methods of calibrating a master flat-field frame. Two methods will be discussed. Once again we have a pool of 10 sets of (flat-field 
frame, dark frame, and bias frame). Table 3 shows an overview of the signal and noise levels for both calibration methods.

Method One: First, a master bias frame is generated with the 10 bias frames. The 10 dark frames are calibrated with this master bias frame and then combined into a master dark frame. The next step is to subtract the master bias frame and the master dark frame from each flat-field frame. One important thing to mention is, that the noise caused by the subtraction of the master bias frame from the flat-field frame and the noise caused by the subtraction of the master bias frame from each dark frame cancels out. The reason for this is, the same master bias frame is subtracted from the dark frame and from the flat-field frame. With some algebra it can be shown that these two operations cancel each other out, as shown below.

$$
\begin{aligned}
F_{\text {cal1 }} & =F_{\text {raw }}-D_{\text {master(10) }}-B_{\text {master (10) }} \\
& =F_{\text {raw }}-\frac{1}{10} \cdot \sum_{i=1}^{10}\left(D_{\text {raw }}-B_{\text {master (10) }}\right)-B_{\text {master (10) }} \\
& =F_{\text {raw }}-\frac{1}{10} \cdot \sum_{i=1}^{10}\left(D_{\text {raw }}\right)+\frac{1}{10} \cdot 10 \cdot B_{\text {master (10) }}-B_{\text {master }(10)} \\
& =F_{\text {raw }}-\frac{1}{10} \cdot \sum_{i=1}^{10}\left(D_{\text {raw }}\right)
\end{aligned}
$$

It can be observed that the bias frame drops out completely. The last step is then to generate the master flat-field frame with $n$ out of the 10 calibrated 
flat-field frames. Similar as method one in the master dark frame calibration, the noise corresponding to the subtraction of the master dark frame and the master bias frame is not decreasing with an increasing number of flat-field frames used for the master flat-field frame.

Method Two: In this method, the first step is to subtract the raw dark frame from the corresponding flat-field frame. The master flat-field frame is then generated by $n$ out of the 10 calibrated flat-field frames. The noise of the master flat-field frame decreases proportional to $\sqrt{n}$, since all sources of noise are independent from each other.

Similar to the master dark frame calibration, method one has a better noise level for a single flat-field frame; but the noise level for a master flat field frame generated out of all the 10 flat-field frames is equal for both methods. Since method two is superior in processing speed, memory requirements and imaging time, the use of this method can be recommended to easily obtain a flat-field frame with a high signal-to-noise ratio. The difference of the squared noise of the two master flat-field frame calibration methods is shown in Figure 25. 


\begin{tabular}{|c|c|}
\hline Method one: & Method two: \\
\hline $\begin{array}{l}\text { A master bias and a master dark is } \\
\text { processed with the } 10 \text { bias frames and } \\
\text { the } 10 \text { dark frames. } \\
B_{\text {master }(10)}=\frac{1}{10} \cdot \sum_{i=1}^{10} B_{i} \\
D_{\text {cal }}=D_{\text {raw }}-B_{\text {master }(10)} \\
D_{\text {master }(10)}=\frac{1}{10} \cdot \sum_{i=1}^{10} D_{\text {cal }}\end{array}$ & \\
\hline $\begin{array}{l}\text { Subtract master bias and master dark } \\
\text { from each raw flat-field frame. } \\
\begin{aligned} F_{\text {cal1 }}= & (F+D+B)_{\text {raw }} \\
& -D_{\text {master }(10)-}-B_{\text {master }(10)}\end{aligned} \\
\begin{aligned} N_{F, \text { cal1 }}^{2}= & N_{F}^{2}+N_{D}^{2}+N_{B}^{2} T^{0} \\
& +\frac{N_{B}^{2}}{10}+\frac{N_{B}^{2}}{10}+\frac{N_{F}^{2}}{6}+\frac{N \pi^{0}}{0}\end{aligned}\end{array}$ & $\begin{array}{l}\text { Subtract one single raw dark frame } \\
\text { from each raw flat-field frame } \\
\begin{aligned} F_{\text {cal } 2}= & (F+D+B)_{\text {raw }} \\
& -(D+B)_{\text {raw }}\end{aligned} \\
\begin{aligned} N_{F, \text { cal } 2}^{2}= & N_{F}^{2}+N_{D}^{2}+N_{B}^{2} \\
& +N_{D}^{2}+N_{B}^{2}\end{aligned}\end{array}$ \\
\hline $\begin{array}{l}\text { Master flat-field frame with } n \text { out of } \\
\text { the ten } F_{\text {cal } 1} \\
\begin{aligned} & F_{\text {cal } 1, \text { master }(n)}=\frac{1}{n} \cdot \sum_{i=1}^{n} F_{\text {cal } 1} \\
& N_{F, \text { cal } 1, \text { master }(n)}^{2}= \frac{N_{F}^{2}}{n}+\frac{N_{D}^{2}}{n}+\frac{N_{B}^{2}}{n} \\
&+\frac{N_{D}^{2}}{10}+\frac{N_{B}^{2}}{10}\end{aligned}\end{array}$ & $\begin{array}{l}\text { Master flat-field frame with } n \text { out of } \\
\text { the ten } F_{\text {cal } 2} \\
\begin{array}{c}F_{\text {cal2,master }(n)}=\frac{1}{n} \cdot \sum_{i=1}^{n} F_{\text {cal } 2} \\
N_{F, \text { cal } 2, \text { master }(n)}^{2}=\frac{N_{F}^{2}}{n}+\frac{N_{D}^{2}}{n}+\frac{N_{B}^{2}}{n} \\
+\frac{N_{D}^{2}}{n}+\frac{N_{B}^{2}}{n}\end{array}\end{array}$ \\
\hline
\end{tabular}

Table 3: Summary of two ways to generate a master flat-field frame 


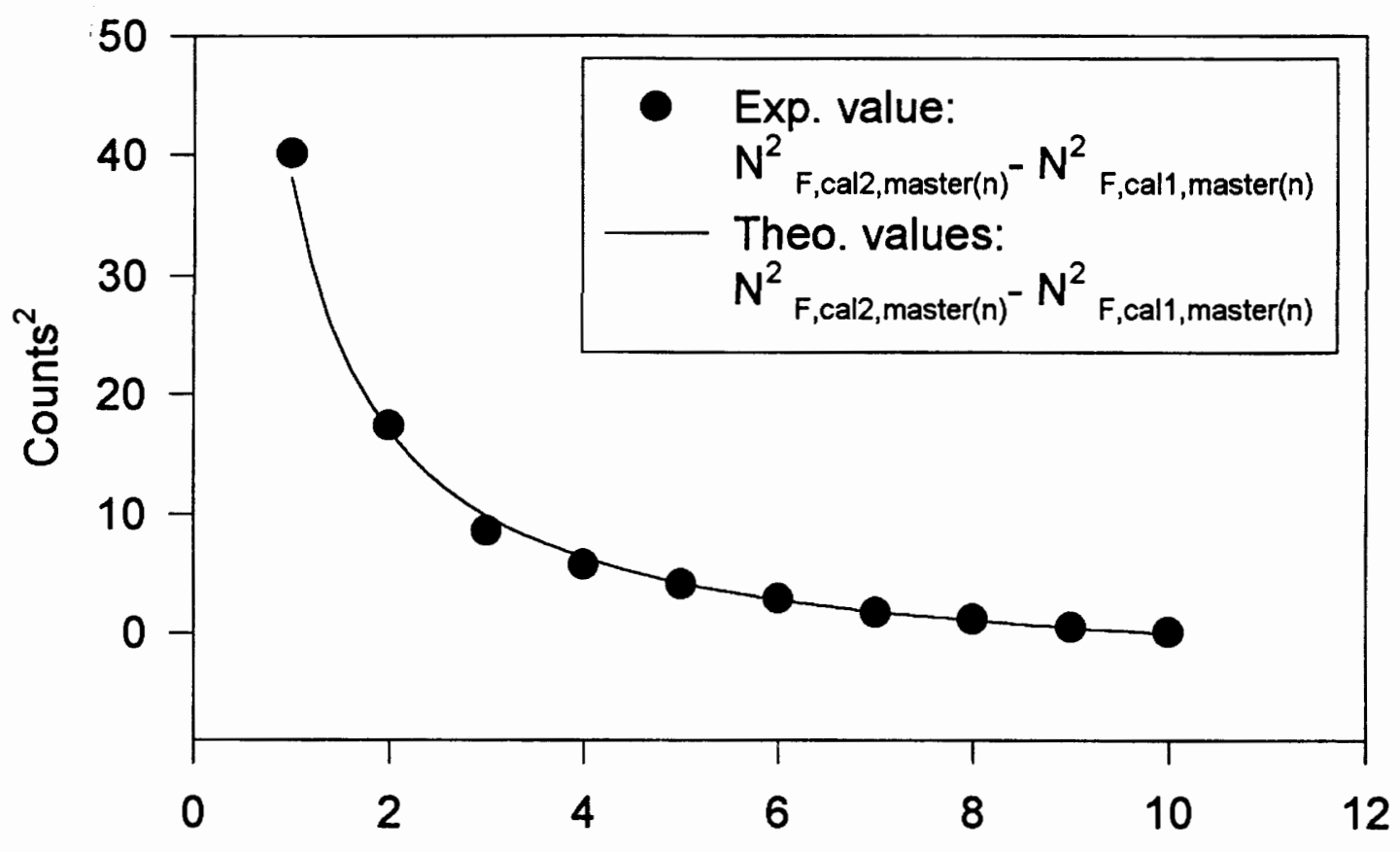

Number of frames $(n)$ used for the master flat-field frame

Figure 25: The difference of the squared noise of the two master flat-field frame calibration methods is shown. All frames are taken at $5^{\circ} \mathrm{C}$. The exposure times are 5 seconds for all dark frames and flat-field frames. 


\section{Chapter 8}

\section{Real World Example}

Considering the different ways to calibrate an image, how might a night session look like? Let us take a look at three possible night sessions and compare the advantages and disadvantages. In Table 4 an overview of the different sessions is shown with their associated 'costs'. These 'costs' are measured in terms of op. \#: The number of operations performed.

time units: The number of time units needed. Any image taken with an exposure time larger than 0 seconds counts as one time unit. A bias frame is considered to require no time to be acquired.

memory units: One saved image counts as one memory unit.

The three sessions can be described as follows. 
Session one: At the beginning of the night, a flat-field frame and a corresponding dark frame are taken. The flat-field frame is calibrated with the dark frame and normalized to an average pixel value of 1 count. During the night, images are taken with the corresponding dark frames, which are used for calibrating the images.

Session two: At the beginning of the night, 10 sets of (flat frame, dark frame), denoted as Pool-1, and 10 sets of (dark frame, bias frame), denoted as Pool-2, are taken. A master flat-field frame combining 10 flat-field frames is generated with the frames of Pool-1 and normalized to an average pixel value of 1 count. The master bias frame and the master dark frame are generated with the frames of Pool-2, each combining 10 frames. During the night, images and corresponding bias frames are taken. These bias frames are used to subtract the correct bias offset from the images. The master bias frame and the master dark frame are subtracted from the image and then the image is divided by the master flat-field frame.

Session three: In this session, a high performance CCD is used. Therefore the bias count does not drift and the bias count does not have to be divided into the bias offset and the bias structure. The session is very similar to session two, 
except there is no need to take the bias frame corresponding to each image, since it is not necessary to separately take the bias offset into account.

If we combine Equation (28), (29), (30) and (31), we can estimate the calibrated final signal with its noise level.

$$
\begin{aligned}
S_{c a l} & =\frac{S_{r a w}-\hat{D}-\hat{B}}{\hat{F}} \\
N_{c a l}^{2} & =\frac{N_{S, \text { raw }}^{2}+N_{\hat{D}}^{2}+N_{\hat{B}}^{2}+\frac{S_{c a l}^{2}}{\hat{F}^{2}} \cdot N_{\hat{F}}^{2}}{\hat{F}^{2}}
\end{aligned}
$$

The three different sessions have different $N_{\hat{D}}^{2}, N_{\hat{B}}^{2}$ and $N_{\hat{F}}^{2}$, therefore it is essential for the comparison of the noise levels to express these terms in the same 'units'. The noise in a bias count, dark count and flat field signal corresponding to a single frame, denoted as $N_{D}, N_{B}$ and $N_{F}$, respectively, are chosen as the units.

If we substitute the noise in the different calibration frames with the units $N_{D}$, $N_{B}$ and $N_{F}$, we can calculate the noise levels, shown in Table 5 , of the sessions. The noise levels in session two and three are equal due to the fact that the bias offset is noise free. Note that we find the same cancellation of the bias noise terms in session two for the same reason found in the flat-field calibration (see section 7.3, Equation (61)) 


\begin{tabular}{|c|c|c|c|c|c|c|}
\hline & Operation type & & & $\begin{array}{l}\text { op. } \\
\text { \# }\end{array}$ & $\begin{array}{l}\text { time } \\
\text { units }\end{array}$ & $\begin{array}{c}\text { memory } \\
\text { units }\end{array}$ \\
\hline \multirow[t]{4}{*}{ Session 1} & \multirow{4}{*}{$\begin{array}{l}1 \text { set (Image,Dark) } \\
\text { Image }_{\text {raw }}-\text { Dark }_{r a w} \\
\text { Image }_{D B c a l} / \text { Flat }_{c a l, n r}\end{array}$} & \multirow[t]{2}{*}{$\Rightarrow$} & \multirow{2}{*}{ Flat $_{c a l, n r m}$} & 2 & 2 & $\begin{array}{l}2 \\
2\end{array}$ \\
\hline & & & & 3 & 4 & 7 \\
\hline & & \multirow[t]{2}{*}{$\begin{array}{l}\Rightarrow \\
\Rightarrow\end{array}$} & \multirow[t]{2}{*}{$\begin{array}{l}\text { Image }_{D B c a l} \\
\text { Image }_{D B F c a l}\end{array}$} & $\begin{array}{l}1 \\
1\end{array}$ & 2 & $\begin{array}{l}2 \\
1 \\
1\end{array}$ \\
\hline & & & & 2 & 2 & 4 \\
\hline \multirow[t]{4}{*}{ Session 2} & \multirow[t]{2}{*}{$\begin{array}{l}10 \text { sets (Flat,Dark) } \\
\text { Flat }_{\text {raw }}-\text { Dark }_{\text {raw }} \\
\frac{1}{\bar{F}} \cdot\left(\frac{1}{10} \sum^{10} \text { Flat }_{c a l}\right) \\
10 \text { sets (Dark,Bias) } \\
\text { Dark }_{\text {raw }}-\text { Bias }_{\text {raw }} \\
\frac{1}{10} \sum^{10} \text { Dark }_{\text {cal }} \\
\text { Bias }_{\text {raw }}-\text { Bias }_{\text {Offset }} \\
\frac{1}{10} \sum^{10} \text { Bias }_{\text {struc }}\end{array}$} & \multirow[t]{2}{*}{$\begin{array}{l}\Rightarrow \\
\Rightarrow \\
\Rightarrow \\
\Rightarrow\end{array}$} & $\begin{array}{l}\text { Flat }_{c a l} \\
\text { Flat }_{\text {master,nrm }}\end{array}$ & $\begin{array}{c}10 \\
1 \\
10 \\
1\end{array}$ & 20 & $\begin{array}{c}20 \\
10 \\
2 \\
20 \\
10 \\
1 \\
10 \\
1\end{array}$ \\
\hline & & & $\begin{array}{l}\text { Dark }_{\text {cal }} \\
\text { Dark }_{\text {master }} \\
\text { Bias }_{\text {struc }} \\
\text { Bias }_{\text {master }}\end{array}$ & 33 & 30 & 73 \\
\hline & \multirow[t]{2}{*}{$\begin{array}{l}1 \text { set }(\text { Image,Bias) } \\
\text { Image }_{\text {raw }}-\text { Dark }_{\text {master }} \\
\text { Image }_{\text {Dal }}-\text { Bias }_{\text {master }} \\
\text { Image }_{D B c a l}-\text { Biasoffset }_{\text {sial }, \text { rm }}\end{array}$} & \multirow[t]{2}{*}{$\begin{array}{l}\Rightarrow \\
\Rightarrow \\
\Rightarrow \\
\Rightarrow\end{array}$} & \multirow[t]{2}{*}{$\begin{array}{l}\text { Image }_{D c a l} \\
\text { Image }_{D B c a l} \\
\text { Image }_{D B O c a l} \\
\text { Image }_{D B O F c a l}\end{array}$} & $\begin{array}{l}1 \\
1 \\
1 \\
1\end{array}$ & 1 & $\begin{array}{l}2 \\
1 \\
1 \\
1 \\
1\end{array}$ \\
\hline & & & & 4 & 1 & 6 \\
\hline \multirow[t]{4}{*}{ Session 3} & \multirow[t]{2}{*}{$\begin{array}{l}10 \text { sets (Flat,Dark) } \\
\text { Flat }{ }_{\text {raw }}-\text { Dark }_{\text {raw }} \\
\frac{1}{F} \cdot\left(\frac{1}{10} \sum^{10} \text { Flat }_{c a l}\right) \\
10 \text { sets (Dark,Bias) } \\
\text { Dark }_{\text {raw }}-\text { Bias }_{\text {raw }} \\
\frac{1}{10} \sum^{10} \text { Dark }_{\text {cal }} \\
\frac{1}{10} \sum^{10} \text { Bias }_{\text {raw }}\end{array}$} & $\begin{array}{l}\Rightarrow \\
\Rightarrow\end{array}$ & $\begin{array}{l}\text { Flat }_{c a l} \\
\text { Flat }_{\text {master,nrm }}\end{array}$ & $\begin{array}{c}10 \\
2 \\
\\
10 \\
1 \\
1 \\
\end{array}$ & 20 & $\begin{array}{c}20 \\
10 \\
2 \\
20 \\
10 \\
1 \\
1 \\
\end{array}$ \\
\hline & & $\begin{array}{l}\Rightarrow \\
\Rightarrow \\
\Rightarrow\end{array}$ & & 23 & 30 & 63 \\
\hline & $\begin{array}{l}1 \text { Image } \\
\text { Image }_{\text {raw }}-\text { Dark }_{\text {master }} \\
\text { Image }_{\text {Dal }}-\text { Bias }_{\text {master }} \\
\text { Image }_{D B c a l} / \text { Flat }_{\text {master,nrm }}\end{array}$ & $\begin{array}{l}\Rightarrow \\
\Rightarrow \\
\Rightarrow\end{array}$ & $\begin{array}{l}\text { Image }_{D c a l} \\
\text { Image }_{D B c a l} \\
\text { Image }_{D B F c a l}\end{array}$ & $\begin{array}{l}1 \\
1 \\
1 \\
\end{array}$ & 1 & $\begin{array}{l}1 \\
1 \\
1 \\
1\end{array}$ \\
\hline & & & & 3 & 1 & 4 \\
\hline
\end{tabular}

Table 4: Three possibilities of how a night session might look are shown. 


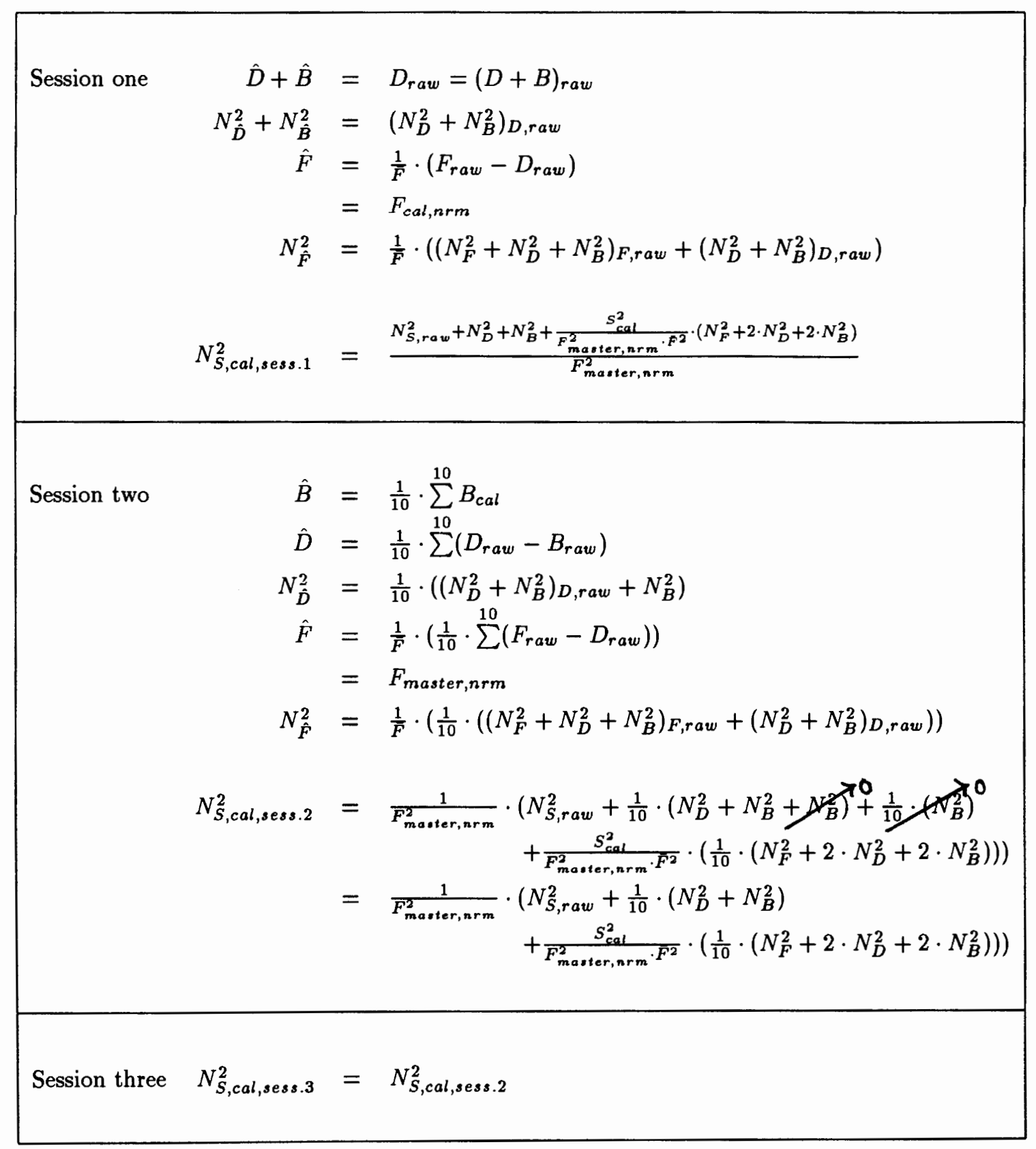

Table 5: This Table shows the noise levels of the night sessions. Note that the noise level of session three is equal to the noise level of session two 


\subsection{Comparison of the Three Night Sessions}

Following is the comparison of the night sessions.

Comparison of session one and session two: In Table 4 it can be seen that session one is superior in processing speed and memory requirements. The straightforward processing makes a correct calibration easy to perform. A disadvantage is that the noise level is higher compared to session two. Another disadvantage is that the dark exposure time has to be equal to the image exposure time, since the dark frame includes the bias count. This makes it impossible to scale the dark frame to another exposure time.

In session two, it is possible to use the same calibration frames the whole night. After the calibration frames are obtained at the beginning of the night, only the images with the corresponding bias frames have to be taken during the night. This is a big advantage for long night sessions since a bias frame has zero exposure time and therefore only half of the imaging time is needed compared to session one.

Comparison of session two and session three: Since session three is very similar to session two, it has the same advantages and disadvantages, but session three is a little faster than session two. Furthermore, it is no longer necessary to take a bias frame corresponding to the image, therefore this session is not 
as memory intensive. The major disadvantage is that session three can only be performed if the CCD camera used is a high performance camera, since it is essential for this calibration procedure that the bias count does not float.

\subsection{Quantitative Estimation of the Noise Levels}

The raw signal $\left(S_{\text {raw }}\right)$ can functionally be split into a real signal $\left(S_{\text {real }}\right)$, a dark count $(D)$ and a bias count $(B)$

$$
\begin{aligned}
S_{\text {raw }} & =S_{\text {real }}+D+B \\
N_{S, \text { raw }}^{2} & =N_{S, \text { real }}^{2}+N_{D}^{2}+N_{B}^{2}
\end{aligned}
$$

Using this we can describe the noise levels in the three night sessions as follows (see Table 5):

$$
\begin{aligned}
& N_{S, \text { cal }, \text { session } 1}^{2}=\frac{1}{F_{m a s t e r, n r m}^{2}} \cdot\left(\left(N_{S, \text { real }}^{2}+N_{D}^{2}+N_{B}^{2}\right)_{S, \text { raw }}+N_{D}^{2}+N_{B}^{2}+\right. \\
& \left.\frac{S_{c a l}^{2}}{\bar{F}_{\text {mater, }, n r m} \cdot \bar{F}^{2}} \cdot\left(N_{F}^{2}+2 \cdot N_{D}^{2}+2 \cdot N_{B}^{2}\right)\right) \\
& N_{S, \text { cal }, \text { session } 2}^{2}=\frac{1}{F_{\text {master, } n r m}^{2}} \cdot\left(\left(N_{S, \text { real }}^{2}+N_{D}^{2}+N_{B}^{2}\right)_{S, \text { raw }}+\frac{1}{10} \cdot\left(N_{D}^{2}+N_{B}^{2}\right)+\right. \\
& \frac{S_{c a l}^{2}}{F_{\text {mater, nrm }} \cdot \bar{F}^{2}} \cdot\left(\frac{1}{10} \cdot\left(N_{F}^{2}+2 \cdot N_{D}^{2}+2 \cdot N_{B}^{2}\right)\right)
\end{aligned}
$$


A quantative estimate of the noise in the final image can be done if values are found for $N_{B}, N_{D}, N_{F}$ and $N_{S, \text { real }}$. This is easy for $N_{D}, N_{F}$ and $N_{S, \text { real }}$, since these noises correspond to a Poisson count. Then $N_{D}^{2}, N_{F}^{2}$ and $N_{S, \text { real }}^{2}$ can be estimated with the dark count $D$, the flat-field count $F$ and the real signal $S_{\text {real }}$, respectively. The estimate for the bias noise has to be done differently since the bias count is not a Poisson count. An easy way to obtain an estimate of the average noise in the bias count is to use the 'subtraction method' described in section 3.2.1. The bias noise can be estimated by simply calculating the average noise in a difference frame, generated by subtracting two bias frames from each other, and then using the following equation:

$$
N_{B}^{2}=\frac{N_{d i f f}^{2}}{2}
$$

The average bias noise corresponding to one bias frame is denoted with $\bar{N}_{B}$. The normalized flat-field count $F_{\text {master,nrm }}$ can be estimated with 1 count (see chapter 6 ). With correct calibration, the real signal $S_{\text {real }}$ should be very close to the calibrated signal $S_{c a l}$. Using all these estimates, we can approximate the noise of the final image with

$$
N_{S, \text { cal }, \text { session } 1}^{2} \approx S_{c a l}+D+\bar{N}_{B}^{2}+D+\bar{N}_{B}^{2}+\frac{S_{c a l}^{2}}{\bar{F}^{2}} \cdot\left(F+2 \cdot D+2 \cdot \bar{N}_{B}^{2}\right)
$$




$$
N_{S, \text { cal,session } 2}^{2} \approx S_{c a l}+D+\bar{N}_{B}^{2}+\frac{1}{10} \cdot\left(D+\bar{N}_{B}^{2}\right)+\frac{S_{c a l}^{2}}{\bar{F}^{2}} \cdot\left(\frac{1}{10} \cdot\left(F+2 \cdot D+2 \cdot \bar{N}_{B}^{2}\right)\right)
$$

This approximation can be applied to estimate the average noise in the image using averaged $D, \bar{N}_{B}, F$ and $S_{c a l}$ or to estimate the noise in a specific pixel using the counts of this specific pixel.

To calculate this lengthy term is very time consuming but, in most cases it is possible to get a rough estimate by simplifying the Equation (71) or Equation (72) to just the leading terms. The following sections detail this process for several different cases.

\section{Faint objects}

Suppose the average flat-field signal $(\bar{F})$ is much bigger, say $x$ times bigger, than the real signal $\left(S_{\text {real }}\right)$, then we can write

$$
\frac{S_{r e a l}^{2}}{\bar{F}^{2}}=\frac{1}{x^{2}}
$$

If we plug this in Equation (71), we get

$$
N_{S, c a l, s e s s i o n 1}^{2} \approx S_{c a l}+2 \cdot D+2 \cdot \bar{N}_{B}^{2}+\frac{1}{x^{2}} \cdot\left(F+2 \cdot D+2 \cdot \bar{N}_{B}^{2}\right)
$$

and since

$$
\frac{1}{x^{2}} \cdot\left(F+2 \cdot D+2 \cdot \bar{N}_{B}^{2}\right) \leq \frac{1}{x} \cdot\left(S_{c a l}+2 \cdot D+2 \cdot \bar{N}_{B}^{2}\right)
$$


the total noise can be estimated by

$$
N_{S, c a l, \text { session } 1}^{2} \leq S_{c a l}+2 \cdot D+2 \cdot \bar{N}_{B}^{2}+\frac{1}{x} \cdot\left(S_{c a l}+2 \cdot D+2 \cdot \bar{N}_{B}^{2}\right)
$$

For a sufficiently large value of $x$, the second term cancels out and we can further simplify to

$$
N_{S, c a l, \text { session } 1}^{2} \approx S_{\text {cal }}+2 \cdot D+2 \cdot \bar{N}_{B}^{2}
$$

\section{Very bright objects}

The exposure time for bright objects is short and therefore also the accumulated dark count is very small compared to the real signal $\left(S_{\text {real }}\right)$, even for very hot pixels. This can be applied to the flat-field count $(F)$, if the flat-field count is sufficient high. In this case, the contribution of the dark count and the bias count can be neglected and Equation (71) can be simplified to

$$
N_{S, \text { cal }, \text { session } 1}^{2} \approx S_{c a l}+\frac{S_{c a l}^{2}}{\bar{F}^{2}} \cdot F
$$

Figure 2 shows a faint object, the Ring Nebula M-57. The white specks caused by the dark count can clearly be seen in the uncalibrated image. In Figure 3, a bright object, the Hercules Cluster M-13, is shown. The dark count can hardly be seen, even in the uncalibrated image. Obviously, dark subtraction plays a larger role for faint objects. The reason being a larger exposure time for faint objects ${ }^{1}$ as well as a smaller ratio of the real signal to the dark count.

\footnotetext{
${ }^{1}$ More dark count is accumulated.
} 


\section{Combining celestial images}

In order to obtain a better signal-to-noise ratio, it is advantageous to combine several 'equal' images of the same object, i.e., the images should have the same signal and noise levels and the object should be on the same spot in the image. Say $n$ images are combined to a master image $\left(S_{\text {cal,master }(n)}\right)$. If the images are calibrated as described in session one, the noise $\left(N_{S, c a l, m a s t e r(n), \text { session1 } 1}\right)$ decreases with $\sqrt{n}$ :

$$
\begin{aligned}
S_{c a l, \text { master }(n), \text { session } 1} & =\frac{1}{n} \cdot \sum_{i=1}^{10} S_{i, c a l, \text { session } 1} \\
N_{S, \text { cal,master }(n), \text { session } 1}^{2} & =\frac{N_{S, c a l, \text { session } 1}^{2}}{n}
\end{aligned}
$$

It is different if the images are calibrated as described in session two, since the same calibration frames, i.e., the same master bias frame, master dark frame and master flat field frame, were used to calibrate the images. Then, the noise caused by subtracting the master dark frame and the master bias frame and the noise caused by dividing through the master flat-field frame does not decrease by combining the calibrated object images.

$$
\begin{aligned}
N_{S, \text { cal }, \text { session } 2}^{2}=\frac{1}{F_{\text {master, nrm }}^{2}} \cdot & \left(\left(N_{S, \text { real }}^{2}+N_{D}^{2}+N_{B}^{2}\right)+\frac{1}{10} \cdot\left(N_{D}^{2}+N_{B}^{2}\right)+\right. \\
& \frac{S_{c a l}^{2}}{F_{\text {mater,nrm }}^{2} \cdot \bar{F}^{2}} \cdot\left(\frac{1}{10} \cdot\left(N_{F}^{2}+2 \cdot N_{D}^{2}+2 \cdot N_{B}^{2}\right)\right)
\end{aligned}
$$




$$
\begin{aligned}
& N_{S, \text { cal,master }(n), \text { session } 2}^{2}= \\
& \frac{1}{F_{\text {master }, n r m}^{2}} \cdot \quad\left(\left(\frac{N_{S, \text { real }}^{2}+N_{D}^{2}+N_{B}^{2}}{n}\right)+\frac{1}{10} \cdot\left(N_{D}^{2}+N_{B}^{2}\right)+\right. \\
& \frac{S_{c a l}^{2}}{F_{\text {master,nrm }}^{2} \cdot \tilde{F}^{2}} \cdot\left(\frac{1}{10} \cdot\left(N_{F}^{2}+2 \cdot N_{D}^{2}+2 \cdot N_{B}^{2}\right)\right)
\end{aligned}
$$

This can have a major influence on the noise estimate. Suppose the noise in the raw signal is $m$ times higher than the other noise caused by subtracting the dark count, the bias count and by dividing by the flat-field count.

$$
N_{S, \text { cal }, \text { session } 2}^{2}=N_{S, \text { raw }}^{2}+\frac{1}{m} \cdot N_{S, \text { raw }}^{2}
$$

The second term can be neglected for sufficiently large $m$, for reasons mentioned earlier, and the main contribution to the total noise is the noise in the raw signal.

$$
N_{S, \text { cal }, \text { session } 2}^{2} \approx N_{S, \text { raw }}^{2}
$$

It is very attractive to use the noise of the raw signal as the estimate of the noise of the combined images.

$$
N_{S, \text { cal,master }(n), \text { session } 2}^{2} \approx \frac{N_{S, r a w}^{2}}{n}
$$

But this is meaningful only to a limit. Say $m=n$, then we can use Equation (85) to calculate the noise in the master image, shown below: 


$$
\begin{aligned}
N_{S, \text { cal,session } 2}^{2} & =N_{S, \text { raw }}^{2}+\frac{1}{n} \cdot N_{S, \text { raw }}^{2} \\
N_{S, \text { cal,master(n),session } 2}^{2} & =\frac{1}{n} \cdot N_{S, \text { raw }}^{2}+\frac{1}{n} \cdot N_{S, \text { raw }}^{2} \\
& =2 \cdot \frac{N_{S, \text { raw }}^{2}}{n}
\end{aligned}
$$

Obviously, the noise calculated with Equation (89) is larger by a factor of two compared to the noise calculated with Equation (87). It is important that the noise, which could be neglected for one image, now plays a more important part in the total noise of the master image. 


\section{Chapter 9}

\section{Conclusions}

The dependence of the bias count, caused by charging the chip, on the temperature was examined in several experiments. It was shown that the bias count and the corresponding bias noise decreased slightly if the CCD-camera is cooled down. For temperatures lower than $5^{\circ} \mathrm{C}$, the bias offset and the bias structure stayed nearly unchanged. But more importantly, the bias offset and consequently the bias count were much more stable at low temperatures and therefore, it was possible to subtract the bias count with greater accuracy at low temperatures.

Random motions of electrons within the chip are the sources of dark count. The dark count showed a linear dependence on the exposure time, even though different pixels have different dark counts. This made it possible to use dark frames with different dark exposure times by scaling them with a constant. As expected, multiple 
pixel populations, each with a well defined average dark count, could be observed. $95 \%$ of the pixels were in the main population, $2 \%$ in the hot pixel population one and $0.1 \%$ in the hot pixel population two. The remaining $3 \%$ of the population were uniformly distributed.

The dark count showed a well-defined exponential temperature dependence. The measured doubling temperature $\Delta T$ was $5.71^{\circ} \mathrm{C}$ for the chip used. It turned out that the hot pixels have a higher doubling temperature. For the first hot pixel population, a doubling temperature $\Delta T_{\text {hot }}$ of $7.40^{\circ} \mathrm{C}$ was found.

It was shown that it could be an advantage to use dark frames scaled down from a high temperature for dark subtraction, due to the fact that a dark frame taken at a higher temperature with the same exposure time has a larger signal-to-noise ratio. It was found that in practice, dark subtraction with a dark frame taken at another temperature led to a wrong result; this is caused by the fact that the hot pixels have a different doubling temperature than the main population. Although the main population can be scaled down very accurately, the disadvantage of incorrect scaled-down hot pixels overshadows the better signal-to-noise ratio, even though the hot pixels are only $5 \%$ of the total population.

An unexpected effect was observed when flat-field frames or other light frames were taken before dark frames or bias frames: The average count increased. For a temperature of $-5^{\circ} \mathrm{C}$ the dark count increased by a factor of 15 if a flat-field frame 
was taken before with a sufficiently long exposure time. Even for non-saturated flatfield frames with short exposure times, the average dark count of a dark frame taken directly after the flat field frame increased by a factor of 3 . It was first thought that the incoming light caused a temperature increase on the surface of the chip which could result in a multiplicative increase of dark count. This, however, was shown not to be the case, since the hot pixels did not increase by this factor and only an offset, denoted as dark offset, was observed. From these observations, it was surmised that the effect was due to storage effects. The halftime of the dark offset was 50.5 seconds \pm 1.7 seconds. The dark offset is a false signal added to the dark count and missing from the light count. The ratio of two pixels with different light counts deteriorates by this dark offset, since the storage is not linearly dependent on the light count. In order to remove the error caused by adding the dark offset to the dark count, dark frames should be taken before light frames are taken.

It has been shown that the generation of a master frame does not always decrease the noise as expected. The noise decreases with $\sqrt{n}(n=$ number of frames used) if independent frames are used; but if in the calibration process the same frame is used, the noise of the master image will not decrease as fast. This fact is overlooked in the literature. For generating master dark frames, it is sufficient to subtract just raw bias frames instead of both master bias frames and bias offset for the bias count subtraction. Similarly, in master flat-field frame generation, the subtraction 
of just raw dark frames instead of both master bias frames and master dark frames leads to the same noise level. In celestial images, the use of master frames increases the signal-to-noise ratio. On a case by case basis, one might need to decide if the advantages of a higher signal-to-noise ratio is worth the additional processing time and increased memory requirements. For long night sessions it can be advantageous to spend time at the beginning of the night to generate master calibration frames since it is then no longer necessary to take additional frames for calibration after each image, due to the fact that one can use the same master dark frame for all dark count calibrations.

From the result of the night sessions described above, it was shown how to estimate the final noise in a calibrated image. Starting from the noise in the calibrated master flat field frame, the total noise was calculated. Several examples were given onhow to simplify the estimate for real applications like faint stars and bright objects. The possibility to easily process a noise map of acalibrated image using the image arithmetic features in the software usedwas shown. 


\section{Appendix}

\section{Problems with the software}

\section{MIRA}

The software described in section 3.1.2 was used for all image processing and for controlling the CCD camera. Several problems with the software arose during the research done.

\section{A.1 Median Combining}

The combining feature in the software was extensively used. It was recognized, that the median combining led to unexpected results. Instead of improving the noise level compared to mean combining, it increased the noise level. Furthermore, the 
noise showed different noise levels for odd and even numbers of frames used for the master frame. The 'Saw Tooth'-like curve can be seen in Figure 26.

Out of 3 pools, each with 10 bias frames taken at $-10^{\circ} \mathrm{C}$, the master frames were processed. The noise in the frames was measured using the subtraction method described in section 3.2.1.

$$
N_{B, \operatorname{master}(n), \exp }^{2}=\frac{N_{D i f f}^{2}}{2}
$$

where $N_{B, \operatorname{master(n)}}$ is the noise in the master bias frame and $N_{D i f f}$ is the noise in the difference frame.

In Figure 26, the measured noises of median and mean combined master bias frames (see Equation (90)) are shown. For comparison, the theoretical noise for a master bias frame is calculated using

$$
N_{B, \text { master }(n), \text { theo }}^{2}=\frac{N_{B, e x p}^{2}}{n}
$$

where $N_{B, \text { master(n),theo }}$ is the theoretical noise in the master bias frame and $N_{B, \exp }$ is the noise in a single bias frame measured with the subtraction method.

Obviously, the noises in the mean combined master bias frames are very close to the predicted theoretical values (see Figure 26). The noises in the median combined master bias frames have oddball values, that do not agree with the theoretical values. It can be concluded that there is an error in the median combining feature. 


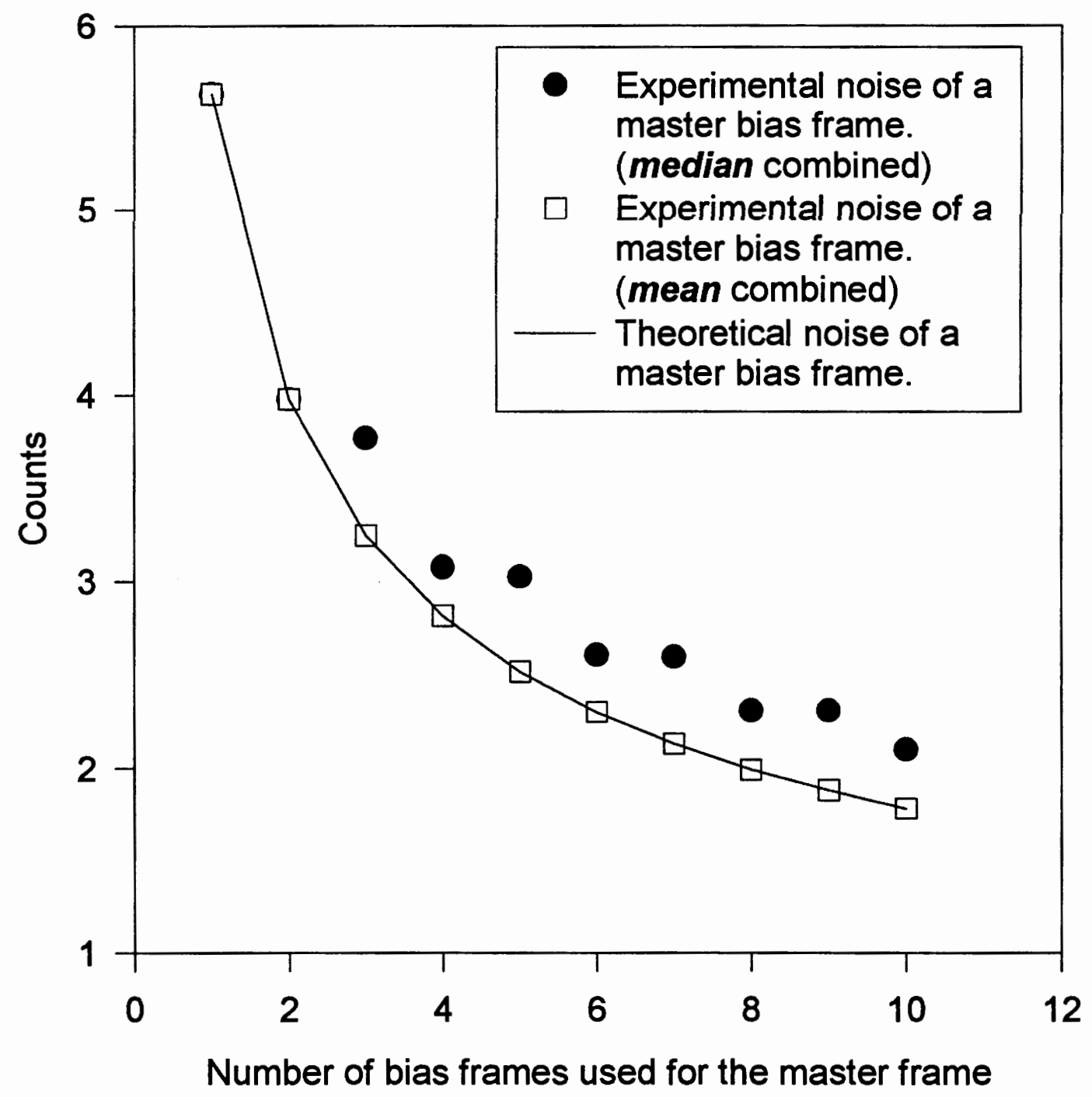

Figure 26: Comparison of the noise in a median or mean combined master bias frame. The bias frames are taken at $-10^{\circ} \mathrm{C}$ 


\section{A.2 Normalization in Combining feature}

The normalization in the combining feature causes problems, too. If we normalize with median as reference statistic, the median is calculated wrong and therefore the scaling constant is calculated wrong. A demo of this defective calculation is as follows: A pool of 15 dark frames ${ }^{1}$ were taken and calibrated by subtracting the corresponding bias frames. Using the 'image arithmetic' feature to calculate the median of the whole frame gives the expected values, median dark count around 20 counts. But using the normalization feature in the combining images feature gives a completely different result. The first value is correct, but the following medians have an increasing error and therefore a wrong scaling constant. The error converges in this example to a value of -13 . Since most of the frames are now scaled down to a value of $20-13=7$, the resulting combined image has a median of 8 instead of the correct value of around 20 . The corresponding sections of the Mira logbook are:

$\begin{array}{llllll}\text { file } & \text { mean } & \text { median } & \text { sdev } & \text { min } & \max \\ \text { DARKC12.001 } & 32.22268 & 20 & 73.734106 & -446 & 10065 \\ \text { DARKC12.002 } & 31.864064 & 20 & 73.43877 & -458 & 10229 \\ \text { DARKC12.003 } & 31.149502 & 20 & 72.085515 & -432 & 10032 \\ \text { DARKC12.004 } & 30.406759 & 19 & 70.895907 & -440 & 9871\end{array}$

\footnotetext{
${ }^{1}$ Exposure time: 30 seconds; Temperature: $12^{\circ} \mathrm{C}$
} 


\begin{tabular}{|c|c|c|c|c|c|}
\hline DARKC12.005 & 30.196883 & 19 & 70.622178 & -429 & 9907 \\
\hline DARKC12.006 & 30.113085 & 19 & 70.544217 & -426 & 9856 \\
\hline DARKC12.007 & 30.686472 & 19 & 71.71651 & -451 & 10057 \\
\hline DARKC12.008 & 31.259196 & 20 & 72.722375 & -439 & 10172 \\
\hline DARKC12.009 & 31.811946 & 20 & 73.459893 & -453 & 10218 \\
\hline DARKC12.010 & 32.159251 & 20 & 74.014917 & -455 & 10223 \\
\hline DARKC12.011 & 32.663443 & 21 & 74.940597 & -476 & 10402 \\
\hline DARKC12.012 & 33.416983 & 21 & 75.736816 & -463 & 10411 \\
\hline DARKC12.013 & 33.953113 & 22 & 76.558599 & -455 & 10544 \\
\hline DARKC12.014 & 33.545058 & 21 & 75.814116 & -482 & 10423 \\
\hline DARKC12.015 & 32.452094 & 20 & 74.249486 & -461 & 10242 \\
\hline image file & ref. median & constant & & & \\
\hline DARKC12.001 & 20 & 0 & & & \\
\hline DARKC12.002 & 27 & -7 & & & \\
\hline DARKC12.003 & 29 & -9 & & & \\
\hline DARKC12.004 & 31 & -11 & & & \\
\hline DARKC12.005 & 32 & -12 & & & \\
\hline DARKC12.006 & 32 & -12 & & & \\
\hline DARKC12.007 & 32 & -12 & & & \\
\hline DARKC12.008 & 33 & -13 & & & \\
\hline
\end{tabular}




\begin{tabular}{|c|c|c|c|c|c|}
\hline DARKC12.009 & 33 & -13 & & & \\
\hline DARKC12.010 & 33 & -13 & & & \\
\hline DARKC12.011 & 33 & -13 & & & \\
\hline DARKC12.012 & 33 & -13 & & & \\
\hline DARKC12.013 & 33 & -13 & & & \\
\hline DARKC12.014 & 33 & -13 & & & \\
\hline DARKC12.015 & 33 & -13 & & & \\
\hline \multicolumn{6}{|c|}{ median of 15 images $\rightarrow$ DUMMY.MAS } \\
\hline file & mean & median & sdev & $\min$ & $\max$ \\
\hline DUMMY . MAS & 20.564695 & 8 & 72.826975 & -465 & 10205 \\
\hline image file & ref. median & constant & & & \\
\hline
\end{tabular}

Furthermore, a bias frame was used as reference frame instead of the correct dark frame. The calculated medians of the dark frames are very close to the median of the reference bias frame (around 2242 counts) instead of having the correct value of 20 counts. The corresponding section of the Mira logbook is:

image file ref. median constant

DARKC12.001 $2242 \quad 0$ 


\begin{tabular}{|c|c|c|c|c|c|}
\hline DARKC12.002 & 2245 & -3 & & & \\
\hline DARKC12.003 & 2246 & -4 & & & \\
\hline DARKC12.004 & 2247 & -5 & & & \\
\hline DARKC12.005 & 2247 & -5 & & & \\
\hline DARKC12.006 & 2247 & -5 & & & \\
\hline DARKC12.007 & 2248 & -6 & & & \\
\hline DARKC12.008 & 2248 & -6 & & & \\
\hline DARKC12.009 & 2248 & -6 & & & \\
\hline DARKC12.010 & 2248 & -6 & & & \\
\hline DARKC12.011 & 2248 & -6 & & & \\
\hline DARKC12.012 & 2248 & -6 & & & \\
\hline DARKC12.013 & 2248 & -6 & & & \\
\hline DARKC12.014 & 2248 & -6 & & & \\
\hline DARKC12.015 & 2248 & -6 & & & \\
\hline \multicolumn{6}{|c|}{ median of 15 images $\rightarrow$ DUMMY.MAS } \\
\hline file & mean & median & sdev & $\min$ & $\max$ \\
\hline DUMMY .MAS & 26.768787 & 14 & 72.804781 & -459 & 10212 \\
\hline
\end{tabular}




\section{A.3 Minor Problems}

The following are some minor problems found with the software:

- In the 'File Manager', it is not possible to copy an image from outside the main directory: Instead of copying the header file and the generic data file, Mira is copying two generic data files.

- The text editor has problems with the last line:

- If the last line is empty, the text editor deletes two lines instead of one line.

- If the last line is not empty, deleting the last line causes the computer to freeze.

- The feature 'Image \& Image' (and other similar features) do not recognize the last line of a file list if the file list was created with the text editor.

- It is not possible to change the keyword value of the keyword 'Refbias' with the 'Change Keyword Value' feature. Error message: 'The value is not consistent with the data type of the keyword'.

- 'Default Path' feature: If you use the file dialog window, you have to select a file instead of selecting only a path, and the filename is copied in the default path, too. 
- The 'Write Fts Image' feature saves the fts image always in the main directory (C: Mira) and ignores the default paths.

- Occasionally, the computer resets while using the 'Flat Field' feature.

- At the beginning of the research done, a computer with a 486 processor, a brand new hard disk and a brand new motherboard was used. After two weeks, the hard disk crashed and it was not possible to restore the data. Since there were all these problems with the 486 computer, a brand new Pentium with $1.2 \mathrm{Gbyte}$ hard disk was used instead. After 40 days of using the Mira Software exclusively, the hard disk crashed again and all data were lost. The next exchanged hard disk had increasing bad sectors in the first days, but fortunately, it stopped after one week. In both cases, when the hard disks crashed, the hard disks were full or almost full. It might be coincidence, but perhaps something went wrong when the Mira software tried to write to a full hard disk. Note that the hard disk was extensively used since around 7Gbyte data were processed. 


\section{Bibliography}

[1] L. Holst A.D. Barbour and S. Janson. Poisson Approximation. Oxford University Press, 1992.

[2] Frank C. Andrews. Thermodynamics: Principles and Applications. WileyInterscience, 1971.

[3] David Halliday and Robert Resnick. Fundamentals of Physics. John Wiley \& Sons, 1986.

[4] Glenn E. Healey and Raghava Kondepudy. Radiometric CCD camera calibration and noise estimation. IEEE Transactions on Pattern Analysis and Machine Intelligence, 16(3):267-276, 1994.

[5] William J. Kaufmann III. Universe. W.H. Freeman and Company, 1994.

[6] Jerome Kristian and Morley Blouke. Charge-coupled devices in astronomy. Scientific American, 247:67-74, 1982. 
[7] Eric J. Lerner. Charge-coupled devices capture image information. Laser Focus World, pages 103-112, August 1996.

[8] N. Hastings M. Evans and B. Peacock. Statistical Distributions. John Wiley \& Sons, Inc., 1993.

[9] Erich Meyer and Herbert Raab. CCD astrometry. CCD Astronomy, pages 12-14, Winter 1995.

[10] Michael V. Newberry. The signal to noise connection part I. CCD Astronomy, pages 13-17, Summer 1994.

[11] Michael V. Newberry. The signal to noise connection part II. CCD Astronomy, pages 12-15, Fall 1994.

[12] Michael V. Newberry. Dark frames. CCD Astronomy, pages 12-14, Summer 1995.

[13] Michael V. Newberry. Recovering the signal. CCD Astronomy, pages 18-21, Spring 1995.

[14] Michael V. Newberry. Pursuing the ideal flat field. CCD Astronomy, pages 18-21, Winter 1996.

[15] D. Keith Robinson Philip R. Bevington. Data Reduction and Error Analysis for the Physical Sciences. W.H. Freeman and Company, 1994. 
[16] Fabrizio Pinto. CCD's in the mechanics lab. The Physics Teacher, 33:436-440, 1995.

[17] F. Reif. Fundamentals of Statistical and Thermal Physics. McGraw-Hill Book Company, 1965. 